\title{
NUMERICAL ANALYSIS FOR A CHEMOTAXIS-NAVIER-STOKES SYSTEM
}

\author{
Abelardo Duarte-Rodríguez ${ }^{1}$, María Ángeles Rodríguez-Bellido ${ }^{2}$, \\ Diego A. Rueda-Gómez ${ }^{1}$ and Élder J. Villamizar-RoA ${ }^{1, *}$
}

\begin{abstract}
In this paper we develop a numerical scheme for approximating a $d$-dimensional chemotaxisNavier-Stokes system, $d=2,3$, modeling cellular swimming in incompressible fluids. This model describes the chemotaxis-fluid interaction in cases where the chemical signal is consumed with a rate proportional to the amount of organisms. We construct numerical approximations based on the Finite Element method and analyze optimal error estimates and convergence towards regular solutions. In order to construct the numerical scheme, we use a splitting technique to deal with the chemo-attraction term in the cell-density equation, leading to introduce a new variable given by the gradient of the chemical concentration. Having the equivalent model, we consider a fully discrete Finite Element approximation which is well-posed and mass-conservative. We obtain uniform estimates and analyze the convergence of the scheme. Finally, we present some numerical simulations to verify the good behavior of our scheme, as well as to check numerically the optimal error estimates proved in our theoretical analysis.
\end{abstract}

Mathematics Subject Classification. 35Q35, 92C17, 65M12, 65M15, 65M60.

Received October 21, 2019. Accepted June 4, 2020.

\section{INTRODUCTION}

Chemotaxis is the oriented movement of cells towards the concentration gradient of certain chemicals in their environment. In particular, when the movement of cells is directed towards the increasing concentration of a signal, the phenomenon is known as chemotaxis by attraction. This kind of phenomena, which play an outstanding role in a large range of biological applications, are modeled, in their simplest form, by the KellerSegel system. However, some experimental studies, as reported in $[17,30]$, reveal that the chemotactic motion in liquid environments affects substantially the migration of cells. Some examples of these facts are the phenomenon of broadcast spawning, the pattern generation and spontaneous emergence of turbulence in populations of aerobic bacteria suspended in sessile drops of water [29]. This kind of interaction can be modeled through a

\footnotetext{
Keywords and phrases. Chemotaxis-Navier-Stokes system, finite elements, convergence rates, error estimates.

1 Escuela de Matemáticas, Universidad Industrial de Santander, A.A. 678, Bucaramanga, Colombia

* Corresponding author: jvillami@uis.edu.co

2 Departamento de Ecuaciones Diferenciales y Análisis Numérico and IMUS, Universidad de Sevilla, Facultad de Matemáticas, C/ Tarfia, S/N, 41012 Sevilla, Spain
} 
chemotaxis-Navier-Stokes system. This model is given by the following system of PDE:

$$
\left\{\begin{array}{l}
\eta_{t}+\mathbf{u} \cdot \nabla \eta=D_{n} \Delta \eta-\chi \nabla \cdot(\eta \nabla c), \\
c_{t}+\mathbf{u} \cdot \nabla c=D_{c} \Delta c-\gamma \eta c \\
\rho\left(\mathbf{u}_{t}+(\mathbf{u} \cdot \nabla) \mathbf{u}\right)=D_{\mathbf{u}} \Delta \mathbf{u}-\nabla \pi+\eta \nabla \phi, \\
\nabla \cdot \mathbf{u}=0,
\end{array}\right.
$$

where $\eta=\eta(\boldsymbol{x}, t), c=c(\boldsymbol{x}, t), \pi(\boldsymbol{x}, t)$ and $\mathbf{u}(\boldsymbol{x}, t)$ denote respectively the cell density, the concentration of an attractive chemical signal, the hydrostatic pressure, and the velocity field of the fluid at position $x \in \Omega \subset \mathbb{R}^{d}$, $d=2,3$, and time $t \in(0, T]$. This model describes the interaction between a type of cells (e.g., bacteria), and a chemical signal which is consumed with a rate proportional to the amount of organisms. The cells and chemical substance are transported by a viscous incompressible fluid under the influence of a force due to the aggregation of cells. The equation for the velocity field $\mathbf{u}$ is described by the incompressible Navier-Stokes system with forcing term given by $-\eta \nabla \phi$ (where the function $\phi$ is given and depends only on $\boldsymbol{x}$ ), which represents the effects due to density variations caused by cell aggregation. The parameters $\gamma, \chi, D_{n}, D_{c}, \rho$ and $D_{\mathbf{u}}$ are positive constants that represent the consumption rate of the chemical signal, the chemotactic coefficient, the cell diffusion constant, the chemical diffusion constant, the fluid density and the viscosity of fluid, respectively.

System (1.1) is completed with the following initial and boundary data:

$$
\left\{\begin{array}{l}
{[\eta(\boldsymbol{x}, 0), \quad c(\boldsymbol{x}, 0), \quad \mathbf{u}(\boldsymbol{x}, 0)]=\left[\eta_{0}(\boldsymbol{x}), \quad c_{0}(\boldsymbol{x}), \quad \mathbf{u}_{0}(\boldsymbol{x})\right], \quad \boldsymbol{x} \in \Omega,} \\
\frac{\partial \eta(\boldsymbol{x}, t)}{\partial \boldsymbol{\nu}}=\frac{\partial c(\boldsymbol{x}, t)}{\partial \boldsymbol{\nu}}=0, \quad \mathbf{u}(\boldsymbol{x}, t)=0, \quad \boldsymbol{x} \in \partial \Omega, \quad t \in(0, T) .
\end{array}\right.
$$

The mathematical understanding of the existence and uniqueness of a solution for (1.1) and (1.2) is quite challenging, due the coupling between the Navier-Stokes equations and the chemotaxis system. However, there are several results of existence, uniqueness, regularity and qualitative properties of the solutions for system (1.1) and (1.2) and related models (see for instance, [8,18,20,31-33,35] and references therein). In [31], the existence of global classical solutions in two-dimensional bounded convex domains was proved. The results of [31] were extended to nonconvex domains in [18]. Results of convergence of classical solutions to the corresponding stationary model were analyzed in $[32,35]$. In [33], the existence of global weak solutions in bounded threedimensional convex domains was proved, considering the Stokes system instead of the Navier-Stokes equations, that is, neglecting the nonlinear term $(\mathbf{u} \cdot \nabla) \mathbf{u}$ in the fluid equation. The existence of global weak solutions for the full three-dimensional chemotaxis-Navier-Stokes model in bounded convex domains was obtained in [33]. In [33], Winkler also proved that any eventual energy solution becomes smooth after some waiting time. The existence of weak solutions for general bounded domains of $\mathbb{R}^{3}$ for the chemotaxis-Navier-Stokes system with logistic source was proved in [20]. In the same paper, Lankeit also proved that after some waiting time, the weak solutions become smooth and converge to some steady state. Results of global existence of mild solutions in bounded domains of $\mathbb{R}^{d}, d=2,3$, for small initial data in $L^{p}$-spaces were obtained in [8].

From the numerical point of view, there are only a few works focused on system (1.1) and (1.2) and related systems. For Keller-Segel system (i.e., with chemo-attraction and linear production) and assuming that there is no interaction with the fluid, we refer $[9,10,23,26,27]$. In [10], the convergence of a finite volume was analyzed; and in $[26,27]$, some error estimates for a conservative Finite Element (FE) approximation were obtained. In [9], some error estimates for a fully discrete discontinuous FE method were proved for a chemotaxis-production model. A mixed FE approximation was analyzed in [23]. In the same framework of Keller-Segel system, some previous energy stable numerical schemes have also been analyzed ( $c f$. [2]). In addition, unconditionally energy stable time-discrete numerical schemes and fully discrete FE schemes for a chemo-repulsion model with quadratic production has been analyzed in $[14,15]$. Some unconditionally energy stable fully discrete schemes for a parabolic repulsive-productive chemotaxis model (with linear production term) were recently analyzed in [13]. On the other hand, when the interaction with a fluid is assumed, as far as we know, the literature related to the numerical analysis of chemotaxis-Navier-Stokes system is scarce, see [3,22]. In [22], some numerical evidence that solutions 
to the elliptic-parabolic 2D-Keller-Segel-Stokes system exist for initial mass larger than $8 \pi$, was considered. Even for the 2D-Keller-Segel-Stokes, the convergence of a numerical scheme given by the combination of the finite volume method and the nonconforming FE method has been studied in [3]. For the chemotaxis-NavierStokes system (1.1) (with other boundary conditions), we only know that some numerical simulations have been made in order to investigate the patterns formation and predict numerically the nonlinear dynamic of the chemotaxis-fluid system (see for instance, $[4,6,19,21,30]$ ).

This paper is focused on the proof of optimal convergence rates for a FE method approaching the solutions of (1.1) and (1.2). The main difficulties to deal with the numerical analysis of (1.1) and (1.2) come from the strong coupling nonlinear term $\nabla \cdot(\eta \nabla c)$ and the transport terms $\mathbf{u} \cdot \nabla \eta, \mathbf{u} \cdot \nabla c$ and $\mathbf{u} \cdot \nabla \mathbf{u}$. Therefore, in order to use the Finite Element method and deal with the chemotaxis term $\nabla \cdot(\eta \nabla c)$ we introduce a new variable given by the gradient of the chemical concentration, that is, $\boldsymbol{\sigma}=\nabla c$, which allows us to control the strong regularity requested by the system. On the other hand, the transport terms have the difficulty that the corresponding discrete forms do not preserve the alternance property as in the continuous case, and we need to introduce some skew-symmetric trilinear forms (see (3.9) and (3.10)). We concentrate our analysis by introducing a splitting FE method for approximating the weak solutions of (1.1) and (1.2) and analyze optimal error estimates. This scheme is well-posed and mass-conservative. We obtain uniform estimates and analyze the convergence towards regular solutions. We also present some numerical simulations in order to validate the theoretical results.

The plan of this paper is as follows. In Section 2, we first establish some basic notations and recall some existence and uniqueness results of (1.1) and (1.2) in the continuous case. We also define the weak formulation of (1.1) and (1.2), which will be used to construct the numerical approximation. In Section 3, we construct a mass-conservative numerical approximation for the weak solutions of the chemotaxis-fluid system (1.1) with initial and boundary data (1.2), by using FE approximations in space and finite differences in time, and give some preliminary results concerning the FE spaces. In Section 4, we analyze the well-posedness and the massconservation property (see (2.10)) for the numerical scheme; and following an inductive procedure, in Section 5, we obtain some uniform estimates for any solution of scheme (3.15), subsequently required in the convergence analysis. The analysis of Section 5 is focused on the two-dimensional case. In Section 6 , we make some comments about the extensions of these results to the three-dimensional case. Finally, in Section 7, we provide some numerical simulations in agreement with the theoretical results.

\section{The CONTINUOUS PROBLEM}

In this section, we establish a variational formulation of (1.1) and (1.2) which will be used to construct the numerical scheme. In order to deal with the chemotaxis term in the cell density equation, we introduce a splitting variational formulation for the chemoattractant concentration, which yields to consider an auxiliary variable representing the gradient of the chemical signal. In this section, after establishing the basic notations which will be used, we recall some existence results for two and three dimensional bounded domains, and define a variational formulation of the continuous problem.

\subsection{Notations}

We consider the standard Sobolev and Lebesgue spaces $W^{k, p}(\Omega)$ and $L^{p}(\Omega)$, with respective norms $\|\cdot\|_{W^{k, p}}$ and $\|\cdot\|_{L^{p}}$. In particular, we denote $W^{k, 2}(\Omega)=H^{k}(\Omega)$. Also, $W_{0}^{1, p}(\Omega)$ denotes the elements of $W^{1, p}(\Omega)$ with trace zero on the boundary of $\Omega$. The $L^{2}(\Omega)$-inner product will be represented by $(\cdot, \cdot)$. Corresponding Sobolev spaces of vector valued functions will be denoted by $\mathbf{W}^{k, p}(\Omega), \mathbf{L}^{p}(\Omega)$, and so on. Also, we will use the following function spaces

$$
\begin{aligned}
\mathbf{V} & :=\left\{\mathbf{v} \in \mathbf{H}_{0}^{1}(\Omega): \nabla \cdot \mathbf{v}=0 \quad \text { in } \quad \Omega\right\}, \\
\mathbf{H}_{\sigma}^{1}(\Omega) & :=\left\{\mathbf{v} \in \mathbf{H}^{1}(\Omega): \mathbf{v} \cdot \boldsymbol{\nu}=0 \quad \text { on } \quad \partial \Omega\right\},
\end{aligned}
$$




$$
\begin{aligned}
L_{0}^{2}(\Omega) & :=\left\{p \in L^{2}(\Omega): \int_{\Omega} p=0\right\}, \\
\widehat{H}^{1}(\Omega) & :=H^{1}(\Omega) \cap L_{0}^{2}(\Omega)=\left\{w \in H^{1}(\Omega): \int_{\Omega} w=0\right\},
\end{aligned}
$$

where $\boldsymbol{\nu}$ denotes the unit outward normal vector to the boundary. We will use the following equivalent norms in $H^{1}(\Omega)$ and $\mathbf{H}_{\sigma}^{1}(\Omega)$, respectively (see [25] and [1], Cor. 3.5):

$$
\begin{array}{ll}
\|u\|_{H^{1}}^{2}=\|\nabla u\|_{L^{2}}^{2}+\left(\int_{\Omega} u\right)^{2}, & \forall u \in H^{1}(\Omega), \\
\|\boldsymbol{\sigma}\|_{H^{1}}^{2}=\|\boldsymbol{\sigma}\|_{L^{2}}^{2}+\|\operatorname{rot} \boldsymbol{\sigma}\|_{L^{2}}^{2}+\|\nabla \cdot \boldsymbol{\sigma}\|_{L^{2}}^{2}, & \forall \boldsymbol{\sigma} \in \mathbf{H}_{\sigma}^{1}(\Omega) .
\end{array}
$$

We recall the well known Poincaré and embedding inequalities

$$
\begin{array}{ll}
\|\mathbf{v}\|_{H^{1}} \leq C_{P}\|\nabla \mathbf{v}\|_{L^{2}}, & \forall \mathbf{v} \in \mathbf{H}_{0}^{1}(\Omega), \\
\|u\|_{L^{\infty}} \leq C\|u\|_{H^{2}}, & \forall u \in H^{2}(\Omega),
\end{array}
$$

for some constants $C_{P}, C>0$, which depend on $\Omega$, but are independent of $\mathbf{v}$ and $u$, respectively. Also, we will use the classical interpolation inequalities

$$
\begin{aligned}
& \|u\|_{L^{4}} \leq C\|u\|_{L^{2}}^{1 / 2}\|u\|_{H^{1}}^{1 / 2} \quad \forall u \in H^{1}(\Omega) \quad \text { (in 2D domains), } \\
& \|u\|_{L^{3}} \leq\|u\|_{L^{2}}^{1 / 2}\|u\|_{L^{6}}^{1 / 2} \quad \forall u \in H^{1}(\Omega) \quad \text { (in 3D domains). }
\end{aligned}
$$

Finally, the letter $C$ denotes different positive constants (independent of discrete parameters) which may change from line to line (or even within the same line).

\subsection{Variational formulation}

In this subsection we give a variational formulation for (1.1) and (1.2). We start by recalling a result in [31] which provides the existence and uniqueness of global classical solution for (1.1) and (1.2) in bounded and convex domains of $\mathbb{R}^{2}$ with boundary $\partial \Omega$ smooth enough. Let us assume that the initial data satisfy the following regularity conditions:

$$
\begin{cases}\eta_{0} \in C^{0}(\bar{\Omega}), & \eta_{0}>0 \text { in } \bar{\Omega} \\ c_{0} \in W^{1, q}(\Omega) & \text { for some } q>2, \quad c_{0}>0 \quad \text { in } \bar{\Omega}, \\ \mathbf{u}_{0} \in D\left(A^{\alpha}\right) & \text { for some } \alpha \in\left(\frac{1}{2}, 1\right), \quad \nabla \phi \in C^{1}(\bar{\Omega}),\end{cases}
$$

where $A^{\alpha}$ denotes the fractional Stokes operator with domain $D\left(A^{\alpha}\right)$. Then, the following theorem holds.

Theorem 2.1. Let $\Omega \subset \mathbb{R}^{2}$ be a bounded domain with smooth boundary, and suppose that $\eta_{0}, c_{0}, \mathbf{u}_{0}, \phi$ satisfy (2.7). Then (1.1) and (1.2) possesses a classical solution which is global in time. This solution is unique, up to addition of constants to the pressure $\pi$, and for all $T \in(0, \infty)$ the solution has the following regularity properties

$$
\left\{\begin{array}{l}
\eta \in C\left([0, T) ; L^{2}(\Omega)\right) \cap L^{\infty}((0, T) ; C(\bar{\Omega})) \cap C^{2,1}(\bar{\Omega} \times(0, T)), \\
c \in C\left([0, T) ; L^{2}(\Omega)\right) \cap L^{\infty}\left((0, T) ; W^{1, q}(\Omega)\right) \cap C^{2,1}(\bar{\Omega} \times(0, T)), \\
\mathbf{u} \in C\left([0, T) ; L^{2}(\Omega)\right) \cap L^{\infty}\left((0, T) ; D\left(A^{\alpha}\right)\right) \cap C^{2,1}(\bar{\Omega} \times(0, T)), \\
\pi \in L^{1}\left((0, T) ; W^{1,2}\right) .
\end{array}\right.
$$

Proof. The proof is given in Theorem 1.1 of [31]. Indeed, the proof of [31] requires that $\Omega$ is a bounded convex domain. This condition comes from an integration term on the boundary which appears dealing with the differentiation of $\int_{\Omega}|\nabla \sqrt{c}|^{2}$. That boundary term takes the form $\int_{\partial \Omega} \frac{\partial}{\partial \nu}|\nabla c|^{2} \mathrm{~d} S$. Thus, if $\Omega$ is convex, the last boundary integral turns out to be non-positive and thus, this term can be neglected. However, the convexity condition can be removed by using Lemma 4.2 in [24], as used in [18,20]. 
For the three-dimensional setting of (1.1) and (1.2) and large initial data, Winkler in [33] proved the existence of weak solutions (for bounded convex domains). The notion of weak solution considered in [33] is the following one:

Definition 2.2 (Weak solution). A weak solution of (1.1) and (1.2) is a triple $[\eta, c, \mathbf{u}]$ of functions such that $\eta \in L^{1}\left([0, T], W^{1,1}(\Omega)\right), c \in L^{1}\left([0, T], W^{1,1}(\Omega)\right), \mathbf{u} \in L^{1}\left([0, T], W^{1,1}\right)$, verifying $\eta \geq 0$ and $c \geq 0$ a.e. in $\Omega \times(0, T)$, $\mathbf{u} \otimes \mathbf{u}, \eta \nabla c, \eta c$ and $\eta \mathbf{u} \in L^{1}\left(0, T ; L^{1}(\Omega)\right), \nabla \cdot \mathbf{u}=0$ a.e. in $\Omega \times(0, T)$, and such that

$$
\begin{array}{r}
-\int_{0}^{T} \int_{\Omega} \eta \varphi_{t}-\int_{\Omega} \eta_{0} \varphi(\cdot, 0)-\int_{0}^{T} \int_{\Omega} \eta \mathbf{u} \cdot \nabla \varphi+D_{n} \int_{0}^{T} \int_{\Omega} \nabla \eta \cdot \nabla \varphi=\chi \int_{0}^{T} \int_{\Omega} \eta \nabla c \cdot \nabla \varphi, \\
-\int_{0}^{T} \int_{\Omega} c \varphi_{t}-\int_{\Omega} c_{0} \varphi(\cdot, 0)-\int_{0}^{T} \int_{\Omega} c \mathbf{u} \cdot \nabla \varphi+D_{c} \int_{0}^{T} \int_{\Omega} \nabla c \cdot \nabla \varphi=-\gamma \int_{0}^{T} \int_{\Omega} \eta c \varphi, \\
-\rho \int_{0}^{T} \int_{\Omega} \mathbf{u} \cdot \psi_{t}-\rho \int_{\Omega} \mathbf{u}_{0} \cdot \boldsymbol{\psi}(\cdot, 0)-\rho \int_{0}^{T} \int_{\Omega} \mathbf{u} \otimes \mathbf{u} \cdot \nabla \boldsymbol{\psi}+D_{\mathbf{u}} \int_{0}^{T} \int_{\Omega} \nabla \mathbf{u} \cdot \nabla \boldsymbol{\psi}=\int_{0}^{T} \int_{\Omega} \eta \nabla \phi \cdot \boldsymbol{\psi},
\end{array}
$$

for all $\varphi \in C_{0}^{\infty}([0, T] \times \bar{\Omega})$ with $\varphi(T)=0$, and $\boldsymbol{\psi}$ in $C_{0}^{\infty}([0, T] \times \bar{\Omega})^{3}$ with $\boldsymbol{\psi}(T)=0$.

By assuming $\phi \in W^{2, \infty}(\Omega)$ and the initial data $\left[\eta_{0}, c_{0}, \mathbf{u}_{0}\right]$ satisfying

$$
\left\{\begin{array}{l}
\eta_{0} \in L \log L(\Omega) \text { is nonnegative with } \eta_{0} \neq 0 \\
c_{0} \in L^{\infty}(\Omega) \quad \text { is nonnegative with } \sqrt{c_{0}} \in W^{1,2}(\Omega), \\
\mathbf{u}_{0} \in L^{2}(\Omega), \quad \nabla \cdot \mathbf{u}_{0}=0,
\end{array}\right.
$$

in [33], Winkler proves the following theorem of global existence of weak solutions.

Theorem 2.3. Assume that the initial data $\left[\eta_{0}, c_{0}, \mathbf{u}_{0}\right]$ satisfies (2.9) and $\phi \in W^{2, \infty}(\Omega)$. Then there exist $\eta \in L^{\infty}\left(0, T ; L^{1}(\Omega)\right) \cap L^{\frac{5}{4}}\left([0, T], W^{1, \frac{5}{4}}(\Omega)\right), c \in L^{\infty}\left(0, T ; L^{\infty}(\Omega)\right) \cap L^{4}\left([0, T] ; W^{1,4}(\Omega)\right), \mathbf{u} \in L^{2}([0, T], \mathbf{V})$, such that $[\eta, c, \mathbf{u}]$ is a weak solution of (1.1) and (1.2) in the sense of Definition 2.2.

It is well known that system (1.1) and (1.2) conserves in time the total mass of bacteria (cf. [27]), that is,

$$
\int_{\Omega} \eta(\cdot, t) \mathrm{d} \boldsymbol{x}=\int_{\Omega} \eta_{0} \mathrm{~d} \boldsymbol{x}
$$

In order to design the scheme, taking into account the property (2.10), we first consider the auxiliary variable $n:=\eta-\frac{1}{|\Omega|} \int_{\Omega} \eta=\eta-\frac{1}{|\Omega|} \int_{\Omega} \eta_{0}:=\eta-\alpha_{0}$. Also, in order to control numerically the second order nonlinear term in the density equation, we will use the idea introduced by [36], in which the auxiliary variable $\sigma=\nabla c$ is considered, which will lead us to find one FE scheme with less restrictions relating the discrete parameters, in order to develop the convergence analysis (see Rem. 5.10). Thus, we obtain the following mixed variational form for the variables $n, c, \boldsymbol{\sigma}, \mathbf{u}$ and $\pi$ :

$$
\begin{cases}\left(n_{t}, \bar{n}\right)+D_{n}(\nabla n, \nabla \bar{n})+(\mathbf{u} \cdot \nabla n, \bar{n})=\chi\left(\left(n+\alpha_{0}\right) \boldsymbol{\sigma}, \nabla \bar{n}\right), & \forall \bar{n} \in \widehat{H}^{1}(\Omega), \\ (\boldsymbol{\sigma}, \overline{\boldsymbol{\sigma}})+(c, \nabla \cdot \overline{\boldsymbol{\sigma}})=0, & \forall \overline{\boldsymbol{\sigma}} \in \mathbf{H}_{\sigma}^{1}(\Omega), \\ \left(c_{t}, \bar{c}\right)-D_{c}(\nabla \cdot \boldsymbol{\sigma}, \bar{c})+(\mathbf{u} \cdot \boldsymbol{\sigma}, \bar{c})=-\gamma\left(\left(n+\alpha_{0}\right) c, \bar{c}\right), & \forall \bar{c} \in L^{2}(\Omega), \\ \rho\left(\mathbf{u}_{t}, \overline{\mathbf{u}}\right)+D_{\mathbf{u}}(\nabla \mathbf{u}, \nabla \overline{\mathbf{u}})+\rho((\mathbf{u} \cdot \nabla) \mathbf{u}, \overline{\mathbf{u}})-(\pi, \nabla \cdot \overline{\mathbf{u}})=\left(\left(n+\alpha_{0}\right) \nabla \phi, \overline{\mathbf{u}}\right), & \forall \overline{\mathbf{u}} \in \mathbf{H}_{0}^{1}(\Omega), \\ (\bar{\pi}, \nabla \cdot \mathbf{u})=0, & \forall \bar{\pi} \in L_{0}^{2}(\Omega) .\end{cases}
$$

Taking the time derivative in the equation $(2.11)_{2}$ we have

$$
\left(\boldsymbol{\sigma}_{t}, \overline{\boldsymbol{\sigma}}\right)+\left(c_{t}, \nabla \cdot \overline{\boldsymbol{\sigma}}\right)=0, \quad \forall \overline{\boldsymbol{\sigma}} \in \mathbf{H}_{\sigma}^{1}(\Omega) .
$$


Then, choosing $\bar{c}=\nabla \cdot \overline{\boldsymbol{\sigma}}$ in $(2.11)_{3}$, replacing it into (2.12), and adding the term $D_{c}(\operatorname{rot} \boldsymbol{\sigma}, \operatorname{rot} \overline{\boldsymbol{\sigma}})$ using that $\operatorname{rot} \boldsymbol{\sigma}=\operatorname{rot}(\nabla c)=0$, we get the following variational formulation:

$$
\left\{\begin{array}{l}
\left(n_{t}, \bar{n}\right)+D_{n}(\nabla n, \nabla \bar{n})+(\mathbf{u} \cdot \nabla n, \bar{n})=\chi\left(\left(n+\alpha_{0}\right) \boldsymbol{\sigma}, \nabla \bar{n}\right) \\
\left(\boldsymbol{\sigma}_{t}, \overline{\boldsymbol{\sigma}}\right)+D_{c}(\nabla \cdot \boldsymbol{\sigma}, \nabla \cdot \overline{\boldsymbol{\sigma}})+D_{c}(\operatorname{rot} \boldsymbol{\sigma}, \operatorname{rot} \overline{\boldsymbol{\sigma}})-(\mathbf{u} \cdot \boldsymbol{\sigma}, \nabla \cdot \overline{\boldsymbol{\sigma}})=\gamma\left(\left(n+\alpha_{0}\right) c, \nabla \cdot \overline{\boldsymbol{\sigma}}\right) \\
\left(c_{t}, \bar{c}\right)+D_{c}(\nabla c, \nabla \bar{c})+(\mathbf{u} \cdot \nabla c, \bar{c})=-\gamma\left(\left(n+\alpha_{0}\right) c, \bar{c}\right), \\
\rho\left(\mathbf{u}_{t}, \overline{\mathbf{u}}\right)+D_{\mathbf{u}}(\nabla \mathbf{u}, \nabla \overline{\mathbf{u}})+\rho((\mathbf{u} \cdot \nabla) \mathbf{u}, \overline{\mathbf{u}})-(\pi, \nabla \cdot \overline{\mathbf{u}})=\left(\left(n+\alpha_{0}\right) \nabla \phi, \overline{\mathbf{u}}\right) \\
(\bar{\pi}, \nabla \cdot \mathbf{u})=0
\end{array}\right.
$$

for all $[\bar{n}, \overline{\boldsymbol{\sigma}}, \bar{c}, \overline{\mathbf{u}}, \bar{\pi}] \in \widehat{H}^{1}(\Omega) \times \mathbf{H}_{\sigma}^{1}(\Omega) \times H^{1}(\Omega) \times \mathbf{H}_{0}^{1}(\Omega) \times L_{0}^{2}(\Omega)$. Notice that (2.13) defines a variational form of (1.1) and (1.2). Thus, if $[n, c, \mathbf{u}, \pi]$ is a classical solution of (1.1) and (1.2), defining $\boldsymbol{\sigma}=\nabla c$, previous procedure implies that $[n, c, \boldsymbol{\sigma}, \mathbf{u}, \pi]$ satisfies (2.13). Reciprocally, if $[n, c, \boldsymbol{\sigma}, \mathbf{u}, \pi]$ is a smooth solution of (2.13), integrating by parts equation $(2.13)_{3}$ we get

$$
c_{t}-D_{c} \Delta c+\mathbf{u} \cdot \nabla c=-\gamma\left(n+\alpha_{0}\right) c \text { a.e. in } \Omega .
$$

Thus, computing the gradient in (2.14), testing by $\overline{\boldsymbol{\sigma}} \in \mathbf{H}_{\sigma}^{1}(\Omega)$, and subtracting the resulting equation from $(2.13)_{2}$ we obtain that $\mathbf{w}=\nabla c-\boldsymbol{\sigma}$ satisfies:

$$
\left(\mathbf{w}_{t}, \overline{\boldsymbol{\sigma}}\right)+D_{c}(\nabla \cdot \mathbf{w}, \nabla \cdot \overline{\boldsymbol{\sigma}})+D_{c}(\operatorname{rot} \mathbf{w}, \operatorname{rot} \overline{\boldsymbol{\sigma}})-(\mathbf{u} \cdot \mathbf{w}, \nabla \cdot \overline{\boldsymbol{\sigma}})=0 \quad \forall \overline{\boldsymbol{\sigma}} \in \mathbf{H}_{\sigma}^{1}(\Omega) .
$$

Then, taking $\mathbf{w} \in \mathbf{H}_{\sigma}^{1}(\Omega)$ as test function, we dedude that:

$$
\begin{aligned}
\frac{1}{2} \frac{\mathrm{d}}{\mathrm{d} t}\|\mathbf{w}\|_{L^{2}}^{2}+D_{c}\left(\|\nabla \cdot \mathbf{w}\|_{L^{2}}^{2}+\|\operatorname{rot} \mathbf{w}\|_{L^{2}}^{2}\right) & \leq(\mathbf{u} \cdot \mathbf{w}, \nabla \cdot \mathbf{w}) \leq\|\mathbf{u}\|_{L^{6}}\|\mathbf{w}\|_{L^{3}}\|\nabla \cdot \mathbf{w}\|_{L^{2}} \\
& \leq \varepsilon\|\nabla \cdot \mathbf{w}\|_{L^{2}}^{2}+C_{\varepsilon}\|\mathbf{u}\|_{L^{6}}^{2}\|\mathbf{w}\|_{L^{3}}^{2} \\
& \leq \varepsilon\|\nabla \cdot \mathbf{w}\|_{L^{2}}^{2}+C_{\varepsilon}\|\mathbf{u}\|_{L^{6}}^{2}\left(\|\mathbf{w}\|_{L^{2}}^{2}+\|\mathbf{w}\|_{L^{2}}\|\nabla \mathbf{w}\|_{L^{2}}\right) \\
& \leq \varepsilon\left(\|\nabla \cdot \mathbf{w}\|_{L^{2}}^{2}+\|\operatorname{rot} \mathbf{w}\|_{L^{2}}^{2}\right)+C_{\varepsilon}\left(\|\mathbf{u}\|_{L^{6}}^{4}+\|\mathbf{u}\|_{L^{6}}^{2}\right)\|\mathbf{w}\|_{L^{2}}^{2}
\end{aligned}
$$

for $\varepsilon>0$ small enough. Taking into account that $\mathbf{w}(0)=0$, we deduce that $\mathbf{w}=0$ and therefore $\boldsymbol{\sigma}=\nabla c$. Finally, replacing $\boldsymbol{\sigma}=\nabla c$ in $(2.13)_{1}$ and integrating by parts once again, we conclude that $[n, c, \mathbf{u}, \pi]$ is a smooth solution of (1.1) and (1.2). For more details, we refer [14] where a similar argument (without interaction with fluid) is presented.

\section{NumERICAL SCHEME}

In this section, we construct a mass-conservative numerical approximation for the weak solutions of the chemotaxis-fluid system (1.1) with initial and boundary data (1.2). The idea is to use Finite Element approximations in space and finite differences in time (considered for simplicity on a uniform partition of $[0, T]$ with time step $\left.\Delta t=T / N:\left(t_{m}=m \Delta t\right)_{m=0}^{m=N}\right)$, combined with splitting ideas to decouple the computation of the fluid part from the chemotaxis one. Moreover, in order to deal with the velocity trilinear form and the nonlinear convective terms, we will use the skew-symmetric forms $A$ and $B$ given in (3.9) and (3.10). Concerning the space discretization, we consider conforming FE spaces: $\mathcal{X}_{n} \times \mathcal{X}_{c} \times \mathcal{X}_{\boldsymbol{\sigma}} \times \mathcal{X}_{\mathbf{u}} \times \mathcal{X}_{\pi} \subset \widehat{H}^{1}(\Omega) \times H^{1}(\Omega) \times \mathbf{H}_{\sigma}^{1}(\Omega) \times \mathbf{H}_{0}^{1}(\Omega) \times L_{0}^{2}(\Omega)$ corresponding to a family of shape-regular and quasi-uniform triangulations of $\bar{\Omega},\left\{\mathcal{T}_{h}\right\}_{h>0}$, made up of simplexes $K$ (triangles in $2 \mathrm{D}$ and tetrahedra en $3 \mathrm{D}$ ), so that $\bar{\Omega}=\cup_{K \in \mathcal{T}_{h}} K$, where $h=\max _{K \in \mathcal{T}_{h}} h_{K}$, with $h_{K}$ being the diameter of $K$. 


\subsection{Hyphoteses and preliminary results}

We assume that $\mathcal{X}_{\mathbf{u}}$ and $\mathcal{X}_{\pi}$ satisfy the following discrete inf-sup condition: There exists a constant $\beta>0$, independent of $h$, such that

$$
\sup _{\mathbf{v} \in \mathcal{X}_{\mathbf{u}} \backslash\{0\}} \frac{-(w, \nabla \cdot \mathbf{v})}{\|\mathbf{v}\|_{\mathcal{X}_{\mathbf{u}}}} \geq \beta\|w\|_{\mathcal{X}_{\pi}}, \quad \forall w \in \mathcal{X}_{\pi} .
$$

Remark 3.1 (Some possibilities for the choice of the discrete spaces). For the spaces $\left[\mathcal{X}_{\mathbf{u}}, \mathcal{X}_{\pi}\right]$, we can choose the Taylor-Hood approximation $\mathbb{P}_{r} \times \mathbb{P}_{r-1}($ for $r \geq 2)([11,28])$; or the approximation $\mathbb{P}_{1}-$ bubble $\times \mathbb{P}_{1}([11])$ (for $r=1$ ). On the other hand, the spaces $\left[\mathcal{X}_{n}, \mathcal{X}_{c}, \mathcal{X}_{\boldsymbol{\sigma}}\right]$ are approximated by $\mathbb{P}_{r_{1}} \times \mathbb{P}_{r_{2}} \times \mathbb{P}_{r_{3}}-$ continuous FE, with $r_{i} \geq 1(i=1,2,3)$.

Then, we can define the well-known Stokes operator $\left(\mathbb{P}_{\mathbf{u}}, \mathbb{P}_{\pi}\right): \mathbf{H}_{0}^{1}(\Omega) \times L_{0}^{2}(\Omega) \rightarrow \mathcal{X}_{\mathbf{u}} \times \mathcal{X}_{\pi}$ such that $\left[\mathbb{P}_{\mathbf{u}} \mathbf{u}, \mathbb{P}_{\pi} \pi\right] \in \mathcal{X}_{\mathbf{u}} \times \mathcal{X}_{\pi}$ satisfies

$$
\begin{cases}D_{\mathbf{u}}\left(\nabla\left(\mathbb{P}_{\mathbf{u}} \mathbf{u}-\mathbf{u}\right), \nabla \overline{\mathbf{u}}\right)-\left(\mathbb{P}_{\pi} \pi-\pi, \nabla \cdot \overline{\mathbf{u}}\right)=0, & \forall \overline{\mathbf{u}} \in \mathcal{X}_{\mathbf{u}} \\ \left(\nabla \cdot\left(\mathbb{P}_{\mathbf{u}} \mathbf{u}-\mathbf{u}\right), \bar{\pi}\right)=0, & \forall \bar{\pi} \in \mathcal{X}_{\pi}\end{cases}
$$

and the following approximation and stability properties hold ([12]):

$$
\begin{aligned}
\left\|\left[\mathbf{u}-\mathbb{P}_{\mathbf{u}} \mathbf{u}, \pi-\mathbb{P}_{\pi} \pi\right]\right\|_{H^{1} \times L^{2}}+\frac{1}{h}\left\|\mathbf{u}-\mathbb{P}_{\mathbf{u}} \mathbf{u}\right\|_{L^{2}} & \leq K h^{r}\|[\mathbf{u}, \pi]\|_{H^{r+1} \times H^{r}}, \\
\left\|\left[\mathbb{P}_{\mathbf{u}} \mathbf{u}, \mathbb{P}_{\pi} \pi\right]\right\|_{W^{1,6} \times L^{6}} & \leq C\|[\mathbf{u}, \pi]\|_{H^{2} \times H^{1}} .
\end{aligned}
$$

Moreover, we consider the following interpolation operators:

$$
\mathbb{P}_{n}: \widehat{H}^{1}(\Omega) \rightarrow \mathcal{X}_{n}, \quad \mathbb{P}_{c}: H^{1}(\Omega) \rightarrow \mathcal{X}_{c}, \quad \mathbb{P}_{\boldsymbol{\sigma}}: \mathbf{H}_{\sigma}^{1}(\Omega) \rightarrow \mathcal{X}_{\boldsymbol{\sigma}}
$$

such that for all $n \in \widehat{H}^{1}(\Omega), c \in H^{1}(\Omega)$ and $\boldsymbol{\sigma} \in \mathbf{H}_{\sigma}^{1}(\Omega), \mathbb{P}_{n} n \in \mathcal{X}_{n}, \mathbb{P}_{c} c \in \mathcal{X}_{c}$ and $\mathbb{P}_{\boldsymbol{\sigma}} \boldsymbol{\sigma} \in \mathcal{X}_{\boldsymbol{\sigma}}$ satisfy

$$
\begin{cases}\left(\nabla\left(\mathbb{P}_{n} n-n\right), \nabla \bar{n}\right)=0, & \forall \bar{n} \in \mathcal{X}_{n}, \\ \left(\nabla\left(\mathbb{P}_{c} c-c\right), \nabla \bar{c}\right)+\left(\mathbb{P}_{c} c-c, \bar{c}\right)=0, & \forall \bar{c} \in \mathcal{X}_{c}, \\ \left(\nabla \cdot\left(\mathbb{P}_{\boldsymbol{\sigma}} \boldsymbol{\sigma}-\boldsymbol{\sigma}\right), \nabla \cdot \overline{\boldsymbol{\sigma}}\right)+\left(\operatorname{rot}\left(\mathbb{P}_{\boldsymbol{\sigma}} \boldsymbol{\sigma}-\boldsymbol{\sigma}\right), \operatorname{rot} \overline{\boldsymbol{\sigma}}\right)+\left(\mathbb{P}_{\boldsymbol{\sigma}} \boldsymbol{\sigma}-\boldsymbol{\sigma}, \overline{\boldsymbol{\sigma}}\right)=0, & \forall \overline{\boldsymbol{\sigma}} \in \mathcal{X}_{\boldsymbol{\sigma}},\end{cases}
$$

respectively. Observe that from Lax-Milgram theorem, we have that the interpolation operators $\mathbb{P}_{n}, \mathbb{P}_{c}$ and $\mathbb{P}_{\boldsymbol{\sigma}}$ are well defined. Moreover, it is well known that the following interpolation errors hold:

$$
\begin{cases}\left\|n-\mathbb{P}_{n} n\right\|_{L^{2}}+h\left\|n-\mathbb{P}_{n} n\right\|_{H^{1}} \leq K h^{r_{1}+1}\|n\|_{H^{r_{1}+1}}, & \forall n \in H^{r_{1}+1}(\Omega), \\ \left\|c-\mathbb{P}_{c} c\right\|_{L^{2}}+h\left\|c-\mathbb{P}_{c} c\right\|_{H^{1}} \leq K h^{r_{2}+1}\|c\|_{H^{r_{2}+1}}, & \forall c \in H^{r_{2}+1}(\Omega), \\ \left\|\boldsymbol{\sigma}-\mathbb{P}_{\boldsymbol{\sigma}} \boldsymbol{\sigma}\right\|_{L^{2}}+h\left\|\boldsymbol{\sigma}-\mathbb{P}_{\boldsymbol{\sigma}} \boldsymbol{\sigma}\right\|_{H^{1}} \leq C h^{r_{3}+1}\|\boldsymbol{\sigma}\|_{H^{r_{3}+1}}, & \forall \boldsymbol{\sigma} \in \mathbf{H}^{r_{3}+1}(\Omega) .\end{cases}
$$

Also, the stability properties

$$
\begin{aligned}
\left\|\left[\mathbb{P}_{n} n, \mathbb{P}_{c} c, \mathbb{P}_{\boldsymbol{\sigma}} \boldsymbol{\sigma}\right]\right\|_{H^{1}} & \leq\|[n, c, \boldsymbol{\sigma}]\|_{H^{1}}, \\
\left\|\left[\mathbb{P}_{n} n, \mathbb{P}_{c} c, \mathbb{P}_{\boldsymbol{\sigma}} \boldsymbol{\sigma}\right]\right\|_{W^{1,6}} & \leq C\|[n, c, \boldsymbol{\sigma}]\|_{H^{2}}
\end{aligned}
$$

hold. Inequality (3.7) can be deduced from (3.5), and (3.8) can be obtained from (3.6) using the inverse inequality

$$
\left\|\left[n_{h}, c_{h}, \boldsymbol{\sigma}_{h}\right]\right\|_{W^{1,6}} \leq C h^{-p}\left\|\left[n_{h}, c_{h}, \boldsymbol{\sigma}_{h}\right]\right\|_{H^{1}} \quad \text { for all }\left[n_{h}, c_{h}, \boldsymbol{\sigma}_{h}\right] \in \mathbb{P}_{n} \times \mathbb{P}_{c} \times \mathbb{P}_{\boldsymbol{\sigma}}
$$

with $p=2 / 3$ (in the $2 \mathrm{D}$ case) and $p=1$ (in the $3 \mathrm{D}$ case), and comparing $\mathbb{P}_{n, c, \boldsymbol{\sigma}}$ with an average interpolation of Clement or Scott-Zhang type (which are stable in $W^{1,6}$-norm). Finally, we consider the following skew-symmetric trilinear forms which will be used in the formulation of the numerical scheme:

$$
B\left(\mathbf{v}_{1}, \mathbf{v}_{2}, \mathbf{v}_{3}\right)=\frac{1}{2}\left[\left(\left(\mathbf{v}_{1} \cdot \nabla\right) \mathbf{v}_{2}, \mathbf{v}_{3}\right)-\left(\left(\mathbf{v}_{1} \cdot \nabla\right) \mathbf{v}_{3}, \mathbf{v}_{2}\right)\right], \quad \forall \mathbf{v}_{1}, \mathbf{v}_{2}, \mathbf{v}_{3} \in \mathbf{H}^{1}(\Omega),
$$




$$
A\left(\mathbf{v}, w_{1}, w_{2}\right)=\frac{1}{2}\left[\left((\mathbf{v} \cdot \nabla) w_{1}, w_{2}\right)-\left((\mathbf{v} \cdot \nabla) w_{2}, w_{1}\right)\right], \quad \forall \mathbf{v} \in \mathbf{H}^{1}(\Omega), w_{1}, w_{2} \in H^{1}(\Omega) .
$$

It is easy to verify that

$$
\begin{array}{rlrlrl}
B\left(\mathbf{v}_{1}, \mathbf{v}_{2}, \mathbf{v}_{3}\right) & =\left(\left(\mathbf{v}_{1} \cdot \nabla\right) \mathbf{v}_{2}, \mathbf{v}_{3}\right), & & \forall \mathbf{v}_{1} \in \mathbf{V}, & \mathbf{v}_{2}, \mathbf{v}_{3} \in \mathbf{H}^{1}(\Omega), \\
A\left(\mathbf{v}_{1}, w_{1}, w_{2}\right) & =\left(\left(\mathbf{v}_{1} \cdot \nabla\right) w_{1}, w_{2}\right), & & \forall \mathbf{v}_{1} \in \mathbf{V}, & & w_{1}, w_{2} \in H^{1}(\Omega), \\
B\left(\mathbf{v}_{1}, \mathbf{v}_{2}, \mathbf{v}_{2}\right) & =0, & & \forall \mathbf{v}_{1}, \mathbf{v}_{2} \in \mathbf{H}^{1}(\Omega), & & \\
A(\mathbf{v}, w, w) & =0, & & \forall w \in H^{1}(\Omega), & \mathbf{v} \in \mathbf{H}^{1}(\Omega) .
\end{array}
$$

\subsection{Definition of the scheme}

Taking into account (2.13), we consider the following first order in time, linear and semi-coupled scheme:

Initialization. Let $\left[n_{h}^{0}, c_{h}^{0}, \boldsymbol{\sigma}_{h}^{0}, \mathbf{u}_{h}^{0}\right]=\left[\mathbb{P}_{n} n_{0}, \mathbb{P}_{c} c_{0}, \mathbb{P}_{\boldsymbol{\sigma}} \boldsymbol{\sigma}_{0}, \mathbb{P}_{\mathbf{u}} \mathbf{u}_{0}\right] \in \mathcal{X}_{n} \times \mathcal{X}_{c} \times \mathcal{X}_{\boldsymbol{\sigma}} \times \mathcal{X}_{\mathbf{u}}$

Time step $m$. Given the vector $\left[n_{h}^{m-1}, c_{h}^{m-1}, \boldsymbol{\sigma}_{h}^{m-1}, \mathbf{u}_{h}^{m-1}\right] \in \mathcal{X}_{n} \times \mathcal{X}_{c} \times \mathcal{X}_{\boldsymbol{\sigma}} \times \mathcal{X}_{\mathbf{u}}$, compute $\left[n_{h}^{m}, c_{h}^{m}, \boldsymbol{\sigma}_{h}^{m}, \mathbf{u}_{h}^{m}, \pi_{h}^{m}\right] \in$ $\mathcal{X}_{n} \times \mathcal{X}_{c} \times \mathcal{X}_{\boldsymbol{\sigma}} \times \mathcal{X}_{\mathbf{u}} \times \mathcal{X}_{\pi}$ such that for each $[\bar{n}, \bar{c}, \overline{\boldsymbol{\sigma}}, \overline{\mathbf{u}}, \bar{\pi}] \in \mathcal{X}_{n} \times \mathcal{X}_{c} \times \mathcal{X}_{\boldsymbol{\sigma}} \times \mathcal{X}_{\mathbf{u}} \times \mathcal{X}_{\pi}$ it holds:

(a) $\left(\delta_{t} n_{h}^{m}, \bar{n}\right)+D_{n}\left(\nabla n_{h}^{m}, \nabla \bar{n}\right)+A\left(\mathbf{u}_{h}^{m-1}, n_{h}^{m}, \bar{n}\right)-\chi\left(\left(n_{h}^{m-1}+\alpha_{0}\right) \boldsymbol{\sigma}_{h}^{m-1}, \nabla \bar{n}\right)=0$,

(b) $\left(\delta_{t} \boldsymbol{\sigma}_{h}^{m}, \overline{\boldsymbol{\sigma}}\right)+D_{c}\left(\nabla \cdot \boldsymbol{\sigma}_{h}^{m}, \nabla \cdot \overline{\boldsymbol{\sigma}}\right)+D_{c}\left(\operatorname{rot} \boldsymbol{\sigma}_{h}^{m}, \operatorname{rot} \overline{\boldsymbol{\sigma}}\right)=\left(\mathbf{u}_{h}^{m-1} \cdot \boldsymbol{\sigma}_{h}^{m-1}+\gamma\left(n_{h}^{m-1}+\alpha_{0}\right) c_{h}^{m-1}, \nabla \cdot \overline{\boldsymbol{\sigma}}\right)$,

(c) $\left(\delta_{t} c_{h}^{m}, \bar{c}\right)+D_{c}\left(\nabla c_{h}^{m}, \nabla \bar{c}\right)+A\left(\mathbf{u}_{h}^{m-1}, c_{h}^{m}, \bar{c}\right)=-\gamma\left(\left(n_{h}^{m-1}+\alpha_{0}\right) c_{h}^{m-1}, \bar{c}\right)$,

(d) $\left(\delta_{t} \mathbf{u}_{h}^{m}, \overline{\mathbf{u}}\right)+B\left(\mathbf{u}_{h}^{m-1}, \mathbf{u}_{h}^{m}, \overline{\mathbf{u}}\right)+\frac{D_{\mathbf{u}}}{\rho}\left(\nabla \mathbf{u}_{h}^{m}, \nabla \overline{\mathbf{u}}\right)-\frac{1}{\rho}\left(\pi_{h}^{m}, \nabla \cdot \overline{\mathbf{u}}\right)=\frac{1}{\rho}\left(\left(n_{h}^{m-1}+\alpha_{0}\right) \nabla \phi, \overline{\mathbf{u}}\right)$,

(e) $\left(\nabla \cdot \mathbf{u}_{h}^{m}, \bar{\pi}\right)=0$,

where, in general, we denote $\delta_{t} a_{h}^{m}=\frac{a_{h}^{m}-a_{h}^{m-1}}{\Delta t}$.

Remark 3.2. The skew-symmetric forms $A$ and $B$ verifying the properties (3.11)-(3.14) will be important in order to get uniform estimates for the discrete solutions and to develop convergence analysis.

\section{WELL-POSEDNESS AND MASS-CONSERVATION}

In this section, we analyze the well-posedness of the scheme (3.15) and the mass-conservation property. To this aim, we define $\eta_{h}^{m}:=n_{h}^{m}+\alpha_{0}$. Then $\eta_{h}^{m}$ verifies

$$
\left(\delta_{t} \eta_{h}^{m}, \bar{n}\right)+D_{n}\left(\nabla \eta_{h}^{m}, \nabla \bar{n}\right)+A\left(\mathbf{u}_{h}^{m-1}, \eta_{h}^{m}-\alpha_{0}, \bar{n}\right)-\chi\left(\eta_{h}^{m-1} \boldsymbol{\sigma}_{h}^{m-1}, \nabla \bar{n}\right)=0 .
$$

Lemma 4.1 (Mass conservation). The discrete cell density $\eta_{h}^{m}$ satisfies the mass-conservation property (2.10).

Proof. From the construction of scheme (3.15), for each $m \geq 0$ it holds that $\int_{\Omega} n_{h}^{m}=0$ (since $n_{h}^{m} \in \mathcal{X}_{n} \subset \widehat{H}^{1}(\Omega)$ ); therefore we deduce that $\eta_{h}^{m}$ satisfies

$$
\int_{\Omega} \eta_{h}^{m}=\int_{\Omega} \eta_{h}^{m-1}=\cdots=\int_{\Omega} \eta_{h}^{0}=\alpha_{0}
$$

Now, in the next result, we prove the well-posedness of the scheme (3.15).

Theorem 4.2 (Unconditional well-posedness). The numerical scheme (3.15) is well-posed, that is, there exists a unique $\left[n_{h}^{m}, c_{h}^{m}, \boldsymbol{\sigma}_{h}^{m}, \mathbf{u}_{h}^{m}, \pi_{h}^{m}\right] \in \mathcal{X}_{n} \times \mathcal{X}_{c} \times \mathcal{X}_{\boldsymbol{\sigma}} \times \mathcal{X}_{\mathbf{u}} \times \mathcal{X}_{\pi}$ solution of the scheme (3.15). 
Proof. Taking into account that the scheme (3.15) is an algebraic linear system, it suffices to prove the uniqueness. For that, suppose that there exist $\left[n_{h, 1}^{m}, c_{h, 1}^{m}, \boldsymbol{\sigma}_{h, 1}^{m}, \mathbf{u}_{h, 1}^{m}, \pi_{h, 1}^{m}\right],\left[n_{h, 2}^{m}, c_{h, 2}^{m}, \boldsymbol{\sigma}_{h, 2}^{m}, \mathbf{u}_{h, 2}^{m}, \pi_{h, 2}^{m}\right] \in \mathcal{X}_{n} \times \mathcal{X}_{c} \times \mathcal{X}_{\boldsymbol{\sigma}} \times$ $\mathcal{X}_{\mathbf{u}} \times \mathcal{X}_{\pi}$ two possible solutions of the scheme (3.15). Then defining $n_{h}^{m}=n_{h, 1}^{m}-n_{h, 2}^{m}, c_{h}^{m}=c_{h, 1}^{m}-c_{h, 2}^{m}, \boldsymbol{\sigma}_{h}^{m}=$ $\boldsymbol{\sigma}_{h, 1}^{m}-\boldsymbol{\sigma}_{h, 2}^{m} \mathbf{u}_{h}^{m}=\mathbf{u}_{h, 1}^{m}-\mathbf{u}_{h, 2}^{m}$ and $\pi_{h}^{m}=\pi_{h, 1}^{m}-\pi_{h, 2}^{m}$, we have that $\left[n_{h}^{m}, c_{h}^{m}, \boldsymbol{\sigma}_{h}^{m}, \mathbf{u}_{h}^{m}, \pi_{h}^{m}\right] \in \mathcal{X}_{n} \times \mathcal{X}_{c} \times \mathcal{X}_{\boldsymbol{\sigma}} \times \mathcal{X}_{\mathbf{u}} \times \mathcal{X}_{\pi}$ satisfies

$$
\left\{\begin{array}{l}
\left(n_{h}^{m}, \bar{n}\right)+\Delta t D_{n}\left(\nabla n_{h}^{m}, \nabla \bar{n}\right)+\Delta t A\left(\mathbf{u}_{h}^{m-1}, n_{h}^{m}, \bar{n}\right)=0, \\
\left(\boldsymbol{\sigma}_{h}^{m}, \overline{\boldsymbol{\sigma}}\right)+\Delta t D_{c}\left(\nabla \cdot \boldsymbol{\sigma}_{h}^{m}, \nabla \cdot \overline{\boldsymbol{\sigma}}\right)+\Delta t D_{c}\left(\operatorname{rot} \boldsymbol{\sigma}_{h}^{m}, \operatorname{rot} \overline{\boldsymbol{\sigma}}\right)=0, \\
\left(c_{h}^{m}, \bar{c}\right)+\Delta t D_{c}\left(\nabla c_{h}^{m}, \nabla \bar{c}\right)+\Delta t A\left(\mathbf{u}_{h}^{m-1}, c_{h}^{m}, \bar{c}\right)=0, \\
\rho\left(\mathbf{u}_{h}^{m}, \overline{\mathbf{u}}\right)+\Delta t \rho B\left(\mathbf{u}_{h}^{m-1}, \mathbf{u}_{h}^{m}, \overline{\mathbf{u}}\right)+\Delta t D_{\mathbf{u}}\left(\nabla \mathbf{u}_{h}^{m}, \nabla \overline{\mathbf{u}}\right)-\Delta t\left(\pi_{h}^{m}, \nabla \cdot \overline{\mathbf{u}}\right)=0, \\
\left(\bar{\pi}, \nabla \cdot \mathbf{u}_{h}^{m}\right)=0
\end{array}\right.
$$

for all $[\bar{n}, \bar{c}, \overline{\boldsymbol{\sigma}}, \overline{\mathbf{u}}, \bar{\pi}] \in \mathcal{X}_{n} \times \mathcal{X}_{c} \times \mathcal{X}_{\boldsymbol{\sigma}} \times \mathcal{X}_{\mathbf{u}} \times \mathcal{X}_{\pi}$. Taking $[\bar{n}, \bar{c}, \overline{\boldsymbol{\sigma}}, \overline{\mathbf{u}}, \bar{\pi}]=\left[n_{h}^{m}, c_{h}^{m}, \boldsymbol{\sigma}_{h}^{m}, \mathbf{u}_{h}^{m}, \Delta t \pi_{h}^{m}\right]$ in (4.1), using the properties (3.13) and (3.14), and adding, we obtain

$$
\left\{\begin{array}{l}
\left\|n_{h}^{m}\right\|_{L^{2}}^{2}+\Delta t D_{n}\left\|\nabla n_{h}^{m}\right\|_{L^{2}}^{2}=0, \\
\left\|\boldsymbol{\sigma}_{h}^{m}\right\|_{L^{2}}^{2}+\Delta t D_{c}\left\|\nabla \cdot \boldsymbol{\sigma}_{h}^{m}\right\|_{L^{2}}^{2}+\Delta t D_{c}\left\|\operatorname{rot} \boldsymbol{\sigma}_{h}^{m}\right\|_{L^{2}}^{2}=0 \\
\left\|c_{h}^{m}\right\|_{L^{2}}^{2}+\Delta t D_{c}\left\|\nabla c_{h}^{m}\right\|_{L^{2}}^{2}=0 \\
\rho\left\|\mathbf{u}_{h}^{m}\right\|_{L^{2}}^{2}+\Delta t D_{\mathbf{u}}\left\|\nabla \mathbf{u}_{h}^{m}\right\|_{L^{2}}^{2}=0,
\end{array}\right.
$$

which implies that $\left[n_{h}^{m}, c_{h}^{m}, \boldsymbol{\sigma}_{h}^{m}, \mathbf{u}_{h}^{m}\right]=[0,0, \mathbf{0}, \mathbf{0}]$. Finally, using that $\mathbf{u}_{h}^{m}=\mathbf{0}$ in $(4.1)_{4}$, and using the discrete inf-sup condition (3.1) we deduce that $\pi_{h}^{m}=0$.

\section{UNIFORM ESTIMATES AND CONVERGENCE}

In this section, we will obtain some uniform estimates for any solution of scheme (3.15) that will be used in the convergence analysis. Here, we focus on the two-dimensional case. Later, in Section 6, we study the threedimensional case. To this aim, we make the following inductive hypothesis: there exists a positive constant $K>0$, independent of $m$, such that

$$
\left\|\boldsymbol{\sigma}_{h}^{m-1}\right\|_{H^{1}} \leq K, \quad \forall m \geq 1 .
$$

After the convergence analysis we verify the vality of (5.1). It is worthwhile to remark that the use of induction hypothesis to deal with the convergence analysis of approximating schemes in nonlinear partial differential equations has been considered by several authors (see for instance, [5, 7,34,36]). In [7], Douglas and Roberts analyzed a numerical method for a model describing compressible miscible displacement in porous media, and considered an inductive hypothesis on the difference of pressures $\pi=p-p_{h}$ coming from the Darcy model, in norm $W^{1, \infty}$. In the same spirit, by using inductive hypotheses, a superconvergence estimate of a combined mixed FE and discontinuous Galerkin method for a compressible miscible displacement problem was obtained in [34] (see also [5]). In the context of the Keller-Segel system without fluid influence, in [36], an inductive hypothesis on $\left\|\boldsymbol{\sigma}_{h}^{m}\right\|_{W^{1, \infty}}$ is considered. Observe that the norm in $(5.1)$ is in $H^{1}(\Omega)$ instead of $W^{1, \infty}(\Omega)$.

Additionally, we will use the following discrete Gronwall lemma:

Lemma 5.1 ([16], p. 369). Assume that $\Delta t>0$ and $B, b^{k}, d^{k}, g^{k}, h^{k} \geq 0$ satisfy:

$$
d^{m+1}+\Delta t \sum_{k=0}^{m} b^{k+1} \leq \Delta t \sum_{k=0}^{m} g^{k} d^{k}+\Delta t \sum_{k=0}^{m} h^{k}+B, \quad \forall m \geq 0 .
$$

Then, it holds

$$
d^{m+1}+\Delta t \sum_{k=0}^{m} b^{k+1} \leq \exp \left(\Delta t \sum_{k=0}^{m} g^{k}\right)\left(\Delta t \sum_{k=0}^{m} h^{k}+B\right), \quad \forall m \geq 0
$$




\subsection{Uniform estimates in finite time}

We can prove the following uniform estimates in finite time for the discrete cell and chemical variables, $n_{h}^{m}$ and $c_{h}^{m}$, in weak norms.

Lemma 5.2 (Uniform estimates for $\left.n_{h}^{m}\right)$. Assume the inductive hypothesis (5.1). Then $n_{h}^{m}$ is bounded in $l^{\infty}\left(L^{2}\right) \cap l^{2}\left(H^{1}\right)$.

Proof. Testing (3.15) $)_{a}$ by $\bar{n}=2 \Delta t n_{h}^{m}$, using the equality $(a-b, 2 a)=|a|^{2}-|b|^{2}+|a-b|^{2}$, taking into account the property (3.14) and using the fact that $\int_{\Omega} n_{h}^{m}=0$ (since $n_{h}^{m} \in \mathcal{X}_{n}$ ), we have

$$
\left\|n_{h}^{m}\right\|_{L^{2}}^{2}-\left\|n_{h}^{m-1}\right\|_{L^{2}}^{2}+\left\|n_{h}^{m}-n_{h}^{m-1}\right\|_{L^{2}}^{2}+2 D_{n} \Delta t\left\|n_{h}^{m}\right\|_{H^{1}}^{2}=2 \Delta t \chi\left(\left(n_{h}^{m-1}+\alpha_{0}\right) \boldsymbol{\sigma}_{h}^{m-1}, \nabla n_{h}^{m}\right) .
$$

Using the Hölder and Young inequalities and the 2D interpolation inequality (2.5), we get

$$
\begin{aligned}
& 2 \Delta t \chi \\
& \left.\left.\quad \leq C n_{h}^{m-1}+\alpha_{0}\right) \boldsymbol{\sigma}_{h}^{m-1}, \nabla n_{h}^{m}\right) \leq C \Delta t \chi\left(\left\|n_{h}^{m-1}\right\|_{L^{4}}+\alpha_{0}\right)\left\|\boldsymbol{\sigma}_{h}^{m-1}\right\|_{L^{4}}\left\|\nabla n_{h}^{m}\right\|_{L^{2}} \\
& \quad \leq C t \chi\left(\left\|n_{h}^{m-1}\right\|_{L^{2}}^{1 / 2}\left\|n_{h}^{m-1}\right\|_{H^{1}}^{1 / 2}+\alpha_{0}\right)\left\|\boldsymbol{\sigma}_{h}^{m-1}\right\|_{L^{4}}\left\|\nabla n_{h}^{m}\right\|_{L^{2}} \\
& \quad \leq D_{n} \Delta t\left\|n_{h}^{m}\right\|_{H^{1}}^{2}+\frac{D_{n} \Delta t}{2}\left\|n_{h}^{m-1}\right\|_{H^{1}}^{2}+\frac{C \Delta t \chi^{2}}{D_{n}}\left\|\boldsymbol{\sigma}_{h}^{m-1}\right\|_{L^{4}}^{2}\left(\frac{\chi^{2}}{D_{n}^{2}}\left\|n_{h}^{m-1}\right\|_{L^{2}}^{2}\left\|\boldsymbol{\sigma}_{h}^{m-1}\right\|_{L^{4}}^{2}+\alpha_{0}^{2}\right) .
\end{aligned}
$$

Thus, taking into account the inductive hypothesis (5.1), from (5.2) and (5.3) we arrive at

$$
\left\|n_{h}^{m}\right\|_{L^{2}}^{2}-\left\|n_{h}^{m-1}\right\|_{L^{2}}^{2}+D_{n} \Delta t\left\|n_{h}^{m}\right\|_{H^{1}}^{2}-\frac{D_{n} \Delta t}{2}\left\|n_{h}^{m-1}\right\|_{H^{1}}^{2} \leq C \frac{\chi^{4}}{D_{n}^{3}} \Delta t\left\|n_{h}^{m-1}\right\|_{L^{2}}^{2}+C \frac{\chi^{2}}{D_{n}} \Delta t \alpha_{0}^{2} .
$$

Then, summing in (5.4) from $m=1$ to $m=r$, we arrive at

$$
\begin{aligned}
& \left\|n_{h}^{r}\right\|_{L^{2}}^{2}+\frac{D_{n}}{2} \Delta t \sum_{m=1}^{r}\left\|n_{h}^{m}\right\|_{H^{1}}^{2}+\frac{D_{n}}{2} \Delta t\left\|n_{h}^{r}\right\|_{H^{1}}^{2} \\
& \quad \leq\left\|n_{h}^{0}\right\|_{L^{2}}^{2}+\frac{D_{n} \Delta t}{2}\left\|n_{h}^{0}\right\|_{H^{1}}^{2}+C \frac{\chi^{4}}{D_{n}^{3}} \Delta t \sum_{m=1}^{r}\left\|n_{h}^{m-1}\right\|_{L^{2}}^{2}+C \frac{\chi^{2}}{D_{n}} \Delta t \sum_{m=1}^{r} \alpha_{0}^{2}
\end{aligned}
$$

Therefore, applying Lemma 5.1 to (5.5) we deduce

$$
\left\|n_{h}^{r}\right\|_{L^{2}}^{2}+\Delta t \sum_{m=1}^{r}\left\|n_{h}^{m}\right\|_{H^{1}}^{2} \leq C, \quad \forall m \geq 1,
$$

where the constant $C>0$ depends on $\left(\chi, D_{n}, \alpha_{0}, T, n_{0}\right)$, but is independent of $(\Delta t, h)$ and $r$; thus we conclude the proof.

Lemma 5.3 (Uniform estimates for $c_{h}^{m}$ ). Assume the inductive hypothesis (5.1). Then, $c_{h}^{m}$ is bounded in $l^{\infty}\left(L^{2}\right) \cap l^{2}\left(H^{1}\right)$.

Proof. Testing $(3.15)_{c}$ by $\bar{c}=2 \Delta t c_{h}^{m}$, using the equality $(a-b, 2 a)=|a|^{2}-|b|^{2}+|a-b|^{2}$ and taking into account the property (3.14), we obtain

$$
\left\|c_{h}^{m}\right\|_{L^{2}}^{2}-\left\|c_{h}^{m-1}\right\|_{L^{2}}^{2}+\left\|c_{h}^{m}-c_{h}^{m-1}\right\|_{L^{2}}^{2}+2 D_{c} \Delta t\left\|\nabla c_{h}^{m}\right\|_{L^{2}}^{2}=-2 \gamma \Delta t\left(\left(n_{h}^{m-1}+\alpha_{0}\right) c_{h}^{m-1}, c_{h}^{m}\right) .
$$

Using the Hölder and Young inequalities, we have

$$
2 \gamma \Delta t\left(\left(n_{h}^{m-1}+\alpha_{0}\right) c_{h}^{m-1}, c_{h}^{m}\right) \leq 2 \gamma \Delta t\left\|n_{h}^{m-1}+\alpha_{0}\right\|_{L^{4}}\left\|c_{h}^{m-1}\right\|_{L^{2}}\left\|c_{h}^{m}\right\|_{L^{4}}
$$




$$
\begin{aligned}
\leq & C \gamma \Delta t\left\|n_{h}^{m-1}+\alpha_{0}\right\|_{L^{4}}\left\|c_{h}^{m-1}\right\|_{L^{2}}\left(\left\|\nabla c_{h}^{m}\right\|_{L^{2}}+\left\|c_{h}^{m}\right\|_{L^{2}}\right) \\
\leq & D_{c} \Delta t\left\|\nabla c_{h}^{m}\right\|_{L^{2}}^{2}+\frac{1}{2}\left\|c_{h}^{m}-c_{h}^{m-1}\right\|_{L^{2}}^{2}+\Delta t\left\|c_{h}^{m-1}\right\|_{L^{2}}^{2} \\
& +C \gamma^{2} \Delta t\left[\frac{1}{D_{c}}+\Delta t+1\right]\left\|n_{h}^{m-1}+\alpha_{0}\right\|_{L^{4}}^{2}\left\|c_{h}^{m-1}\right\|_{L^{2}}^{2} .
\end{aligned}
$$

Therefore, from (5.6) and (5.7), we arrive at

$$
\begin{aligned}
& \left\|c_{h}^{m}\right\|_{L^{2}}^{2}-\left\|c_{h}^{m-1}\right\|_{L^{2}}^{2}+\frac{1}{2}\left\|c_{h}^{m}-c_{h}^{m-1}\right\|_{L^{2}}^{2}+D_{c} \Delta t\left\|\nabla c_{h}^{m}\right\|_{L^{2}}^{2} \\
& \quad \leq C \gamma^{2} \Delta t\left[\frac{1}{D_{c}}+\Delta t+1\right]\left\|n_{h}^{m-1}+\alpha_{0}\right\|_{L^{4}}^{2}\left\|c_{h}^{m-1}\right\|_{L^{2}}^{2}+\Delta t\left\|c_{h}^{m-1}\right\|_{L^{2}}^{2} .
\end{aligned}
$$

Then, summing in (5.8) from $m=1$ to $m=r$, applying Lemma 5.1 and taking into account that $n_{h}^{m}$ is bounded in $l^{2}\left(H^{1}\right)$ (see Lem. 5.2), we arrive at

$$
\left\|c_{h}^{r}\right\|_{L^{2}}^{2}+\Delta t \sum_{m=1}^{r}\left\|\nabla c_{h}^{m}\right\|_{L^{2}}^{2} \leq C, \quad \forall m \geq 1,
$$

the constant $C>0$ depends on $\left(\chi, D_{c}, D_{n}, \gamma, \alpha_{0}, T, n_{0}, c_{0}\right)$, but is independent of $(\Delta t, h)$ and $r$.

Remark 5.4. Notice that estimate (5.3) is independent of the dimension. In fact, in the three-dimensional case, one can bound the first inequality in (5.3) with $\left\|n_{h}^{m}\right\|_{L^{3}}$ instead of $\left\|n_{h}^{m}\right\|_{L^{4}}$ and $\left\|\boldsymbol{\sigma}_{h}^{m}\right\|_{L^{6}}$ instead of $\left\|\boldsymbol{\sigma}_{h}^{m}\right\|_{L^{4}}$, and using the 3D interpolation inequality (2.6), (5.3) remains true; consequently, (5.4) also holds. Therefore, Lemmas 5.2 and 5.3 are true in both dimensions two and three, only by assuming the inductive hypothesis (5.1).

\subsection{Error estimates}

The aim of this subsection is to obtain optimal error estimates for any solution $\left[n_{h}^{m}, c_{h}^{m}, \boldsymbol{\sigma}_{h}^{m}, \mathbf{u}_{h}^{m}, \pi_{h}^{m}\right]$ of the scheme (3.15), with respect to a sufficiently regular solution $[n, c, \boldsymbol{\sigma}, \mathbf{u}, \pi]$ of (2.13) in the two dimensional case. For that we proceed recursively, bounding the error estimates at time $t^{m}$ by quantities depending only on norms of a sufficiently regular solution and preceeding errors at time $t^{m-1}$. We start by introducing the following notations for the errors at $t=t_{m}: e_{n}^{m}=n^{m}-n_{h}^{m}, e_{c}^{m}=c^{m}-c_{h}^{m}, e_{\boldsymbol{\sigma}}^{m}=\boldsymbol{\sigma}^{m}-\boldsymbol{\sigma}_{h}^{m}, e_{\mathbf{u}}^{m}=\mathbf{u}^{m}-\mathbf{u}_{h}^{m}$ and $e_{\pi}^{m}=\pi^{m}-\pi_{h}^{m}$, where $w^{m}$ denotes, in general, the value of $w$ at time $t_{m}$. Then, subtracting the scheme (3.15) to (2.13) at $t=t_{m}$, we obtain that $\left[e_{n}^{m}, e_{c}^{m}, e_{\boldsymbol{\sigma}}^{m}, e_{\mathbf{u}}^{m}, e_{\pi}^{m}\right]$ satisfies

$$
\begin{aligned}
\left(\delta_{t} e_{n}^{m}, \bar{n}\right) & +D_{n}\left(\nabla e_{n}^{m}, \nabla \bar{n}\right)+A\left(\mathbf{u}^{m}-\mathbf{u}^{m-1}, n^{m}, \bar{n}\right)+A\left(\mathbf{u}_{h}^{m-1}, e_{n}^{m}, \bar{n}\right)+A\left(e_{\mathbf{u}}^{m-1}, n^{m}, \bar{n}\right)=\left(\omega_{n}^{m}, \bar{n}\right) \\
& +\chi\left(\left(n^{m-1}+\alpha_{0}\right)\left(\boldsymbol{\sigma}^{m}-\boldsymbol{\sigma}^{m-1}\right)+\left(n^{m}-n^{m-1}\right) \boldsymbol{\sigma}^{m}+\left(n^{m-1}+\alpha_{0}\right) e_{\boldsymbol{\sigma}}^{m-1}+e_{n}^{m-1} \boldsymbol{\sigma}_{h}^{m-1}, \nabla \bar{n}\right), \\
\left(\delta_{t} e_{\boldsymbol{\sigma}}^{m}, \overline{\boldsymbol{\sigma}}\right) & +D_{c}\left(\nabla \cdot e_{\boldsymbol{\sigma}}^{m}, \nabla \cdot \overline{\boldsymbol{\sigma}}\right)+D_{c}\left(\operatorname{rot} e_{\boldsymbol{\sigma}}^{m}, \operatorname{rot} \overline{\boldsymbol{\sigma}}\right)=\left(\omega_{\boldsymbol{\sigma}}^{m}, \overline{\boldsymbol{\sigma}}\right)+\left(\left(\mathbf{u}^{m}-\mathbf{u}^{m-1}\right) \cdot \boldsymbol{\sigma}^{m}, \nabla \cdot \overline{\boldsymbol{\sigma}}\right) \\
& +\left(\mathbf{u}^{m-1}\left(\boldsymbol{\sigma}^{m}-\boldsymbol{\sigma}^{m-1}\right)+e_{\mathbf{u}}^{m-1} \boldsymbol{\sigma}^{m-1}+\mathbf{u}_{h}^{m-1} e_{\boldsymbol{\sigma}}^{m-1}+\gamma\left(n^{m}-n^{m-1}\right) c^{m}, \nabla \cdot \overline{\boldsymbol{\sigma}}\right) \\
& +\gamma\left(\left(n^{m-1}+\alpha_{0}\right)\left(c^{m}-c^{m-1}\right)+e_{n}^{m-1} c_{h}^{m-1}+\left(n^{m-1}+\alpha_{0}\right) e_{c}^{m-1}, \nabla \cdot \overline{\boldsymbol{\sigma}}\right), \\
\left(\delta_{t} e_{c}^{m}, \bar{c}\right) & +D_{c}\left(\nabla e_{c}^{m}, \nabla \bar{c}\right)+A\left(\mathbf{u}^{m}-\mathbf{u}^{m-1}, c^{m}, \bar{c}\right)+A\left(\mathbf{u}_{h}^{m-1}, e_{c}^{m}, \bar{c}\right)+A\left(e_{\mathbf{u}}^{m-1}, c^{m}, \bar{c}\right)=\left(\omega_{c}^{m}, \bar{c}\right) \\
& -\gamma\left(\left(n^{m}-n^{m-1}\right) c^{m}+\left(n^{m-1}+\alpha_{0}\right)\left(c^{m}-c^{m-1}\right)+e_{n}^{m-1} c^{m-1}+\left(n_{h}^{m-1}+\alpha_{0}\right) e_{c}^{m-1}, \bar{c}\right), \\
\left(\delta_{t} e_{\mathbf{u}}^{m}, \overline{\mathbf{u}}\right) & +\frac{D_{\mathbf{u}}}{\rho}\left(\nabla e_{\mathbf{u}}^{m}, \nabla \overline{\mathbf{u}}\right)=\left(\omega_{\mathbf{u}}^{m}, \overline{\mathbf{u}}\right)-B\left(\mathbf{u}^{m}-\mathbf{u}^{m-1}, \mathbf{u}^{m}, \overline{\mathbf{u}}\right)-B\left(e_{\mathbf{u}}^{m-1}, \mathbf{u}^{m}, \overline{\mathbf{u}}\right) \\
& -B\left(\mathbf{u}_{h}^{m-1}, e_{\mathbf{u}}^{m}, \overline{\mathbf{u}}\right)+\frac{1}{\rho}\left(e_{\pi}^{m}, \nabla \cdot \overline{\mathbf{u}}\right)+\frac{1}{\rho}\left(\left(n^{m}-n^{m-1}\right) \nabla \phi, \overline{\mathbf{u}}\right)+\frac{1}{\rho}\left(e_{n}^{m-1} \nabla \phi, \overline{\mathbf{u}}\right), \\
( & \left.\cdot e_{\mathbf{u}}^{m}, \bar{\pi}\right)=0,
\end{aligned}
$$


for all $[\bar{n}, \bar{c}, \overline{\boldsymbol{\sigma}}, \overline{\mathbf{u}}, \bar{\pi}] \in \mathcal{X}_{n} \times \mathcal{X}_{c} \times \mathcal{X}_{\boldsymbol{\sigma}} \times \mathcal{X}_{\mathbf{u}} \times \mathcal{X}_{\pi}$, where $\omega_{n}^{m}, \omega_{c}^{m}, \omega_{\boldsymbol{\sigma}}^{m}, \omega_{\mathbf{u}}^{m}$ are the consistency errors associated to the scheme (3.15), that is, $\omega_{n}^{m}=\delta_{t} n^{m}-\left(n_{t}\right)^{m}$ and so on.

Considering the interpolation operators $\mathbb{P}_{n}, \mathbb{P}_{c}, \mathbb{P}_{\boldsymbol{\sigma}}, \mathbb{P}_{\mathbf{u}}$ and $\mathbb{P}_{\pi}$ defined in (3.5) and (3.2), we decompose the total errors $e_{n}^{m}, e_{c}^{m}, e_{\boldsymbol{\sigma}}^{m}, e_{\mathbf{u}}^{m}$ and $e_{\pi}^{m}$ as follows:

$$
\begin{aligned}
& e_{n}^{m}=\left(n^{m}-\mathbb{P}_{n} n^{m}\right)+\left(\mathbb{P}_{n} n^{m}-n_{h}^{m}\right)=\theta_{n}^{m}+\xi_{n}^{m}, \\
& e_{c}^{m}=\left(c^{m}-\mathbb{P}_{c} c^{m}\right)+\left(\mathbb{P}_{c} c^{m}-c_{h}^{m}\right)=\theta_{c}^{m}+\xi_{c}^{m}, \\
& e_{\boldsymbol{\sigma}}^{m}=\left(\boldsymbol{\sigma}^{m}-\mathbb{P}_{\boldsymbol{\sigma}} \boldsymbol{\sigma}^{m}\right)+\left(\mathbb{P}_{\boldsymbol{\sigma}} \boldsymbol{\sigma}^{m}-\boldsymbol{\sigma}_{h}^{m}\right)=\theta_{\boldsymbol{\sigma}}^{m}+\xi_{\boldsymbol{\sigma}}^{m}, \\
& e_{\mathbf{u}}^{m}=\left(\mathbf{u}^{m}-\mathbb{P}_{\mathbf{u}} \mathbf{u}^{m}\right)+\left(\mathbb{P}_{\mathbf{u}} \mathbf{u}^{m}-\mathbf{u}_{h}^{m}\right)=\theta_{\mathbf{u}}^{m}+\xi_{\mathbf{u}}^{m}, \\
& e_{\pi}^{m}=\left(\pi^{m}-\mathbb{P}_{\pi} \pi^{m}\right)+\left(\mathbb{P}_{\pi} \pi^{m}-\pi_{h}^{m}\right)=\theta_{\pi}^{m}+\xi_{\pi}^{m},
\end{aligned}
$$

where, in general, $\theta_{a}^{m}$ and $\xi_{a}^{m}$ denote the interpolation and discrete errors with respect to the $a$ variable, respectively. Thus, the aim of this section is to prove the following result:

Theorem 5.5. Assuming (5.1), the following estimates for the discrete errors hold

$$
\begin{aligned}
\left\|\left[\xi_{n}^{m}, \xi_{c}^{m}, \xi_{\mathbf{u}}^{m}, \xi_{\boldsymbol{\sigma}}^{m}\right]\right\|_{l^{\infty}\left(L^{2}\right) \cap l^{2}\left(H^{1}\right)} & \leq C(T)\left(\Delta t+\max \left\{h^{r_{1}+1}, h^{r_{2}+1}, h^{r_{3}+1}, h^{r+1}\right\}\right), \\
\left\|\xi_{\mathbf{u}}^{m}\right\|_{l^{\infty}\left(H^{1}\right) \cap l^{2}\left(W^{1,6}\right)}+\left\|\xi_{\pi}^{m}\right\|_{l^{2}\left(L^{6}\right)} & \leq C(T)\left(\Delta t+\max \left\{h^{r_{1}+1}, h^{r_{2}+1}, h^{r_{3}+1}, h^{r}\right\}\right),
\end{aligned}
$$

where the constant $C(T)>0$ is independent of $m, \Delta t$ and $h$.

From decompositions (5.14)-(5.18), Theorem 5.5 and interpolation errors (3.3) and (3.6), one can deduce:

Corollary 5.6. Under the assumptions of Theorem 5.5, the following estimates for the total errors hold

$$
\begin{aligned}
\left\|\left[e_{n}^{m}, e_{c}^{m}, e_{\mathbf{u}}^{m}, e_{\boldsymbol{\sigma}}^{m}\right]\right\|_{l^{\infty}\left(L^{2}\right)} & \leq C(T)\left(\Delta t+\max \left\{h^{r_{1}+1}, h^{r_{2}+1}, h^{r_{3}+1}, h^{r+1}\right\}\right), \\
\left\|\left[e_{n}^{m}, e_{c}^{m}, e_{\mathbf{u}}^{m}, e_{\boldsymbol{\sigma}}^{m}\right]\right\|_{l^{2}\left(H^{1}\right)} & \leq C(T)\left(\Delta t+\max \left\{h^{r_{1}}, h^{r_{2}}, h^{r_{3}}, h^{r}\right\}\right), \\
\left\|e_{\mathbf{u}}^{m}\right\|_{l^{\infty}\left(H^{1}\right)} & \leq C(T)\left(\Delta t+\max \left\{h^{r_{1}+1}, h^{r_{2}+1}, h^{r_{3}+1}, h^{r}\right\}\right),
\end{aligned}
$$

where the constant $C(T)>0$ is independent of $m, \Delta t$ and $h$.

Also, the following result is obtained directly as a consequence of Theorem 5.5.

Corollary 5.7. Let $\left[n_{h}^{m}, c_{h}^{m}, \boldsymbol{\sigma}_{h}^{m}, \mathbf{u}_{h}^{m}, \pi_{h}^{m}\right]$ be any solution of the scheme (3.15) and consider a sufficiently regular solution $[n, c, \boldsymbol{\sigma}, \mathbf{u}, \pi]$ of (2.13). Then, $\left[n_{h}^{m}, c_{h}^{m}, \boldsymbol{\sigma}_{h}^{m}, \mathbf{u}_{h}^{m}, \pi_{h}^{m}\right]$ converges to $[n, c, \boldsymbol{\sigma}, \mathbf{u}, \pi]$ in $l^{\infty}\left(L^{2}\right), l^{2}\left(H^{1}\right)$-norms, when the parameters $\Delta t$ and $h$ go to 0 .

Remark 5.8. From (5.19) and (5.20), in particular we deduce that $\left\|\left[\mathbf{u}_{h}^{m}, \boldsymbol{\sigma}_{h}^{m}\right]\right\|_{l^{\infty}\left(L^{2}\right) \cap l^{2}\left(H^{1}\right)} \leq C(T)$ and $\left\|\left[\mathbf{u}_{h}^{m}, \pi_{h}^{m}\right]\right\|_{l^{\infty}\left(H^{1}\right) \times l^{2}\left(L^{6}\right)} \leq C(T)$, for all $m=1, \ldots, N$.

\subsubsection{Preliminary error estimates}

Now, before proving Theorem 5.5, we will obtain some bounds for the discrete errors of the cell density $n$, the chemical concentration $c$, the flux $\boldsymbol{\sigma}$ and the velocity $\mathbf{u}$.

(1) Error estimate for the cell density $n$

Taking into account $(3.5)_{1}$, from (5.9), (5.14), (5.16) and (5.17) we have

$$
\begin{aligned}
\left(\delta_{t} \xi_{n}^{m}, \bar{n}\right) & +D_{n}\left(\nabla \xi_{n}^{m}, \nabla \bar{n}\right)+A\left(\mathbf{u}_{h}^{m-1}, \xi_{n}^{m}, \bar{n}\right)=\left(\omega_{n}^{m}, \bar{n}\right)-\left(\delta_{t} \theta_{n}^{m}, \bar{n}\right)-A\left(\mathbf{u}_{h}^{m-1}, \theta_{n}^{m}, \bar{n}\right) \\
& -A\left(\mathbf{u}^{m}-\mathbf{u}^{m-1}, n^{m}, \bar{n}\right)-A\left(\left(\xi_{\mathbf{u}}^{m-1}+\theta_{\mathbf{u}}^{m-1}\right), n^{m}, \bar{n}\right)+\chi\left(\left(n^{m-1}+\alpha_{0}\right)\left(\boldsymbol{\sigma}^{m}-\boldsymbol{\sigma}^{m-1}\right), \nabla \bar{n}\right) \\
& +\chi\left(\left(n^{m}-n^{m-1}\right) \boldsymbol{\sigma}^{m}+\left(n^{m-1}+\alpha_{0}\right)\left(\xi_{\boldsymbol{\sigma}}^{m-1}+\theta_{\boldsymbol{\sigma}}^{m-1}\right)+\left(\xi_{n}^{m-1}+\theta_{n}^{m-1}\right) \boldsymbol{\sigma}_{h}^{m-1}, \nabla \bar{n}\right),
\end{aligned}
$$


for all $\bar{n} \in \mathcal{X}_{n}$. Taking $\bar{n}=\xi_{n}^{m}$ in (5.21), using (3.14) and taking into account that $\int_{\Omega} \xi_{n}^{m}=0$ (since $\xi_{n}^{m} \in \mathcal{X}_{n}$ ), we get

$$
\begin{aligned}
\frac{1}{2} \delta_{t}\left\|\xi_{n}^{m}\right\|_{L^{2}}^{2} & +\frac{\Delta t}{2}\left\|\delta_{t} \xi_{n}^{m}\right\|_{L^{2}}^{2}+D_{n}\left\|\xi_{n}^{m}\right\|_{H^{1}}^{2}=\left(\omega_{n}^{m}, \xi_{n}^{m}\right)-\left(\delta_{t} \theta_{n}^{m}, \xi_{n}^{m}\right)-A\left(\mathbf{u}_{h}^{m-1}, \theta_{n}^{m}, \xi_{n}^{m}\right) \\
& -A\left(\mathbf{u}^{m}-\mathbf{u}^{m-1}, n^{m}, \xi_{n}^{m}\right)-A\left(\left(\xi_{\mathbf{u}}^{m-1}+\theta_{\mathbf{u}}^{m-1}\right), n^{m}, \xi_{n}^{m}\right)+\chi\left(\left(n^{m-1}+\alpha_{0}\right)\left(\boldsymbol{\sigma}^{m}-\boldsymbol{\sigma}^{m-1}\right), \nabla \xi_{n}^{m}\right) \\
& +\chi\left(\left(n^{m}-n^{m-1}\right) \boldsymbol{\sigma}^{m}, \nabla \xi_{n}^{m}\right)+\chi\left(\left(n^{m-1}+\alpha_{0}\right)\left(\xi_{\boldsymbol{\sigma}}^{m-1}+\theta_{\boldsymbol{\sigma}}^{m-1}\right), \nabla \xi_{n}^{m}\right) \\
& +\chi\left(\left(\xi_{n}^{m-1}+\theta_{n}^{m-1}\right) \boldsymbol{\sigma}_{h}^{m-1}, \nabla \xi_{n}^{m}\right)=\sum_{k=1}^{9} I_{k} .
\end{aligned}
$$

Then, we control the terms on the right hand side of (5.22) as follows: First, using the Hölder and Young inequalities, and (3.6) we get

$$
I_{1} \leq \frac{D_{n}}{12}\left\|\xi_{n}^{m}\right\|_{H^{1}}^{2}+\frac{C}{D_{n}}\left\|\omega_{n}^{m}\right\|_{\left(H^{1}\right)^{\prime}}^{2} \leq \frac{D_{n}}{12}\left\|\xi_{n}^{m}\right\|_{H^{1}}^{2}+\frac{C \Delta t}{D_{n}} \int_{t_{m-1}}^{t_{m}}\left\|n_{t t}(t)\right\|_{\left(H^{1}\right)^{\prime}}^{2} \mathrm{~d} t
$$

and

$$
\begin{aligned}
I_{2} & \leq\left\|\xi_{n}^{m}\right\|_{L^{2}}\left\|\left(\mathcal{I}-\mathbb{P}_{n}\right) \delta_{t} n^{m}\right\|_{L^{2}} \leq \frac{D_{n}}{12}\left\|\xi_{n}^{m}\right\|_{L^{2}}^{2}+\frac{C h^{2\left(r_{1}+1\right)}}{D_{n}}\left\|\delta_{t} n^{m}\right\|_{H^{r_{1}+1}}^{2} \\
& \leq \frac{D_{n}}{12}\left\|\xi_{n}^{m}\right\|_{L^{2}}^{2}+\frac{C h^{2\left(r_{1}+1\right)}}{D_{n} \Delta t} \int_{t_{m-1}}^{t_{m}}\left\|n_{t}\right\|_{H^{r_{1}+1}}^{2} \mathrm{~d} t .
\end{aligned}
$$

From the definition of the skew-symmetric trilinear form (3.10), the Hölder and Young inequalities, (2.4), (3.4), (3.6) and (3.8) we obtain

$$
\begin{aligned}
I_{3} & =A\left(\xi_{\mathbf{u}}^{m-1}, \theta_{n}^{m}, \xi_{n}^{m}\right)-A\left(\mathbb{P}_{\mathbf{u}} \mathbf{u}^{m-1}, \theta_{n}^{m}, \xi_{n}^{m}\right) \\
& \leq\left\|\xi_{\mathbf{u}}^{m-1}\right\|_{L^{2}}\left\|\theta_{n}^{m}\right\|_{L^{\infty} \cap W^{1,3}}\left\|\xi_{n}^{m}\right\|_{H^{1}}+\left\|\mathbb{P}_{\mathbf{u}} \mathbf{u}^{m-1}\right\|_{L^{\infty} \cap W^{1,3}}\left\|\theta_{n}^{m}\right\|_{L^{2}}\left\|\xi_{n}^{m}\right\|_{H^{1}} \\
& \leq \frac{D_{n}}{12}\left\|\xi_{n}^{m}\right\|_{H^{1}}^{2}+\frac{C}{D_{n}}\left\|n^{m}\right\|_{H^{2}}^{2}\left\|\xi_{\mathbf{u}}^{m-1}\right\|_{L^{2}}^{2}+\frac{C}{D_{n}} h^{2\left(r_{1}+1\right)}\left\|\left[\mathbf{u}^{m-1}, \pi^{m-1}\right]\right\|_{H^{2} \times H^{1}}^{2}\left\|n^{m}\right\|_{H^{r_{1}+1}}^{2} .
\end{aligned}
$$

Now, using the Hölder and Young inequalities, (2.4) and (3.3), we have

$$
\begin{aligned}
I_{4}+I_{5} \leq & \left(\left\|\mathbf{u}^{m}-\mathbf{u}^{m-1}\right\|_{L^{2}}+\left\|\xi_{\mathbf{u}}^{m-1}\right\|_{L^{2}}+\left\|\theta_{\mathbf{u}}^{m-1}\right\|_{L^{2}}\right)\left\|n^{m}\right\|_{L^{\infty} \cap W^{1,3}}\left\|\xi_{n}^{m}\right\|_{H^{1}} \\
\leq & \frac{D_{n}}{12}\left\|\xi_{n}^{m}\right\|_{H^{1}}^{2}+\frac{C}{D_{n}}\left\|\mathbf{u}^{m}-\mathbf{u}^{m-1}\right\|_{L^{2}}^{2}\left\|n^{m}\right\|_{H^{2}}^{2} \\
& +\frac{C}{D_{n}}\left(h^{2(r+1)}\left\|\left[\mathbf{u}^{m-1}, \pi^{m-1}\right]\right\|_{H^{r+1} \times H^{r}}^{2}+\left\|\xi_{\mathbf{u}}^{m-1}\right\|_{L^{2}}^{2}\right)\left\|n^{m}\right\|_{H^{2}}^{2}
\end{aligned}
$$

Also, from the Hölder and Young inequalities, and (3.6) we get

$$
\begin{aligned}
\sum_{k=6}^{8} I_{k} \leq & \chi\left\|\left[\boldsymbol{\sigma}^{m}-\boldsymbol{\sigma}^{m-1}, n^{m}-n^{m-1}, \xi_{\boldsymbol{\sigma}}^{m-1}, \theta_{\boldsymbol{\sigma}}^{m-1}\right]\right\|_{L^{2}}\left\|\left[n^{m-1}+\alpha_{0}, \boldsymbol{\sigma}^{m}\right]\right\|_{L^{\infty}}\left\|\xi_{n}^{m}\right\|_{H^{1}} \\
\leq & \frac{D_{n}}{12}\left\|\xi_{n}^{m}\right\|_{H^{1}}^{2}+\frac{C \chi^{2}}{D_{n}}\left(\left\|\left[\boldsymbol{\sigma}^{m}-\boldsymbol{\sigma}^{m-1}, n^{m}-n^{m-1}\right]\right\|_{L^{2}}^{2}+\left\|\xi_{\boldsymbol{\sigma}}^{m-1}\right\|_{L^{2}}^{2}\right)\left\|\left[n^{m-1}+\alpha_{0}, \boldsymbol{\sigma}^{m}\right]\right\|_{L^{\infty}}^{2} \\
& +\frac{C \chi^{2}}{D_{n}} h^{2\left(r_{3}+1\right)}\left\|\boldsymbol{\sigma}^{m-1}\right\|_{H^{r_{3}+1}}^{2}\left\|\left[n^{m-1}+\alpha_{0}, \boldsymbol{\sigma}^{m}\right]\right\|_{L^{\infty}}^{2} .
\end{aligned}
$$


Finally, from the Hölder and Young inequalities, (2.4), (3.6), (3.8), the 2D interpolation inequality (2.5) and the inductive hypothesis (5.1), we can bound

$$
\begin{aligned}
I_{9}= & \chi\left(\xi_{n}^{m-1} \boldsymbol{\sigma}_{h}^{m-1}, \nabla \xi_{n}^{m}\right)-\chi\left(\theta_{n}^{m-1} \xi_{\boldsymbol{\sigma}}^{m-1}, \nabla \xi_{n}^{m}\right)+\chi\left(\theta_{n}^{m-1} \mathbb{P}_{\boldsymbol{\sigma}} \boldsymbol{\sigma}^{m-1}, \nabla \xi_{n}^{m}\right) \\
\leq & \chi C\left(\left\|\xi_{n}^{m-1}\right\|_{L^{2}}^{1 / 2}\left\|\xi_{n}^{m-1}\right\|_{H^{1}}^{1 / 2}\left\|\boldsymbol{\sigma}_{h}^{m-1}\right\|_{L^{4}}+\left\|\xi_{\boldsymbol{\sigma}}^{m-1}\right\|_{L^{2}}\left\|\theta_{n}^{m-1}\right\|_{L^{\infty}}+\left\|\mathbb{P}_{\boldsymbol{\sigma}} \boldsymbol{\sigma}^{m-1}\right\|_{L^{\infty}}\left\|\theta_{n}^{m-1}\right\|_{L^{2}}\right)\left\|\xi_{n}^{m}\right\|_{H^{1}} \\
\leq & \frac{D_{n}}{12}\left\|\xi_{n}^{m}\right\|_{H^{1}}^{2}+\frac{D_{n}}{8}\left\|\xi_{n}^{m-1}\right\|_{H^{1}}^{2}+\frac{C \chi^{4}}{D_{n}^{3}}\left\|\xi_{n}^{m-1}\right\|_{L^{2}}^{2} \\
& +\frac{C \chi^{2}}{D_{n}}\left\|n^{m-1}\right\|_{H^{2}}^{2}\left\|\xi_{\boldsymbol{\sigma}}^{m-1}\right\|_{L^{2}}^{2}+\frac{C \chi^{2}}{D_{n}} h^{2\left(r_{1}+1\right)}\left\|\boldsymbol{\sigma}^{m-1}\right\|_{H^{2}}^{2}\left\|n^{m-1}\right\|_{H^{r_{1}+1}}^{2} .
\end{aligned}
$$

Therefore, from (5.22) to (5.28), we arrive at

$$
\begin{aligned}
\frac{1}{2} \delta_{t}\left\|\xi_{n}^{m}\right\|_{L^{2}}^{2} & +\frac{\Delta t}{2}\left\|\delta_{t} \xi_{n}^{m}\right\|_{L^{2}}^{2}+\frac{D_{n}}{2}\left\|\xi_{n}^{m}\right\|_{H^{1}}^{2}-\frac{D_{n}}{8}\left\|\xi_{n}^{m-1}\right\|_{H^{1}}^{2} \leq \frac{C h^{2\left(r_{1}+1\right)}}{D_{n} \Delta t} \int_{t_{m-1}}^{t_{m}}\left\|n_{t}\right\|_{H^{r_{1}+1}}^{2} \mathrm{~d} t \\
& +\frac{C \Delta t}{D_{n}} \int_{t_{m-1}}^{t_{m}}\left\|n_{t t}(t)\right\|_{\left(H^{1}\right)^{\prime}}^{2} \mathrm{~d} t+\frac{C}{D_{n}}\left\|n^{m}\right\|_{H^{2}}^{2}\left\|\xi_{\mathbf{u}}^{m-1}\right\|_{L^{2}}^{2} \\
& +\frac{C}{D_{n}} h^{2\left(r_{1}+1\right)}\left\|\left[\mathbf{u}^{m-1}, \pi^{m-1}\right]\right\|_{H^{2} \times H^{1}}^{2}\left\|n^{m}\right\|_{H^{r_{1}+1}}^{2} \\
& +\frac{C}{D_{n}}\left\|\mathbf{u}^{m}-\mathbf{u}^{m-1}\right\|_{L^{2}}^{2}\left\|n^{m}\right\|_{H^{2}}^{2}+\frac{C}{D_{n}} h^{2(r+1)}\left\|\left[\mathbf{u}^{m-1}, \pi^{m-1}\right]\right\|_{H^{r+1} \times H^{r}}^{2}\left\|n^{m}\right\|_{H^{2}}^{2} \\
& \left.+\frac{C \chi^{2}}{D_{n}}\left(\left\|\left[\boldsymbol{\sigma}^{m}-\boldsymbol{\sigma}^{m-1}, n^{m}-n^{m-1}\right]\right\|_{L^{2}}^{2}+\left\|\xi_{\boldsymbol{\sigma}}^{m-1}\right\|_{L^{2}}^{2}+h^{2\left(r_{3}+1\right)}\left\|\boldsymbol{\sigma}^{m-1}\right\|_{H^{r_{3}+1}}^{2}\right) \| n^{m-1}+\alpha_{0}, \boldsymbol{\sigma}^{m}\right] \|_{L^{\infty}}^{2} \\
& +\frac{C \chi^{4}}{D_{n}^{3}}\left\|\xi_{n}^{m-1}\right\|_{L^{2}}^{2}+\frac{C \chi^{2}}{D_{n}}\left\|n^{m-1}\right\|_{H^{2}}^{2}\left\|\xi_{\boldsymbol{\sigma}}^{m-1}\right\|_{L^{2}}^{2}+\frac{C \chi^{2}}{D_{n}} h^{2\left(r_{1}+1\right)}\left\|\boldsymbol{\sigma}^{m-1}\right\|_{H^{2}}^{2}\left\|n^{m-1}\right\|_{H^{r_{1}+1}}^{2} .
\end{aligned}
$$

(2) Error estimate for the chemical concentration $c$

Taking into account $(3.5)_{2}$, from $(5.11),(5.14),(5.15)$ and (5.17), we have

$$
\begin{aligned}
\left(\delta_{t} \xi_{c}^{m}, \bar{c}\right) & +D_{c}\left(\nabla \xi_{c}^{m}, \nabla \bar{c}\right)+A\left(\mathbf{u}_{h}^{m-1}, \xi_{c}^{m}, \bar{c}\right)=\left(\omega_{c}^{m}, \bar{c}\right)-\left(\delta_{t} \theta_{c}^{m}, \bar{c}\right)-A\left(\mathbf{u}^{m}-\mathbf{u}^{m-1}, c^{m}, \bar{c}\right) \\
& -A\left(\mathbf{u}_{h}^{m-1}, \theta_{c}^{m}, \bar{c}\right)-A\left(\left(\xi_{\mathbf{u}}^{m-1}+\theta_{\mathbf{u}}^{m-1}\right), c^{m}, \bar{c}\right)-\gamma\left(\left(n^{m}-n^{m-1}\right) c^{m}+\left(n^{m-1}+\alpha_{0}\right)\left(c^{m}-c^{m-1}\right), \bar{c}\right) \\
& -\gamma\left(\left(\xi_{n}^{m-1}+\theta_{n}^{m-1}\right) c^{m-1}+\left(n_{h}^{m-1}+\alpha_{0}\right)\left(\xi_{c}^{m-1}+\theta_{c}^{m-1}\right), \bar{c}\right)+D_{c}\left(\theta_{c}^{m}, \bar{c}\right),
\end{aligned}
$$

for all $\bar{c} \in \mathcal{X}_{c}$. Taking $\bar{c}=\xi_{c}^{m}$ in (5.30) and using (3.14), we get

$$
\begin{aligned}
\frac{1}{2} \delta_{t}\left\|\xi_{c}^{m}\right\|_{L^{2}}^{2} & +\frac{\Delta t}{2}\left\|\delta_{t} \xi_{c}^{m}\right\|_{L^{2}}^{2}+D_{c}\left\|\nabla \xi_{c}^{m}\right\|_{L^{2}}^{2}=\left(\omega_{c}^{m}, \xi_{c}^{m}\right)-\left(\delta_{t} \theta_{c}^{m}, \xi_{c}^{m}\right)-A\left(\mathbf{u}^{m}-\mathbf{u}^{m-1}, c^{m}, \xi_{c}^{m}\right) \\
& -A\left(\mathbf{u}_{h}^{m-1}, \theta_{c}^{m}, \xi_{c}^{m}\right)-A\left(\left(\xi_{\mathbf{u}}^{m-1}+\theta_{\mathbf{u}}^{m-1}\right), c^{m}, \xi_{c}^{m}\right) \\
& -\gamma\left(\left(n^{m}-n^{m-1}\right) c^{m}+\left(n^{m-1}+\alpha_{0}\right)\left(c^{m}-c^{m-1}\right), \xi_{c}^{m}\right) \\
& -\gamma\left(\left(\xi_{n}^{m-1}+\theta_{n}^{m-1}\right) c^{m-1}, \xi_{c}^{m}\right)-\gamma\left(\left(n_{h}^{m-1}+\alpha_{0}\right)\left(\xi_{c}^{m-1}+\theta_{c}^{m-1}\right), \xi_{c}^{m}\right) \\
& +D_{c}\left(\theta_{c}^{m}, \xi_{c}^{m}\right)=\sum_{k=1}^{9} J_{k} .
\end{aligned}
$$

Then, we control the terms on the right hand side of (5.31) as follows: First, using the Hölder and Young inequalities we get

$$
J_{1} \leq\left(\left\|\xi_{c}^{m}\right\|_{L^{2}}+\left\|\nabla \xi_{c}^{m}\right\|_{L^{2}}\right)\left\|\omega_{c}^{m}\right\|_{\left(H^{1}\right)^{\prime}} \leq\left(\Delta t\left\|\delta_{t} \xi_{c}^{m}\right\|_{L^{2}}+\left\|\xi_{c}^{m-1}\right\|_{L^{2}}+\left\|\nabla \xi_{c}^{m}\right\|_{L^{2}}\right)\left\|\omega_{c}^{m}\right\|_{\left(H^{1}\right)^{\prime}}
$$




$$
\begin{aligned}
& \leq \frac{D_{c}}{8}\left\|\nabla \xi_{c}^{m}\right\|_{L^{2}}^{2}+\frac{\Delta t}{24}\left\|\delta_{t} \xi_{c}^{m}\right\|_{L^{2}}^{2}+\frac{1}{2}\left\|\xi_{c}^{m-1}\right\|_{L^{2}}^{2}+\left(\frac{C}{D_{c}}+C \Delta t+\frac{1}{2}\right)\left\|\omega_{c}^{m}\right\|_{\left(H^{1}\right)^{\prime}}^{2} \\
& \leq \frac{D_{c}}{8}\left\|\nabla \xi_{c}^{m}\right\|_{L^{2}}^{2}+\frac{\Delta t}{24}\left\|\delta_{t} \xi_{c}^{m}\right\|_{L^{2}}^{2}+\frac{1}{2}\left\|\xi_{c}^{m-1}\right\|_{L^{2}}^{2}+\left(\frac{1}{D_{c}}+\Delta t+1\right) C \Delta t \int_{t_{m-1}}^{t_{m}}\left\|c_{t t}(t)\right\|_{\left(H^{1}\right)^{\prime}}^{2} \mathrm{~d} t .
\end{aligned}
$$

Using the Hölder and Young inequalities and (3.6), we obtain

$$
\begin{aligned}
J_{2}+J_{9} & \leq\left\|\xi_{c}^{m}\right\|_{L^{2}}\left\|\left(\mathcal{I}-\mathbb{P}_{c}\right) \delta_{t} c^{m}\right\|_{L^{2}}+D_{c}\left\|\xi_{c}^{m}\right\|_{L^{2}}\left\|\theta_{c}^{m}\right\|_{L^{2}} \\
& \leq\left(\left\|\left(\mathcal{I}-\mathbb{P}_{c}\right) \delta_{t} c^{m}\right\|_{L^{2}}+D_{c}\left\|\theta_{c}^{m}\right\|_{L^{2}}\right)\left(\Delta t\left\|\delta_{t} \xi_{c}^{m}\right\|_{L^{2}}+\left\|\xi_{c}^{m-1}\right\|_{L^{2}}\right) \\
& \leq \frac{\Delta t}{24}\left\|\delta_{t} \xi_{c}^{m}\right\|_{L^{2}}^{2}+\frac{1}{2}\left\|\xi_{c}^{m-1}\right\|_{L^{2}}^{2}+(\Delta t+1) C h^{2\left(r_{2}+1\right)}\left[\left\|\delta_{t} c^{m}\right\|_{H^{r_{2}+1}}^{2}+D_{c}^{2}\left\|c^{m}\right\|_{H^{r_{2}+1}}^{2}\right] \\
& \leq \frac{\Delta t}{24}\left\|\delta_{t} \xi_{c}^{m}\right\|_{L^{2}}^{2}+\frac{1}{2}\left\|\xi_{c}^{m-1}\right\|_{L^{2}}^{2}+(\Delta t+1) C h^{2\left(r_{2}+1\right)}\left[\frac{1}{\Delta t} \int_{t_{m-1}}^{t_{m}}\left\|c_{t}\right\|_{H^{r_{2}+1}}^{2} \mathrm{~d} t+D_{c}^{2}\left\|c^{m}\right\|_{H^{r_{2}+1}}^{2}\right] .
\end{aligned}
$$

From the definition of the skew-symmetric trilinear form (3.10), the Hölder and Young inequalities, (2.4), (3.4), (3.6) and (3.8) we have

$$
\begin{aligned}
& J_{4}=A\left(\xi_{\mathbf{u}}^{m-1}, \theta_{c}^{m}, \xi_{c}^{m}\right)-A\left(\mathbb{P}_{\mathbf{u}} \mathbf{u}^{m-1}, \theta_{c}^{m}, \xi_{c}^{m}\right) \\
& \leq\left\|\xi_{\mathbf{u}}^{m-1}\right\|_{L^{2}}\left\|\theta_{c}^{m}\right\|_{L^{\infty} \cap W^{1,3}}\left\|\xi_{c}^{m}\right\|_{H^{1}}+\left\|\mathbb{P}_{\mathbf{u}} \mathbf{u}^{m-1}\right\|_{L^{\infty} \cap W^{1,3}}\left\|\theta_{c}^{m}\right\|_{L^{2}}\left\|\xi_{c}^{m}\right\|_{H^{1}} \\
& \leq\left(\left\|\xi_{\mathbf{u}}^{m-1}\right\|_{L^{2}}\left\|\theta_{c}^{m}\right\|_{L^{\infty} \cap W^{1,3}}+\left\|\mathbb{P}_{\mathbf{u}} \mathbf{u}^{m-1}\right\|_{L^{\infty} \cap W^{1,3}}\left\|\theta_{c}^{m}\right\|_{L^{2}}\right)\left(\Delta t\left\|\delta_{t} \xi_{c}^{m}\right\|_{L^{2}}+\left\|\xi_{c}^{m-1}\right\|_{L^{2}}+\left\|\nabla \xi_{c}^{m}\right\|_{L^{2}}\right) \\
& \leq \frac{D_{c}}{8}\left\|\nabla \xi_{c}^{m}\right\|_{L^{2}}^{2}+\frac{\Delta t}{24}\left\|\delta_{t} \xi_{c}^{m}\right\|_{L^{2}}^{2}+\frac{1}{2}\left\|\xi_{c}^{m-1}\right\|_{L^{2}}^{2}+\left(\frac{1}{D_{c}}+\Delta t+1\right) C\left\|c^{m}\right\|_{H^{2}}^{2}\left\|\xi_{\mathbf{u}}^{m-1}\right\|_{L^{2}}^{2} \\
& +\left(\frac{1}{D_{c}}+\Delta t+1\right) C h^{2\left(r_{2}+1\right)}\left\|\left[\mathbf{u}^{m-1}, \pi^{m-1}\right]\right\|_{H^{2} \times H^{1}}^{2}\left\|c^{m}\right\|_{H^{r_{2}+1}}^{2} .
\end{aligned}
$$

Also, from the Hölder and Young inequalities, (2.4) and (3.3) we get

$$
\begin{aligned}
J_{3}+J_{5} \leq & \left(\left\|\mathbf{u}^{m}-\mathbf{u}^{m-1}\right\|_{L^{2}}+\left\|\xi_{\mathbf{u}}^{m-1}\right\|_{L^{2}}+\left\|\theta_{\mathbf{u}}^{m-1}\right\|_{L^{2}}\right)\left\|c^{m}\right\|_{L^{\infty} \cap W^{1,3}}\left\|\xi_{c}^{m}\right\|_{H^{1}} \\
\leq & \left(\left\|\mathbf{u}^{m}-\mathbf{u}^{m-1}\right\|_{L^{2}}+\left\|\xi_{\mathbf{u}}^{m-1}\right\|_{L^{2}}+\left\|\theta_{\mathbf{u}}^{m-1}\right\|_{L^{2}}\right)\left\|c^{m}\right\|_{L^{\infty} \cap W^{1,3}}\left(\Delta t\left\|\delta_{t} \xi_{c}^{m}\right\|_{L^{2}}+\left\|\xi_{c}^{m-1}\right\|_{L^{2}}+\left\|\nabla \xi_{c}^{m}\right\|_{L^{2}}\right) \\
\leq & \frac{D_{c}}{8}\left\|\nabla \xi_{c}^{m}\right\|_{L^{2}}^{2}+\frac{\Delta t}{24}\left\|\delta_{t} \xi_{c}^{m}\right\|_{L^{2}}^{2}+\frac{1}{2}\left\|\xi_{c}^{m-1}\right\|_{L^{2}}^{2}+\left(\frac{1}{D_{c}}+\Delta t+1\right) C\left\|\mathbf{u}^{m}-\mathbf{u}^{m-1}\right\|_{L^{2}}^{2}\left\|c^{m}\right\|_{H^{2}}^{2} \\
& +\left(\frac{1}{D_{c}}+\Delta t+1\right) C\left(h^{2(r+1)}\left\|\left[\mathbf{u}^{m-1}, \pi^{m-1}\right]\right\|_{H^{r+1} \times H^{r}}^{2}+\left\|\xi_{\mathbf{u}}^{m-1}\right\|_{L^{2}}^{2}\right)\left\|c^{m}\right\|_{H^{2}}^{2} .
\end{aligned}
$$

Again, from the Hölder and Young inequalities, (2.4) and (3.6) we have

$$
\begin{aligned}
\sum_{k=6}^{7} J_{k} \leq & \gamma\left\|\left[n^{m}-n^{m-1}, c^{m}-c^{m-1}, \xi_{n}^{m-1}, \theta_{n}^{m-1}\right]\right\| L_{L^{2}}\left\|\left[c^{m}, n^{m-1}+\alpha_{0}, c^{m-1}\right]\right\|_{L^{\infty}}\left(\Delta t\left\|\delta_{t} \xi_{c}^{m}\right\|_{L^{2}}+\left\|\xi_{c}^{m-1}\right\|_{L^{2}}\right) \\
\leq & \frac{\Delta t}{24}\left\|\delta_{t} \xi_{c}^{m}\right\|_{L^{2}}^{2}+\frac{1}{2}\left\|\xi_{c}^{m-1}\right\|_{L^{2}}^{2}+(\Delta t+1) \gamma^{2} C\left\|\left[n^{m}-n^{m-1}, c^{m}-c^{m-1}\right]\right\|_{L^{2}}^{2}\left\|\left[c^{m}, n^{m-1}+\alpha_{0}, c^{m-1}\right]\right\|_{H^{2}}^{2} \\
& +(\Delta t+1) \gamma^{2} C\left(h^{2\left(r_{1}+1\right)}\left\|n^{m-1}\right\|_{H^{r_{1}+1}}^{2}+\left\|\xi_{n}^{m-1}\right\|_{L^{2}}^{2}\right)\left\|\left[c^{m}, n^{m-1}+\alpha_{0}, c^{m-1}\right]\right\|_{H^{2}}^{2},
\end{aligned}
$$

and

$$
\begin{aligned}
J_{8} \leq & \gamma\left\|\left[\xi_{c}^{m-1}, \theta_{c}^{m-1}\right]\right\|_{L^{2}}\left\|n_{h}^{m-1}+\alpha_{0}\right\|_{L^{3}}\left(\Delta t\left\|\delta_{t} \xi_{c}^{m}\right\|_{L^{2}}+\left\|\xi_{c}^{m-1}\right\|_{L^{2}}+\left\|\nabla \xi_{c}^{m}\right\|_{L^{2}}\right) \\
\leq & \frac{D_{c}}{8}\left\|\nabla \xi_{c}^{m}\right\|_{L^{2}}^{2}+\frac{\Delta t}{24}\left\|\delta_{t} \xi_{c}^{m}\right\|_{L^{2}}^{2}+\frac{1}{2}\left\|\xi_{c}^{m-1}\right\|_{L^{2}}^{2} \\
& +\left(\frac{1}{D_{c}}+\Delta t+1\right) \gamma^{2} C\left(h^{2\left(r_{2}+1\right)}\left\|c^{m-1}\right\|_{H^{r_{2}+1}}^{2}+\left\|\xi_{c}^{m-1}\right\|_{L^{2}}^{2}\right)\left\|n_{h}^{m-1}+\alpha_{0}\right\|_{L^{3}}^{2} .
\end{aligned}
$$


Therefore, denoting $\gamma_{1}:=\frac{1}{D_{c}}+\Delta t+1$ and $\gamma_{2}:=\Delta t+1$, from (5.31) to (5.37), we arrive at

$$
\begin{aligned}
\frac{1}{2} \delta_{t}\left\|\xi_{c}^{m}\right\|_{L^{2}}^{2} & +\frac{\Delta t}{4}\left\|\delta_{t} \xi_{c}^{m}\right\|_{L^{2}}^{2}+\frac{D_{c}}{2}\left\|\nabla \xi_{c}^{m}\right\|_{L^{2}}^{2} \leq \gamma_{1} C\left(\Delta t \int_{t_{m-1}}^{t_{m}}\left\|c_{t t}(t)\right\|_{\left(H^{1}\right)^{\prime}}^{2} \mathrm{~d} t+\left\|c^{m}\right\|_{H^{2}}^{2}\left\|\xi_{\mathbf{u}}^{m-1}\right\|_{L^{2}}^{2}\right) \\
& +\gamma_{2} C h^{2\left(r_{2}+1\right)}\left[\frac{1}{\Delta t} \int_{t_{m-1}}^{t_{m}}\left\|c_{t}\right\|_{H^{r_{2}+1}}^{2} \mathrm{~d} t+D_{c}^{2}\left\|c^{m}\right\|_{H^{r_{2}+1}}^{2}\right] \\
& +\gamma_{1} C h^{2\left(r_{2}+1\right)}\left\|\left[\mathbf{u}^{m-1}, \pi^{m-1}\right]\right\|_{H^{2} \times H^{1}}^{2}\left\|c^{m}\right\|_{H^{r_{2}+1}}^{2} \\
& +\gamma_{1} C\left\|\mathbf{u}^{m}-\mathbf{u}^{m-1}\right\|_{L^{2}}^{2}\left\|c^{m}\right\|_{H^{2}}^{2}+\gamma_{1} C\left(h^{2(r+1)}\left\|\left[\mathbf{u}^{m-1}, \pi^{m-1}\right]\right\|_{H^{r+1} \times H^{r}}^{2}\right)\left\|c^{m}\right\|_{H^{2}}^{2} \\
& +\gamma_{2} \gamma^{2} C\left(\left\|\left[n^{m}-n^{m-1}, c^{m}-c^{m-1}\right]\right\|_{L^{2}}^{2}+h^{2\left(r_{1}+1\right)}\left\|n^{m-1}\right\|_{H^{r_{1}+1}}^{2}+\left\|\xi_{n}^{m-1}\right\|_{L^{2}}^{2}\right) \\
& \times\left\|\left[c^{m}, n^{m-1}+\alpha_{0}, c^{m-1}\right]\right\|_{H^{2}}^{2} \\
& +\gamma_{1} \gamma^{2} C\left(h^{2\left(r_{2}+1\right)}\left\|c^{m-1}\right\|_{H^{r_{2}+1}}^{2}+\left\|\xi_{c}^{m-1}\right\|_{L^{2}}^{2}\right)\left\|n_{h}^{m-1}+\alpha_{0}\right\|_{L^{3}}^{2}+3\left\|\xi_{c}^{m-1}\right\|_{L^{2}}^{2} .
\end{aligned}
$$

(3) Error estimate for the velocity $\mathbf{u}$

Taking into account (3.2), from (5.12), (5.13), (5.14), (5.17) and (5.18), we have

$$
\begin{aligned}
\left(\delta_{t} \xi_{\mathbf{u}}^{m}, \overline{\mathbf{u}}\right)+\frac{D_{\mathbf{u}}}{\rho}\left(\nabla \xi_{\mathbf{u}}^{m}, \nabla \overline{\mathbf{u}}\right)= & \left(\omega_{\mathbf{u}}^{m}, \overline{\mathbf{u}}\right)-\left(\delta_{t} \theta_{\mathbf{u}}^{m}, \overline{\mathbf{u}}\right)-B\left(\mathbf{u}^{m}-\mathbf{u}^{m-1}, \mathbf{u}^{m}, \overline{\mathbf{u}}\right) \\
& -B\left(\xi_{\mathbf{u}}^{m-1}+\theta_{\mathbf{u}}^{m-1}, \mathbf{u}^{m}, \overline{\mathbf{u}}\right)-B\left(\mathbf{u}_{h}^{m-1}, \xi_{\mathbf{u}}^{m}+\theta_{\mathbf{u}}^{m}, \overline{\mathbf{u}}\right)+\frac{1}{\rho}\left(\xi_{\pi}^{m}, \nabla \cdot \overline{\mathbf{u}}\right) \\
& +\frac{1}{\rho}\left(\left(n^{m}-n^{m-1}\right) \nabla \phi, \overline{\mathbf{u}}\right)+\frac{1}{\rho}\left(\left(\xi_{n}^{m-1}+\theta_{n}^{m-1}\right) \nabla \phi, \overline{\mathbf{u}}\right), \\
\left(\bar{\pi}, \nabla \cdot \xi_{\mathbf{u}}^{m}\right)= & 0,
\end{aligned}
$$

for all $[\overline{\mathbf{u}}, \bar{\pi}] \in \mathcal{X}_{\mathbf{u}} \times \mathcal{X}_{\pi}$. Taking $\overline{\mathbf{u}}=\xi_{\mathbf{u}}^{m}$ in (5.39), $\bar{\pi}=\frac{1}{\rho} \xi_{\pi}^{m}$ in (5.40), using (3.13) and adding the resulting expressions, we obtain

$$
\begin{aligned}
\frac{1}{2} \delta_{t}\left\|\xi_{\mathbf{u}}^{m}\right\|_{L^{2}}^{2} & +\frac{\Delta t}{2}\left\|\delta_{t} \xi_{\mathbf{u}}^{m}\right\|_{L^{2}}^{2}+\frac{D_{\mathbf{u}}}{\rho}\left\|\nabla \xi_{\mathbf{u}}^{m}\right\|_{L^{2}}^{2}=\left(\omega_{\mathbf{u}}^{m}, \xi_{\mathbf{u}}^{m}\right)-\left(\delta_{t} \theta_{\mathbf{u}}^{m}, \xi_{\mathbf{u}}^{m}\right)-B\left(\mathbf{u}^{m}-\mathbf{u}^{m-1}, \mathbf{u}^{m}, \xi_{\mathbf{u}}^{m}\right) \\
& -B\left(\xi_{\mathbf{u}}^{m-1}+\theta_{\mathbf{u}}^{m-1}, \mathbf{u}^{m}, \xi_{\mathbf{u}}^{m}\right)-B\left(\mathbf{u}_{h}^{m-1}, \theta_{\mathbf{u}}^{m}, \xi_{\mathbf{u}}^{m}\right)+\frac{1}{\rho}\left(\left(n^{m}-n^{m-1}+\xi_{n}^{m-1}+\theta_{n}^{m-1}\right) \nabla \phi, \xi_{\mathbf{u}}^{m}\right) \\
& =\sum_{k=1}^{6} L_{k} .
\end{aligned}
$$

Then, we control the terms on the right hand side of (5.41) as follows: First, using the Hölder and Young inequalities, the Poincaré inequality (2.3) and (3.3), we get

$$
\begin{aligned}
L_{1} & \leq \frac{D_{\mathbf{u}}}{10 \rho}\left\|\nabla \xi_{\mathbf{u}}^{m}\right\|_{L^{2}}^{2}+\frac{C \rho}{D_{\mathbf{u}}}\left\|\omega_{\mathbf{u}}^{m}\right\|_{\left(H^{1}\right)^{\prime}}^{2} \leq \frac{D_{\mathbf{u}}}{10 \rho}\left\|\nabla \xi_{\mathbf{u}}^{m}\right\|_{L^{2}}^{2}+\frac{C \rho}{D_{\mathbf{u}}} \Delta t \int_{t_{m-1}}^{t_{m}}\left\|\mathbf{u}_{t t}(t)\right\|_{\left(H^{1}\right)^{\prime}}^{2} \mathrm{~d} t \\
L_{2} & \leq\left\|\xi_{\mathbf{u}}^{m}\right\|_{L^{2}}\left\|\left(\mathcal{I}-\mathbb{P}_{\mathbf{u}}\right) \delta_{t} \mathbf{u}^{m}\right\|_{L^{2}} \leq \frac{D_{\mathbf{u}}}{10 \rho}\left\|\nabla \xi_{\mathbf{u}}^{m}\right\|_{L^{2}}^{2}+\frac{C \rho}{D_{\mathbf{u}}} h^{2(r+1)}\left\|\left[\delta_{t} \mathbf{u}^{m}, \delta_{t} \pi^{m}\right]\right\|_{H^{r+1} \times H^{r}}^{2} \\
& \leq \frac{D_{\mathbf{u}}}{10 \rho}\left\|\nabla \xi_{\mathbf{u}}^{m}\right\|_{L^{2}}^{2}+\frac{C \rho}{D_{\mathbf{u}} \Delta t} h^{2(r+1)} \int_{t_{m-1}}^{t_{m}}\left\|\left[\mathbf{u}_{t}, \pi_{t}\right]\right\|_{H^{r+1} \times H^{r}}^{2} \mathrm{~d} t .
\end{aligned}
$$

From the definition of the skew-symmetric trilinear form (3.9), the Hölder, Young and Poincaré inequalities, (2.4) and (3.3), we have

$$
L_{3}+L_{4} \leq\left(\left\|\mathbf{u}^{m}-\mathbf{u}^{m-1}\right\|_{L^{2}}+\left\|\xi_{\mathbf{u}}^{m-1}\right\|_{L^{2}}+\left\|\theta_{\mathbf{u}}^{m-1}\right\|_{L^{2}}\right)\left\|\mathbf{u}^{m}\right\|_{L^{\infty} \cap W^{1,3}}\left\|\xi_{\mathbf{u}}^{m}\right\|_{H^{1}}
$$




$$
\begin{aligned}
\leq & \frac{D_{\mathbf{u}}}{10 \rho}\left\|\nabla \xi_{\mathbf{u}}^{m}\right\|_{L^{2}}^{2}+\frac{C \rho}{D_{\mathbf{u}}}\left(\left\|\mathbf{u}^{m}-\mathbf{u}^{m-1}\right\|_{L^{2}}^{2}+h^{2(r+1)}\left\|\left[\mathbf{u}^{m-1}, \pi^{m-1}\right]\right\|_{H^{r+1} \times H^{r}}^{2}\right. \\
& \left.+\left\|\xi_{\mathbf{u}}^{m-1}\right\|_{L^{2}}^{2}\right)\left\|\mathbf{u}^{m}\right\|_{H^{2}}^{2} .
\end{aligned}
$$

Also, using the definition of the skew-symmetric trilinear form (3.9), the Hölder, Young and Poincaré inequalities, (2.4), (3.3), (3.4) and (3.6), we get

$$
\begin{aligned}
L_{5}= & B\left(\xi_{\mathbf{u}}^{m-1}, \theta_{\mathbf{u}}^{m}, \xi_{\mathbf{u}}^{m}\right)-B\left(\mathbb{P}_{\mathbf{u}} \mathbf{u}^{m-1}, \theta_{\mathbf{u}}^{m}, \xi_{\mathbf{u}}^{m}\right) \\
\leq & \left\|\xi_{\mathbf{u}}^{m-1}\right\|_{L^{2}}\left\|\theta_{\mathbf{u}}^{m}\right\|_{L^{\infty} \cap W^{1,3}}\left\|\xi_{\mathbf{u}}^{m}\right\|_{H^{1}}+\left\|\mathbb{P}_{\mathbf{u}} \mathbf{u}^{m-1}\right\|_{L^{\infty} \cap W^{1,3}}\left\|\theta_{\mathbf{u}}^{m}\right\|_{L^{2}}\left\|\xi_{\mathbf{u}}^{m}\right\|_{H^{1}} \\
\leq & \frac{D_{\mathbf{u}}}{10 \rho}\left\|\nabla \xi_{\mathbf{u}}^{m}\right\|_{L^{2}}^{2}+\frac{C \rho}{D_{\mathbf{u}}}\left\|\left[\mathbf{u}^{m}, \pi^{m}\right]\right\|_{H^{2} \times H^{1}}^{2}\left\|\xi_{\mathbf{u}}^{m-1}\right\|_{L^{2}}^{2} \\
& +\frac{C \rho}{D_{\mathbf{u}}} h^{2(r+1)}\left\|\left[\mathbf{u}^{m-1}, \pi^{m-1}\right]\right\|_{H^{2} \times H^{1}}^{2}\left\|\left[\mathbf{u}^{m}, \pi^{m}\right]\right\|_{H^{r+1} \times H^{r}}^{2}, \\
L_{6} \leq & \frac{1}{\rho}\left\|\left[n^{m}-n^{m-1}, \xi_{n}^{m-1}, \theta_{n}^{m-1}\right]\right\|_{L^{2}}\|\nabla \phi\|_{L^{3}}\left\|\xi_{\mathbf{u}}^{m}\right\|_{H^{1}} \\
\leq & \frac{D_{\mathbf{u}}}{10 \rho}\left\|\nabla \xi_{\mathbf{u}}^{m}\right\|_{L^{2}}^{2}+\frac{C}{\rho D_{\mathbf{u}}}\left(\left\|n^{m}-n^{m-1}\right\|_{L^{2}}^{2}+h^{2\left(r_{1}+1\right)}\left\|n^{m-1}\right\|_{H^{r_{1}+1}}^{2}+\left\|\xi_{n}^{m-1}\right\|_{L^{2}}^{2}\right)\|\nabla \phi\|_{L^{3}}^{2} .
\end{aligned}
$$

Therefore, from (5.41) to (5.46), we arrive at

$$
\begin{aligned}
\frac{1}{2} \delta_{t}\left\|\xi_{\mathbf{u}}^{m}\right\|_{L^{2}}^{2} & +\frac{\Delta t}{2}\left\|\delta_{t} \xi_{\mathbf{u}}^{m}\right\|_{L^{2}}^{2}+\frac{D_{\mathbf{u}}}{2 \rho}\left\|\nabla \xi_{\mathbf{u}}^{m}\right\|_{L^{2}}^{2} \leq \frac{C \rho}{D_{\mathbf{u}}} \int_{t_{m-1}}^{t_{m}}\left(\Delta t\left\|\mathbf{u}_{t t}(t)\right\|_{\left(H^{1}\right)^{\prime}}^{2}+\frac{h^{2(r+1)}}{\Delta t}\left\|\left[\mathbf{u}_{t}, \pi_{t}\right]\right\|_{H^{r+1} \times H^{r}}^{2}\right) \mathrm{d} t \\
& +\frac{C \rho}{D_{\mathbf{u}}}\left(\left\|\mathbf{u}^{m}-\mathbf{u}^{m-1}\right\|_{L^{2}}^{2}+h^{2(r+1)}\left\|\left[\mathbf{u}^{m-1}, \pi^{m-1}\right]\right\|_{H^{r+1} \times H^{r}}^{2}+\left\|\xi_{\mathbf{u}}^{m-1}\right\|_{L^{2}}^{2}\right)\left\|\mathbf{u}^{m}\right\|_{H^{2}}^{2} \\
& +\frac{C \rho}{D_{\mathbf{u}}}\left(\left\|\left[\mathbf{u}^{m}, \pi^{m}\right]\right\|_{H^{2} \times H^{1}}^{2}\left\|\xi_{\mathbf{u}}^{m-1}\right\|_{L^{2}}^{2}+h^{2(r+1)}\left\|\left[\mathbf{u}^{m-1}, \pi^{m-1}\right]\right\|_{H^{2} \times H^{1}}^{2}\left\|\left[\mathbf{u}^{m}, \pi^{m}\right]\right\|_{H^{r+1} \times H^{r}}^{2}\right) \\
& +\frac{C}{\rho D_{\mathbf{u}}}\left(\left\|n^{m}-n^{m-1}\right\|_{L^{2}}^{2}+h^{2\left(r_{1}+1\right)}\left\|n^{m-1}\right\|_{H^{r_{1}+1}}^{2}+\left\|\xi_{n}^{m-1}\right\|_{L^{2}}^{2}\right)\|\nabla \phi\|_{L^{3}}^{2} .
\end{aligned}
$$

(4) Error estimate for the flux $\boldsymbol{\sigma}$

Taking into account $(3.5)_{3}$, from (5.10) and (5.14)-(5.17), we have

$$
\begin{aligned}
\left(\delta_{t} \xi_{\boldsymbol{\sigma}}^{m}, \overline{\boldsymbol{\sigma}}\right) & +D_{c}\left(\nabla \cdot \xi_{\boldsymbol{\sigma}}^{m}, \nabla \cdot \overline{\boldsymbol{\sigma}}\right)+D_{c}\left(\operatorname{rot} \xi_{\boldsymbol{\sigma}}^{m}, \operatorname{rot} \overline{\boldsymbol{\sigma}}\right)=\left(\omega_{\boldsymbol{\sigma}}^{m}, \overline{\boldsymbol{\sigma}}\right)-\left(\delta_{t} \theta_{\boldsymbol{\sigma}}^{m}, \overline{\boldsymbol{\sigma}}\right) \\
& +\left(\left(\mathbf{u}^{m}-\mathbf{u}^{m-1}\right) \cdot \boldsymbol{\sigma}^{m}+\mathbf{u}^{m-1}\left(\boldsymbol{\sigma}^{m}-\boldsymbol{\sigma}^{m-1}\right)+\left(\xi_{\mathbf{u}}^{m-1}+\theta_{\mathbf{u}}^{m-1}\right) \boldsymbol{\sigma}^{m-1}, \nabla \cdot \overline{\boldsymbol{\sigma}}\right) \\
& +\left(\mathbf{u}_{h}^{m-1}\left(\xi_{\boldsymbol{\sigma}}^{m-1}+\theta_{\boldsymbol{\sigma}}^{m-1}\right)+\gamma\left(n^{m}-n^{m-1}\right) c^{m}+\gamma\left(n^{m-1}+\alpha_{0}\right)\left(c^{m}-c^{m-1}\right), \nabla \cdot \overline{\boldsymbol{\sigma}}\right) \\
& +\gamma\left(\left(\xi_{n}^{m-1}+\theta_{n}^{m-1}\right) c_{h}^{m-1}+\left(n^{m-1}+\alpha_{0}\right)\left(\xi_{c}^{m-1}+\theta_{c}^{m-1}\right), \nabla \cdot \overline{\boldsymbol{\sigma}}\right)+D_{c}\left(\theta_{\boldsymbol{\sigma}}^{m}, \overline{\boldsymbol{\sigma}}\right),
\end{aligned}
$$

for all $\overline{\boldsymbol{\sigma}} \in \mathcal{X}_{\boldsymbol{\sigma}}$. Taking $\overline{\boldsymbol{\sigma}}=\xi_{\boldsymbol{\sigma}}^{m}$ in (5.48), we arrive at

$$
\begin{aligned}
\frac{1}{2} \delta_{t}\left\|\xi_{\boldsymbol{\sigma}}^{m}\right\|_{L^{2}}^{2} & +\frac{\Delta t}{2}\left\|\delta_{t} \xi_{\boldsymbol{\sigma}}^{m}\right\|_{L^{2}}^{2}+D_{c}\left\|\nabla \cdot \xi_{\boldsymbol{\sigma}}^{m}\right\|_{L^{2}}^{2}+D_{c}\left\|\operatorname{rot} \xi_{\boldsymbol{\sigma}}^{m}\right\|_{L^{2}}^{2}=\left(\omega_{\boldsymbol{\sigma}}^{m}, \xi_{\boldsymbol{\sigma}}^{m}\right)-\left(\delta_{t} \theta_{\boldsymbol{\sigma}}^{m}, \xi_{\boldsymbol{\sigma}}^{m}\right) \\
& +\left(\left(\mathbf{u}^{m}-\mathbf{u}^{m-1}\right) \cdot \boldsymbol{\sigma}^{m}+\mathbf{u}^{m-1}\left(\boldsymbol{\sigma}^{m}-\boldsymbol{\sigma}^{m-1}\right)+\left(\xi_{\mathbf{u}}^{m-1}+\theta_{\mathbf{u}}^{m-1}\right) \boldsymbol{\sigma}^{m-1}, \nabla \cdot \xi_{\boldsymbol{\sigma}}^{m}\right) \\
& +\left(\mathbf{u}_{h}^{m-1}\left(\xi_{\boldsymbol{\sigma}}^{m-1}+\theta_{\boldsymbol{\sigma}}^{m-1}\right), \nabla \cdot \xi_{\boldsymbol{\sigma}}^{m}\right)+\gamma\left(\left(n^{m}-n^{m-1}\right) c^{m}+\left(n^{m-1}+\alpha_{0}\right)\left(c^{m}-c^{m-1}\right), \nabla \cdot \xi_{\boldsymbol{\sigma}}^{m}\right) \\
& +\gamma\left(\left(\xi_{n}^{m-1}+\theta_{n}^{m-1}\right) c_{h}^{m-1}, \nabla \cdot \xi_{\boldsymbol{\sigma}}^{m}\right)+\gamma\left(\left(n^{m-1}+\alpha_{0}\right)\left(\xi_{c}^{m-1}+\theta_{c}^{m-1}\right), \nabla \cdot \xi_{\boldsymbol{\sigma}}^{m}\right)+D_{c}\left(\theta_{\boldsymbol{\sigma}}^{m}, \xi_{\boldsymbol{\sigma}}^{m}\right) \\
& =\sum_{k=1}^{8} R_{k} .
\end{aligned}
$$


Then, using the Hölder and Young inequalities, the equivalent norm in $\mathbf{H}_{\sigma}^{1}(\Omega)$ given in (2.2), as well as (3.3), (3.4) and (3.6)-(3.8), we control the terms on the right hand side of (5.49) as follows

$$
\begin{aligned}
& R_{1} \leq\left(\left\|\xi_{\boldsymbol{\sigma}}^{m}\right\|_{L^{2}}+\left\|\nabla \cdot \xi_{\boldsymbol{\sigma}}^{m}\right\|_{L^{2}}+\left\|\operatorname{rot} \xi_{\boldsymbol{\sigma}}^{m}\right\|_{L^{2}}\right)\left\|\omega_{\boldsymbol{\sigma}}^{m}\right\|_{\left(H^{1}\right)^{\prime}} \\
& \leq\left(\Delta t\left\|\delta_{t} \xi_{\boldsymbol{\sigma}}^{m}\right\|_{L^{2}}+\left\|\xi_{\boldsymbol{\sigma}}^{m-1}\right\|_{L^{2}}+\left\|\nabla \cdot \xi_{\boldsymbol{\sigma}}^{m}\right\|_{L^{2}}+\left\|\operatorname{rot} \xi_{\boldsymbol{\sigma}}^{m}\right\|_{L^{2}}\right)\left\|\omega_{\boldsymbol{\sigma}}^{m}\right\|_{\left(H^{1}\right)^{\prime}} \\
& \leq \frac{D_{c}}{6}\left\|\nabla \cdot \xi_{\boldsymbol{\sigma}}^{m}\right\|_{L^{2}}^{2}+\frac{D_{c}}{2}\left\|\operatorname{rot} \xi_{\boldsymbol{\sigma}}^{m}\right\|_{L^{2}}^{2}+\frac{\Delta t}{8}\left\|\delta_{t} \xi_{\boldsymbol{\sigma}}^{m}\right\|_{L^{2}}^{2}+\frac{1}{2}\left\|\xi_{\boldsymbol{\sigma}}^{m-1}\right\|_{L^{2}}^{2}+\left(\frac{C}{D_{c}}+C \Delta t+\frac{1}{2}\right)\left\|\omega_{\boldsymbol{\sigma}}^{m}\right\|_{\left(H^{1}\right)^{\prime}}^{2} \\
& \leq \frac{D_{c}}{6}\left\|\nabla \cdot \xi_{\boldsymbol{\sigma}}^{m}\right\|_{L^{2}}^{2}+\frac{D_{c}}{2}\left\|\operatorname{rot} \xi_{\boldsymbol{\sigma}}^{m}\right\|_{L^{2}}^{2}+\frac{\Delta t}{8}\left\|\delta_{t} \xi_{\boldsymbol{\sigma}}^{m}\right\|_{L^{2}}^{2}+\frac{1}{2}\left\|\xi_{\boldsymbol{\sigma}}^{m-1}\right\|_{L^{2}}^{2}+\gamma_{1} C \Delta t \int_{t_{m-1}}^{t_{m}}\left\|\boldsymbol{\sigma}_{t t}(t)\right\|_{\left(H^{1}\right)^{\prime}}^{2} \mathrm{~d} t, \\
& R_{2}+R_{8} \leq\left\|\xi_{\boldsymbol{\sigma}}^{m}\right\|_{L^{2}}\left\|\left(\mathcal{I}-\mathbb{P}_{\boldsymbol{\sigma}}\right) \delta_{t} \boldsymbol{\sigma}^{m}\right\|_{L^{2}}+D_{c}\left\|\xi_{\boldsymbol{\sigma}}^{m}\right\|_{L^{2}}\left\|\theta_{\boldsymbol{\sigma}}^{m}\right\|_{L^{2}} \\
& \leq\left(\left\|\left(\mathcal{I}-\mathbb{P}_{\boldsymbol{\sigma}}\right) \delta_{t} \boldsymbol{\sigma}^{m}\right\|_{L^{2}}+D_{c}\left\|\theta_{\boldsymbol{\sigma}}^{m}\right\|_{L^{2}}\right)\left(\Delta t\left\|\delta_{t} \xi_{\boldsymbol{\sigma}}^{m}\right\|_{L^{2}}+\left\|\xi_{\boldsymbol{\sigma}}^{m-1}\right\|_{L^{2}}\right) \\
& \leq \frac{\Delta t}{8}\left\|\delta_{t} \xi_{\boldsymbol{\sigma}}^{m}\right\|_{L^{2}}^{2}+\frac{1}{2}\left\|\xi_{\boldsymbol{\sigma}}^{m-1}\right\|_{L^{2}}^{2}+\gamma_{2} C h^{2\left(r_{3}+1\right)}\left[\left\|\delta_{t} \boldsymbol{\sigma}^{m}\right\|_{H^{r_{3}+1}}^{2}+D_{c}^{2}\left\|\boldsymbol{\sigma}^{m}\right\|_{H^{r_{3}+1}}^{2}\right] \\
& \leq \frac{\Delta t}{8}\left\|\delta_{t} \xi_{\boldsymbol{\sigma}}^{m}\right\|_{L^{2}}^{2}+\frac{1}{2}\left\|\xi_{\boldsymbol{\sigma}}^{m-1}\right\|_{L^{2}}^{2}+\gamma_{2} C h^{2\left(r_{3}+1\right)}\left[\frac{1}{\Delta t} \int_{t_{m-1}}^{t_{m}}\left\|\boldsymbol{\sigma}_{t}\right\|_{H^{r_{3}+1}}^{2} \mathrm{~d} t+D_{c}^{2}\left\|\boldsymbol{\sigma}^{m}\right\|_{H^{r_{3}+1}}^{2}\right], \\
& R_{3}+R_{5}+R_{7} \leq \frac{D_{c}}{6}\left\|\nabla \cdot \xi_{\boldsymbol{\sigma}}^{m}\right\|_{L^{2}}^{2}+\frac{C}{D_{c}}\left\|\left[\mathbf{u}^{m}-\mathbf{u}^{m-1}, \boldsymbol{\sigma}^{m}-\boldsymbol{\sigma}^{m-1}\right]\right\|_{L^{2}}^{2}\left\|\left[\boldsymbol{\sigma}^{m}, \mathbf{u}^{m-1}\right]\right\|_{L^{\infty}}^{2} \\
& +\frac{C}{D_{c}}\left(h^{2(r+1)}\left\|\left[\mathbf{u}^{m-1}, \pi^{m-1}\right]\right\|_{H^{r+1} \times H^{r}}^{2}+\left\|\xi_{\mathbf{u}}^{m-1}\right\|_{L^{2}}^{2}\right)\left\|\boldsymbol{\sigma}^{m-1}\right\|_{L^{\infty}}^{2} \\
& +\frac{\gamma^{2} C}{D_{c}}\left\|\left[n^{m}-n^{m-1}, c^{m}-c^{m-1}\right]\right\|_{L^{2}}^{2}\left\|\left[c^{m}, n^{m-1}+\alpha_{0}\right]\right\|_{L^{\infty}}^{2} \\
& +\frac{\gamma^{2} C}{D_{c}}\left(h^{2\left(r_{2}+1\right)}\left\|c^{m-1}\right\|_{H^{r_{2}+1}}^{2}+\left\|\xi_{c}^{m-1}\right\|_{L^{2}}^{2}\right)\left\|n^{m-1}+\alpha_{0}\right\|_{L^{\infty}}^{2}, \\
& R_{4}+R_{6}=\left(\left(\mathbb{P}_{\mathbf{u}} \mathbf{u}^{m-1}-\xi_{\mathbf{u}}^{m-1}\right)\left(\xi_{\boldsymbol{\sigma}}^{m-1}+\theta_{\boldsymbol{\sigma}}^{m-1}\right)+\gamma c_{h}^{m-1} \xi_{n}^{m-1}+\gamma\left(\mathbb{P}_{c} c^{m-1}-\xi_{c}^{m-1}\right) \theta_{n}^{m-1}, \nabla \cdot \xi_{\boldsymbol{\sigma}}^{m}\right) \\
& \leq \frac{D_{c}}{6}\left\|\nabla \cdot \xi_{\boldsymbol{\sigma}}^{m}\right\|_{L^{2}}^{2}+\frac{C}{D_{c}}\left\|\mathbb{P}_{\mathbf{u}} \mathbf{u}^{m-1}\right\|_{L^{\infty}}^{2}\left(\left\|\xi_{\boldsymbol{\sigma}}^{m-1}\right\|_{L^{2}}^{2}+\left\|\theta_{\boldsymbol{\sigma}}^{m-1}\right\|_{L^{2}}^{2}\right)+\frac{D_{\mathbf{u}}}{4 \rho}\left\|\nabla \xi_{\mathbf{u}}^{m-1}\right\|_{L^{2}}^{2} \\
& +\frac{C \rho}{D_{\mathbf{u}} D_{c}^{2}}\left\|\xi_{\mathbf{u}}^{m-1}\right\|_{L^{2}}^{2}\left(\left\|\xi_{\boldsymbol{\sigma}}^{m-1}\right\|_{L^{4}}^{4}+\left\|\theta_{\boldsymbol{\sigma}}^{m-1}\right\|_{L^{4}}^{4}\right)+\frac{D_{n}}{8}\left\|\xi_{n}^{m-1}\right\|_{H^{1}}^{2} \\
& +\frac{C \gamma^{4}}{D_{n} D_{c}^{2}}\left\|\xi_{n}^{m-1}\right\|_{L^{2}}^{2}\left\|c_{h}^{m-1}\right\|_{H^{1}}^{2}+\frac{C \gamma^{2}}{D_{c}}\left\|\mathbb{P}_{c} c^{m-1}\right\|_{L^{\infty}}^{2}\left\|\theta_{n}^{m-1}\right\|_{L^{2}}^{2} \\
& +\frac{C \gamma^{2}}{D_{c}}\left\|\theta_{n}^{m-1}\right\|_{L^{\infty}}^{2}\left\|\xi_{c}^{m-1}\right\|_{L^{2}}^{2} \\
& \leq \frac{D_{c}}{6}\left\|\nabla \cdot \xi_{\boldsymbol{\sigma}}^{m}\right\|_{L^{2}}^{2}+\frac{D_{\mathbf{u}}}{4 \rho}\left\|\nabla \xi_{\mathbf{u}}^{m-1}\right\|_{L^{2}}^{2}+\frac{D_{n}}{8}\left\|\xi_{n}^{m-1}\right\|_{H^{1}}^{2}+\frac{C \rho}{D_{\mathbf{u}} D_{c}^{2}}\left\|\xi_{\mathbf{u}}^{m-1}\right\|_{L^{2}}^{2}\left\|\left[\boldsymbol{\sigma}_{h}^{m-1}, \boldsymbol{\sigma}^{m-1}\right]\right\|_{H^{1}}^{2} \\
& +\frac{C}{D_{c}}\left(h^{2\left(r_{3}+1\right)}\left\|\boldsymbol{\sigma}^{m-1}\right\|_{H^{r_{3}+1}}^{2}+\left\|\xi_{\boldsymbol{\sigma}}^{m-1}\right\|_{L^{2}}^{2}\right)\left\|\mathbf{u}^{m-1}\right\|_{H^{2}}^{2}+\frac{C \gamma^{4}}{D_{n} D_{c}^{2}}\left\|\xi_{n}^{m-1}\right\|_{L^{2}}^{2}\left\|c_{h}^{m-1}\right\|_{H^{1}}^{2} \\
& +\frac{C \gamma^{2}}{D_{c}} h^{2\left(r_{1}+1\right)}\left\|c^{m-1}\right\|_{H^{2}}^{2}\left\|n^{m-1}\right\|_{H^{r_{1}+1}}^{2}+\frac{C \gamma^{2}}{D_{c}}\left\|n^{m-1}\right\|_{H^{2}}^{2}\left\|\xi_{c}^{m-1}\right\|_{L^{2}}^{2},
\end{aligned}
$$

where, in the first inequality of (5.53), the 2D interpolation inequality (2.5) was used. Therefore, from (5.49) to (5.53), by using the inductive hypothesis (5.1), we arrive at

$\frac{1}{2} \delta_{t}\left\|\xi_{\boldsymbol{\sigma}}^{m}\right\|_{L^{2}}^{2}+\frac{\Delta t}{4}\left\|\delta_{t} \xi_{\boldsymbol{\sigma}}^{m}\right\|_{L^{2}}^{2}+\frac{D_{c}}{2}\left\|\nabla \cdot \xi_{\boldsymbol{\sigma}}^{m}\right\|_{L^{2}}^{2}+\frac{D_{c}}{2}\left\|\operatorname{rot} \xi_{\boldsymbol{\sigma}}^{m}\right\|_{L^{2}}^{2} \leq \gamma_{1} C \Delta t \int_{t_{m-1}}^{t_{m}}\left\|\boldsymbol{\sigma}_{t t}(t)\right\|_{\left(H^{1}\right)^{\prime}}^{2} \mathrm{~d} t$ 


$$
\begin{aligned}
& +\left\|\xi_{\boldsymbol{\sigma}}^{m-1}\right\|_{L^{2}}^{2}+\gamma_{2} C h^{2\left(r_{3}+1\right)}\left[\frac{1}{\Delta t} \int_{t_{m-1}}^{t_{m}}\left\|\boldsymbol{\sigma}_{t}\right\|_{H^{r_{3}+1}}^{2} \mathrm{~d} t+D_{c}^{2}\left\|\boldsymbol{\sigma}^{m}\right\|_{H^{r_{3}+1}}^{2}\right] \\
& +\frac{C}{D_{c}}\left(\left\|\left[\mathbf{u}^{m}-\mathbf{u}^{m-1}, \boldsymbol{\sigma}^{m}-\boldsymbol{\sigma}^{m-1}\right]\right\|_{L^{2}}^{2}\left\|\left[\boldsymbol{\sigma}^{m}, \mathbf{u}^{m-1}\right]\right\|_{L^{\infty}}^{2}+h^{2(r+1)}\left\|\left[\mathbf{u}^{m-1}, \pi^{m-1}\right]\right\|_{H^{r+1} \times H^{r}}^{2}\left\|\boldsymbol{\sigma}^{m-1}\right\|_{L^{\infty}}^{2}\right) \\
& +\frac{C}{D_{c}}\left\|\xi_{\mathbf{u}}^{m-1}\right\|_{L^{2}}^{2}\left\|\boldsymbol{\sigma}^{m-1}\right\|_{L^{\infty}}^{2}+\frac{\gamma^{2} C}{D_{c}}\left\|\left[n^{m}-n^{m-1}, c^{m}-c^{m-1}\right]\right\|_{L^{2}}^{2}\left\|\left[c^{m}, n^{m-1}+\alpha_{0}\right]\right\|_{L^{\infty}}^{2} \\
& +\frac{\gamma^{2} C}{D_{c}}\left(h^{2\left(r_{2}+1\right)}\left\|c^{m-1}\right\|_{H^{r_{2}+1}}^{2}+\left\|\xi_{c}^{m-1}\right\|_{L^{2}}^{2}\right)\left\|n^{m-1}+\alpha_{0}\right\|_{L^{\infty}}^{2}+\frac{D_{\mathbf{u}}}{4 \rho}\left\|\nabla \xi_{\mathbf{u}}^{m-1}\right\|_{L^{2}}^{2}+\frac{D_{n}}{8}\left\|\xi_{n}^{m-1}\right\|_{H^{1}}^{2} \\
& +\frac{C \rho}{D_{\mathbf{u}} D_{c}^{2}}\left\|\xi_{\mathbf{u}}^{m-1}\right\|_{L^{2}}^{2}\left\|\left[\boldsymbol{\sigma}_{h}^{m-1}, \boldsymbol{\sigma}^{m-1}\right]\right\|_{H^{1}}^{2}+\frac{C}{D_{c}}\left(h^{2\left(r_{3}+1\right)}\left\|\boldsymbol{\sigma}^{m-1}\right\|_{H^{r_{3}+1}}^{2}+\left\|\xi_{\boldsymbol{\sigma}}^{m-1}\right\|_{L^{2}}^{2}\right)\left\|\mathbf{u}^{m-1}\right\|_{H^{2}}^{2} \\
& +\frac{C \gamma^{4}}{D_{n} D_{c}^{2}}\left\|\xi_{n}^{m-1}\right\|_{L^{2}}^{2}\left\|c_{h}^{m-1}\right\|_{H^{1}}^{2}+\frac{C \gamma^{2}}{D_{c}} h^{2\left(r_{1}+1\right)}\left\|c^{m-1}\right\|_{H^{2}}^{2}\left\|n^{m-1}\right\|_{H^{r_{1}+1}}^{2} \\
& +\frac{C \gamma^{2}}{D_{c}}\left\|n^{m-1}\right\|_{H^{2}}^{2}\left\|\xi_{c}^{m-1}\right\|_{L^{2}}^{2} .
\end{aligned}
$$

(5) Estimate for the terms $\left\|n^{m}-n^{m-1}\right\|_{L^{2}},\left\|c^{m}-c^{m-1}\right\|_{L^{2}},\left\|\mathbf{u}^{m}-\mathbf{u}^{m-1}\right\|_{L^{2}}$ and $\left.\| \boldsymbol{\sigma}^{m}-\boldsymbol{\sigma}^{m-1}\right] \|_{L^{2}}$

Observe that the following estimate holds

$$
\begin{aligned}
& \Delta t \sum_{m=1}^{r}\left\|\left[n^{m}-n^{m-1}, c^{m}-c^{m-1}, \mathbf{u}^{m}-\mathbf{u}^{m-1}, \boldsymbol{\sigma}^{m}-\boldsymbol{\sigma}^{m-1}\right]\right\|_{L^{2}}^{2} \\
& \quad \leq C(\Delta t)^{4}\left\|\left[n_{t t}, c_{t t}, \mathbf{u}_{t t}, \boldsymbol{\sigma}_{t t}\right]\right\|_{L^{2}\left(L^{2}\right)}^{2}+C(\Delta t)^{2}\left\|\left[n_{t}, c_{t}, \mathbf{u}_{t}, \boldsymbol{\sigma}_{t}\right]\right\|_{L^{2}\left(L^{2}\right)}^{2}
\end{aligned}
$$

Indeed,

$$
\left\|\omega_{n}^{m}\right\|_{L^{2}}=\left\|\delta_{t} n^{m}-\left(n_{t}\right)^{m}\right\|_{L^{2}}=\left\|\frac{1}{\Delta t}\left(n^{m}-n^{m-1}\right)-\left(n_{t}\right)^{m}\right\|_{L^{2}} \leq C(\Delta t)^{1 / 2}\left(\int_{t_{m-1}}^{t_{m}}\left\|n_{t t}(t)\right\|_{L^{2}}^{2} \mathrm{~d} t\right)^{1 / 2},
$$

where the last inequality was obtained as in (5.23), with the space norm in $L^{2}$ instead of $\left(H^{1}\right)^{\prime}$, the dual of $H^{1}$. Therefore, we deduce

$$
\Delta t \sum_{m=1}^{r}\left\|n^{m}-n^{m-1}\right\|_{L^{2}}^{2} \leq C(\Delta t)^{4}\left\|n_{t t}\right\|_{L^{2}\left(L^{2}\right)}^{2}+C(\Delta t)^{2}\left\|n_{t}\right\|_{L^{2}\left(L^{2}\right)}^{2} .
$$

Analogously, we obtain the estimate for $c, \mathbf{u}$ and $\boldsymbol{\sigma}$.

\subsubsection{Proof of Theorem 5.5}

Proof. The proof of (5.19) follows adding the four inequalities (5.29), (5.38), (5.47) and (5.54), multiplying the resulting expression by $\Delta t$, adding from $m=1$ to $m=s$, using (5.55), (2.8) and Lemmas 5.2, 5.3, and applying the discrete Gronwall Lemma 5.1 (recalling that $\left[\xi_{n}^{0}, \xi_{c}^{0}, \xi_{\mathbf{u}}^{0}, \xi_{\boldsymbol{\sigma}}^{0}\right]=[0,0, \mathbf{0}, \mathbf{0}]$ ).

Now we prove (5.20). Subtracting (5.40) at time $t=t_{m-1}$ from (5.40) at time $t=t_{m}$ and multiplying the resulting expression by $1 /(\rho \Delta t)$, we have that

$$
\frac{1}{\rho}\left(\bar{\pi}, \nabla \cdot \delta_{t} \xi_{\mathbf{u}}^{m}\right)=0 \quad \forall \bar{\pi} \in \mathcal{X}_{\pi} .
$$

Then, testing (5.39) by $\overline{\mathbf{u}}=\delta_{t} \xi_{\mathbf{u}}^{m},(5.56)$ by $\bar{\pi}=\xi_{\pi}^{m}$ and adding the resulting expressions, the terms $\frac{1}{\rho}\left(\xi_{\pi}^{m}, \nabla\right.$. $\left.\delta_{t} \xi_{\mathbf{u}}^{m}\right)$ cancel and we obtain

$$
\frac{D_{\mathbf{u}}}{2 \rho} \delta_{t}\left\|\xi_{\mathbf{u}}^{m}\right\|_{H^{1}}^{2}+\frac{\Delta t D_{\mathbf{u}}}{2 \rho}\left\|\delta_{t} \xi_{\mathbf{u}}^{m}\right\|_{H^{1}}^{2}+\left\|\delta_{t} \xi_{\mathbf{u}}^{m}\right\|_{L^{2}}^{2}=\left(\omega_{\mathbf{u}}^{m}-\delta_{t} \theta_{\mathbf{u}}^{m}, \delta_{t} \xi_{\mathbf{u}}^{m}\right)
$$




$$
\begin{aligned}
& -B\left(\mathbf{u}^{m}-\mathbf{u}^{m-1}, \mathbf{u}^{m}, \delta_{t} \xi_{\mathbf{u}}^{m}\right)-B\left(\xi_{\mathbf{u}}^{m-1}+\theta_{\mathbf{u}}^{m-1}, \mathbf{u}^{m}, \delta_{t} \xi_{\mathbf{u}}^{m}\right)-B\left(\mathbf{u}_{h}^{m-1}, \xi_{\mathbf{u}}^{m}+\theta_{\mathbf{u}}^{m}, \delta_{t} \xi_{\mathbf{u}}^{m}\right) \\
& +\frac{1}{\rho}\left(\left(n^{m}-n^{m-1}+\xi_{n}^{m-1}+\theta_{n}^{m-1}\right) \nabla \phi, \delta_{t} \xi_{\mathbf{u}}^{m}\right):=\left(g, \delta_{t} \xi_{\mathbf{u}}^{m}\right) \leq \frac{1}{2}\left(\left\|\delta_{t} \xi_{\mathbf{u}}^{m}\right\|_{L^{2}}^{2}+\|g\|_{L^{2}}^{2}\right) .
\end{aligned}
$$

We will give some details of the boundedness of $\|g\|_{L^{2}}$ : First, using the Hölder inequality, as well as the property (3.11), for all $\varphi \in L^{2}$, one has

$$
\begin{aligned}
\mid\left(\omega_{\mathbf{u}}^{m}-\right. & \left.\delta_{t} \theta_{\mathbf{u}}^{m}, \varphi\right)-B\left(\mathbf{u}^{m}-\mathbf{u}^{m-1}, \mathbf{u}^{m}, \varphi\right)+\frac{1}{\rho}\left(\left(n^{m}-n^{m-1}+\xi_{n}^{m-1}+\theta_{n}^{m-1}\right) \nabla \phi, \varphi\right) \mid \\
\leq & C\left(\left\|\omega_{\mathbf{u}}^{m}\right\|_{L^{2}}+\left\|\delta_{t} \theta_{\mathbf{u}}^{m}\right\|_{L^{2}}+\left\|\mathbf{u}^{m}-\mathbf{u}^{m-1}\right\|_{L^{2}}\left\|\nabla \mathbf{u}^{m}\right\|_{L^{\infty}}\right)\|\varphi\|_{L^{2}} \\
& +C\|\nabla \phi\|_{L^{\infty}}\left(\left\|n^{m}-n^{m-1}\right\|_{L^{2}}+\left\|\xi_{n}^{m-1}\right\|_{L^{2}}+\left\|\theta_{n}^{m-1}\right\|_{L^{2}}\right)\|\varphi\|_{L^{2}} .
\end{aligned}
$$

Now, using again the Hölder inequality, and integrating by parts conveniently, we have

$$
\left|B\left(\xi_{\mathbf{u}}^{m-1}+\theta_{\mathbf{u}}^{m-1}, \mathbf{u}^{m}, \varphi\right)\right| \leq C\left(\left\|\xi_{\mathbf{u}}^{m-1}\right\|_{H^{1}}+\left\|\theta_{\mathbf{u}}^{m-1}\right\|_{H^{1}}\right)\left\|\mathbf{u}^{m}\right\|_{L^{\infty} \cap W^{1,3}}\|\varphi\|_{L^{2}}
$$

and

$$
\begin{aligned}
& \left|B\left(\mathbf{u}_{h}^{m-1}, \xi_{\mathbf{u}}^{m}+\theta_{\mathbf{u}}^{m}, \varphi\right)\right|=\left|B\left(\mathbb{P}_{\mathbf{u}} \mathbf{u}^{m-1}, \xi_{\mathbf{u}}^{m}+\theta_{\mathbf{u}}^{m}, \varphi\right)-B\left(\xi_{\mathbf{u}}^{m-1}, \xi_{\mathbf{u}}^{m}+\theta_{\mathbf{u}}^{m}, \varphi\right)\right| \\
& \leq C\left\|\mathbb{P}_{\mathbf{u}} \mathbf{u}^{m-1}\right\|_{L^{\infty} \cap W^{1,3}}\left(\left\|\theta_{\mathbf{u}}^{m}\right\|_{H^{1}}+\left\|\xi_{\mathbf{u}}^{m}\right\|_{H^{1}}\right)\|\varphi\|_{L^{2}}+C\left\|\xi_{\mathbf{u}}^{m-1}\right\|_{H^{1}}\left\|\theta_{\mathbf{u}}^{m}\right\|_{L^{\infty} \cap W^{1,3}}\|\varphi\|_{L^{2}} \\
& +C\left\|\nabla \xi_{\mathbf{u}}^{m}\right\|_{L^{4}}\left\|\xi_{\mathbf{u}}^{m-1}\right\|_{L^{4}}\|\varphi\|_{L^{2}}+C\left\|\nabla \xi_{\mathbf{u}}^{m-1}\right\|_{L^{4}}\left\|\xi_{\mathbf{u}}^{m}\right\|_{L^{4}}\|\varphi\|_{L^{2}} \\
& \leq C\left\|\mathbb{P}_{\mathbf{u}} \mathbf{u}^{m-1}\right\|_{L^{\infty} \cap W^{1,3}}\left(\left\|\theta_{\mathbf{u}}^{m}\right\|_{H^{1}}+\left\|\xi_{\mathbf{u}}^{m}\right\|_{H^{1}}\right)\|\varphi\|_{L^{2}}+C\left\|\xi_{\mathbf{u}}^{m-1}\right\|_{H^{1}}\left\|\theta_{\mathbf{u}}^{m}\right\|_{L^{\infty} \cap W^{1,3}}\|\varphi\|_{L^{2}} \\
& +C\left\|\xi_{\mathbf{u}}^{m}\right\|_{W^{1,6}}^{1 / 2}\left\|\xi_{\mathbf{u}}^{m}\right\|_{H^{1}}^{1 / 2}\left\|\xi_{\mathbf{u}}^{m-1}\right\|_{H^{1}}\|\varphi\|_{L^{2}}+C\left\|\xi_{\mathbf{u}}^{m-1}\right\|_{W^{1,6}}^{1 / 2}\left\|\xi_{\mathbf{u}}^{m-1}\right\|_{H^{1}}^{1 / 2}\left\|\xi_{\mathbf{u}}^{m}\right\|_{H^{1}}\|\varphi\|_{L^{2}},
\end{aligned}
$$

where, in (5.60), the 2D interpolation inequality was used. Therefore from (5.58) to (5.60) we have

$$
\begin{aligned}
& \|g\|_{L^{2}}=\sup \left\{|(g, \varphi)|: \varphi \in L^{2}(\Omega),\|\varphi\|_{L^{2}} \leq 1\right\} \\
& \leq C\left(\left\|\omega_{\mathbf{u}}^{m}\right\|_{L^{2}}+\left\|\delta_{t} \theta_{\mathbf{u}}^{m}\right\|_{L^{2}}+\left\|\mathbf{u}^{m}-\mathbf{u}^{m-1}\right\|_{L^{2}}\left\|\nabla \mathbf{u}^{m}\right\|_{L^{\infty}}\right. \\
& +\left(\left\|\xi_{\mathbf{u}}^{m-1}\right\|_{H^{1}}+\left\|\theta_{\mathbf{u}}^{m-1}\right\|_{H^{1}}\right)\left\|\mathbf{u}^{m}\right\|_{L^{\infty} \cap W^{1,3}}+\left\|\xi_{\mathbf{u}}^{m-1}\right\|_{H^{1}}\left\|\theta_{\mathbf{u}}^{m}\right\|_{L^{\infty} \cap W^{1,3}} \\
& +\left\|\mathbb{P}_{\mathbf{u}} \mathbf{u}^{m-1}\right\|_{L^{\infty} \cap W^{1,3}}\left(\left\|\theta_{\mathbf{u}}^{m}\right\|_{H^{1}}+\left\|\xi_{\mathbf{u}}^{m}\right\|_{H^{1}}\right)+\left\|\xi_{\mathbf{u}}^{m}\right\|_{W^{1,6}}^{1 / 2}\left\|\xi_{\mathbf{u}}^{m}\right\|_{H^{1}}^{1 / 2}\left\|\xi_{\mathbf{u}}^{m-1}\right\|_{H^{1}} \\
& \left.+\left\|\xi_{\mathbf{u}}^{m-1}\right\|_{W^{1,6}}^{1 / 2}\left\|\xi_{\mathbf{u}}^{m-1}\right\|_{H^{1}}^{1 / 2}\left\|\xi_{\mathbf{u}}^{m}\right\|_{H^{1}}+\left(\left\|n^{m}-n^{m-1}\right\|_{L^{2}}+\left\|\xi_{n}^{m-1}\right\|_{L^{2}}+\left\|\theta_{n}^{m-1}\right\|_{L^{2}}\right)\|\nabla \phi\|_{L^{\infty}}\right) .
\end{aligned}
$$

Therefore from (5.57) to (5.61), we arrive at

$$
\begin{aligned}
& \frac{D_{\mathbf{u}}}{2 \rho} \delta_{t}\left\|\xi_{\mathbf{u}}^{m}\right\|_{H^{1}}^{2}+\frac{1}{2}\left\|\delta_{t} \xi_{\mathbf{u}}^{m}\right\|_{L^{2}}^{2} \leq \frac{1}{2}\|g\|_{L^{2}}^{2} \leq C\left(\left\|\omega_{\mathbf{u}}^{m}\right\|_{L^{2}}^{2}+\left\|\delta_{t} \theta_{\mathbf{u}}^{m}\right\|_{L^{2}}^{2}+\left\|\mathbf{u}^{m}-\mathbf{u}^{m-1}\right\|_{L^{2}}^{2}\left\|\nabla \mathbf{u}^{m}\right\|_{L^{\infty}}^{2}\right. \\
& +\left(\left\|\xi_{\mathbf{u}}^{m-1}\right\|_{H^{1}}^{2}+\left\|\theta_{\mathbf{u}}^{m-1}\right\|_{H^{1}}^{2}\right)\left\|\mathbf{u}^{m}\right\|_{L^{\infty} \cap W^{1,3}}^{2}+\left\|\xi_{\mathbf{u}}^{m-1}\right\|_{H^{1}}^{2}\left\|\theta_{\mathbf{u}}^{m}\right\|_{L^{\infty} \cap W^{1,3}}^{2} \\
& +\left\|\mathbb{P}_{\mathbf{u}} \mathbf{u}^{m-1}\right\|_{L^{\infty} \cap W^{1,3}}^{2}\left(\left\|\theta_{\mathbf{u}}^{m}\right\|_{H^{1}}^{2}+\left\|\xi_{\mathbf{u}}^{m}\right\|_{H^{1}}^{2}\right)+\varepsilon\left(\left\|\xi_{\mathbf{u}}^{m}\right\|_{W^{1,6}}^{2}+\left\|\xi_{\mathbf{u}}^{m-1}\right\|_{W^{1,6}}^{2}\right) \\
& +C_{\varepsilon}\left\|\xi_{\mathbf{u}}^{m}\right\|_{H^{1}}^{2}\left(\left\|\xi_{\mathbf{u}}^{m-1}\right\|_{H^{1}}^{4}+\left\|\xi_{\mathbf{u}}^{m-1}\right\|_{H^{1}}^{2}\left\|\xi_{\mathbf{u}}^{m}\right\|_{H^{1}}^{2}\right) \\
& \left.+\left(\left\|n^{m}-n^{m-1}\right\|_{L^{2}}^{2}+\left\|\xi_{n}^{m-1}\right\|_{L^{2}}^{2}+\left\|\theta_{n}^{m-1}\right\|_{L^{2}}^{2}\right)\|\nabla \phi\|_{L^{\infty}}^{2}\right) \text {. }
\end{aligned}
$$

On the other hand, applying Lemma 11 of [12] to (5.39) and (5.40), we have

$$
\left\|\left[\xi_{\mathbf{u}}^{m}, \xi_{\pi}^{m}\right]\right\|_{W^{1,6} \times L^{6}}^{2} \leq C\left(\left\|\delta_{t} \xi_{\mathbf{u}}^{m}\right\|_{L^{2}}^{2}+\|g\|_{L^{2}}^{2}\right)
$$

with $g$ as in (5.57) and (5.62). Thus, adding (5.62) with $\lambda(5.63)$ (for $0<\lambda<1$ ), we arrive at

$$
\frac{D_{\mathbf{u}}}{2 \rho} \delta_{t}\left\|\xi_{\mathbf{u}}^{m}\right\|_{H^{1}}^{2}+\frac{1}{2}\left\|\delta_{t} \xi_{\mathbf{u}}^{m}\right\|_{L^{2}}^{2}+\lambda\left\|\left[\xi_{\mathbf{u}}^{m}, \xi_{\pi}^{m}\right]\right\|_{W^{1,6} \times L^{6}}^{2} \leq C\left(\lambda\left\|\delta_{t} \xi_{\mathbf{u}}^{m}\right\|_{L^{2}}^{2}+\left\|\omega_{\mathbf{u}}^{m}\right\|_{L^{2}}^{2}+\left\|\delta_{t} \theta_{\mathbf{u}}^{m}\right\|_{L^{2}}^{2}\right.
$$




$$
\begin{aligned}
& +\left\|\mathbf{u}^{m}-\mathbf{u}^{m-1}\right\|_{L^{2}}^{2}\left\|\nabla \mathbf{u}^{m}\right\|_{L^{\infty}}^{2}+\left(\left\|\xi_{\mathbf{u}}^{m-1}\right\|_{H^{1}}^{2}+\left\|\theta_{\mathbf{u}}^{m-1}\right\|_{H^{1}}^{2}\right)\left\|\mathbf{u}^{m}\right\|_{L^{\infty} \cap W^{1,3}}^{2} \\
& +\left\|\xi_{\mathbf{u}}^{m-1}\right\|_{H^{1}}^{2}\left\|\theta_{\mathbf{u}}^{m}\right\|_{L^{\infty} \cap W^{1,3}}^{2} \\
& +\left\|\mathbb{P}_{\mathbf{u}} \mathbf{u}^{m-1}\right\|_{L^{\infty}}^{2} \cap W^{1,3}\left(\left\|\theta_{\mathbf{u}}^{m}\right\|_{H^{1}}^{2}+\left\|\xi_{\mathbf{u}}^{m}\right\|_{H^{1}}^{2}\right)+\varepsilon\left(\left\|\xi_{\mathbf{u}}^{m}\right\|_{W^{1,6}}^{2}+\left\|\xi_{\mathbf{u}}^{m-1}\right\|_{W^{1,6}}^{2}\right) \\
& +C_{\varepsilon}\left\|\xi_{\mathbf{u}}^{m}\right\|_{H^{1}}^{2}\left(\left\|\xi_{\mathbf{u}}^{m-1}\right\|_{H^{1}}^{4}+\left\|\xi_{\mathbf{u}}^{m-1}\right\|_{H^{1}}^{2}\left\|\xi_{\mathbf{u}}^{m}\right\|_{H^{1}}^{2}\right) \\
& \left.+\left(\left\|n^{m}-n^{m-1}\right\|_{L^{2}}^{2}+\left\|\xi_{n}^{m-1}\right\|_{L^{2}}^{2}+\left\|\theta_{n}^{m-1}\right\|_{L^{2}}^{2}\right)\|\nabla \phi\|_{L^{\infty}}^{2}\right) .
\end{aligned}
$$

We take $\lambda$ small enough in order to absorb the term $C \lambda\left\|\delta_{t} \xi_{\mathbf{u}}^{m}\right\|_{L^{2}}^{2}$ at the right hand side, and $\varepsilon$ small enough with respect to $\lambda$. Moreover, observe that

$$
\left\|\xi_{\mathbf{u}}^{m-1}\right\|_{H^{1}}^{4}+\left\|\xi_{\mathbf{u}}^{m-1}\right\|_{H^{1}}^{2}\left\|\xi_{\mathbf{u}}^{m}\right\|_{H^{1}}^{2} \leq C .
$$

Indeed, recalling that $h_{\max }:=\max \left\{h^{r_{1}+1}, h^{r_{2}+1}, h^{r_{3}+1}, h^{r+1}\right\}$, from estimate (5.19) we have in particular that $\left\|\xi_{\mathbf{u}}^{m}\right\|_{H^{1}}^{2} \leq C(T)\left(\Delta t+\frac{1}{\Delta t} h_{\max }^{2}\right)$, which implies that

$$
\left\|\xi_{\mathbf{u}}^{m-1}\right\|_{H^{1}}^{4}+\left\|\xi_{\mathbf{u}}^{m-1}\right\|_{H^{1}}^{2}\left\|\xi_{\mathbf{u}}^{m}\right\|_{H^{1}}^{2} \leq C(T)\left(\Delta t+\frac{1}{\Delta t} h_{\max }^{2}\right)^{2} .
$$

Therefore, we conclude (5.65) under the hypothesis

$$
\frac{h_{\max }^{4}}{(\Delta t)^{2}} \leq C
$$

On the other hand, from (5.19) we also have $\left\|\xi_{\mathbf{u}}^{m}\right\|_{L^{2}}^{2} \leq C(T)\left((\Delta t)^{2}+h_{\max }^{2}\right)$ for each $m$. Therefore, by using the inverse inequality $\left\|\xi_{\mathbf{u}}^{m}\right\|_{H^{1}} \leq h^{-1}\left\|\xi_{\mathbf{u}}^{m}\right\|_{L^{2}}$ we obtain

$$
\left\|\xi_{\mathbf{u}}^{m-1}\right\|_{H^{1}}^{4}+\left\|\xi_{\mathbf{u}}^{m-1}\right\|_{H^{1}}^{2}\left\|\xi_{\mathbf{u}}^{m}\right\|_{H^{1}}^{2} \leq C(T) \frac{1}{h^{4}}\left((\Delta t)^{2}+h_{\max }^{2}\right)^{2} .
$$

Therefore, we conclude (5.65) under the hypothesis

$$
\frac{(\Delta t)^{4}}{h^{4}} \leq C
$$

Thus, if $\Delta t$ and $h$ are less than or equal to 1 , we conclude (5.65) because for any choice of $(\Delta t, h)$ either $(5.66)$ or (5.67) holds. Therefore, multiplyng (5.64) by $\Delta t$, adding from $m=1$ to $m=s$, bounding the terms $\left\|\omega_{\mathbf{u}}^{m}\right\|_{L^{2}}^{2}$ and $\left\|\delta_{t} \theta_{\mathbf{u}}^{m}\right\|_{L^{2}}^{2}$ as in (5.42) and (5.43) respectively, using (2.8), (3.3), (3.4), (3.6) $1,(5.55),(5.19)$ and taking into account that $\xi_{\mathbf{u}}^{0}=\mathbf{0}$, we conclude $(5.20)$.

Remark 5.9. Notice that estimate (5.60) is independent of the dimension; that is, in the three-dimensional case, the 3D interpolation inequality (2.6) can also be used and (5.60) remains true.

It is clear that the error estimates in Theorem 5.5 were derived under the inductive hypothesis (5.1). Now we have to check it. We derive (5.1) by using (5.19) recursively. First, observe that $\left\|\mathbb{P}_{\boldsymbol{\sigma}} \boldsymbol{\sigma}^{m-1}\right\|_{H^{1}} \leq\|\boldsymbol{\sigma}\|_{L^{\infty}\left(H^{1}\right)}:=C_{0}$ for all $m \geq 1$, from which we deduce that $\left\|\boldsymbol{\sigma}_{h}^{0}\right\|_{H^{1}}=\left\|\mathbb{P}_{\boldsymbol{\sigma}} \boldsymbol{\sigma}_{0}\right\|_{H^{1}} \leq C_{0} \leq C_{0}+1:=K$. Now, notice that

$$
\left\|\boldsymbol{\sigma}_{h}^{m-1}\right\|_{H^{1}} \leq\left\|\xi_{\boldsymbol{\sigma}}^{m-1}\right\|_{H^{1}}+\left\|\mathbb{P}_{\boldsymbol{\sigma}} \boldsymbol{\sigma}^{m-1}\right\|_{H^{1}}
$$

Then, it is enough to show that $\left\|\xi_{\sigma}^{m-1}\right\|_{H^{1}} \leq 1$, for each $m \geq 2$. For that, we consider two cases: First, if $\frac{(\Delta t)^{1 / 2}}{h} \leq$ $C$, then, by using the inverse inequality $\left\|\xi_{\boldsymbol{\sigma}}^{m}\right\|_{H^{1}} \leq h^{-1}\left\|\xi_{\boldsymbol{\sigma}}^{m}\right\|_{L^{2}}$, denoting $h_{\max }:=\max \left\{h^{r_{1}+1}, h^{r_{2}+1}, h^{r_{3}+1}, h^{r+1}\right\}$, recalling that $h_{\max }=h^{k}$, (for some $k \geq 2$ ), and from the calculation of the norm $l^{\infty}\left(L^{2}\right)$ in (5.19), we get

$$
\left\|\xi_{\boldsymbol{\sigma}}^{1}\right\|_{H^{1}} \leq \frac{1}{h}\left\|\xi_{\boldsymbol{\sigma}}^{1}\right\|_{L^{2}} \leq C\left(T,\left\|\boldsymbol{\sigma}_{h}^{0}\right\|_{H^{1}}\right) \frac{1}{h}\left(\Delta t+h_{\max }\right) \leq C\left(T,\left\|\boldsymbol{\sigma}_{h}^{0}\right\|_{H^{1}}\right)\left(C(\Delta t)^{1 / 2}+h^{k-1}\right) .
$$


On the other hand, if $\frac{(\Delta t)^{1 / 2}}{h}$ is not bounded, then $\frac{h_{\max }}{\Delta t} \leq \frac{h^{2}}{\Delta t} \leq C$; thus, from the calculation of the norm $l^{2}\left(H^{1}\right)$ in (5.19), we get

$$
\left\|\xi_{\boldsymbol{\sigma}}^{1}\right\|_{H^{1}}^{2} \leq C\left(T,\left\|\boldsymbol{\sigma}_{h}^{0}\right\|_{H^{1}}\right) \frac{1}{\Delta t}\left((\Delta t)^{2}+h_{\max }^{2}\right) \leq C\left(T,\left\|\boldsymbol{\sigma}_{h}^{0}\right\|_{H^{1}}\right)\left(\Delta t+C h_{\max }\right) .
$$

In any case, assuming $\Delta t$ and $h$ small enough (without any additional restriction relating the discrete parameters $(\Delta t, h)$ ), we conclude that $\left\|\xi_{\boldsymbol{\sigma}}^{1}\right\|_{H^{1}} \leq 1$, which implies $\left\|\boldsymbol{\sigma}_{h}^{1}\right\|_{H^{1}} \leq K$. Analogously, by using $\left\|\boldsymbol{\sigma}_{h}^{1}\right\|_{H^{1}} \leq K$, we can obtain $\left\|\xi_{\boldsymbol{\sigma}}^{2}\right\|_{H^{1}} \leq 1$, and therefore, $\left\|\boldsymbol{\sigma}_{h}^{2}\right\|_{H^{1}} \leq K$. Arguing recursively we conclude that $\left\|\boldsymbol{\sigma}_{h}^{m-1}\right\|_{H^{1}} \leq K$, for all $m \geq 1$.

Remark 5.10. For the scheme (3.15), the inductive hypothesis (5.1) used to prove the convergence was verified only by assuming $\Delta t$ and $h$ small enough (without any additional restriction relating the discrete parameters $(\Delta t, h))$. On the other hand, if we do not use the auxiliary variable $\boldsymbol{\sigma}=\nabla c$, the inductive hypothesis required to control the chemotaxis term would fall on $c$; in this case, it would be

$$
\left\|\nabla c_{h}^{n-1}\right\|_{L^{6}} \leq K, \quad \forall n \geq 1 .
$$

Therefore, given that in the error estimates for $c$ does not appear the $W^{1,6}$-norm, in order to prove recursively the inductive hypothesis (5.71), we only could use the error estimates for $c$ in the $l^{\infty}\left(L^{2}\right)$ and $l^{2}\left(H^{1}\right)$-norms and the inverse inequalities

$$
\begin{aligned}
\left\|c_{h}^{n}\right\|_{W^{1,6}} \leq C h^{-2 / 3}\left\|c_{h}^{n}\right\|_{H^{1}}, & \forall c_{h}^{n} \in \mathcal{X}_{c}, \\
\left\|c_{h}^{n}\right\|_{W^{1,6}} \leq C h^{-5 / 3}\left\|c_{h}^{n}\right\|_{L^{2}}, & \forall c_{h}^{n} \in \mathcal{X}_{c} .
\end{aligned}
$$

It is straighforward to check that if we use the $l^{2}\left(H^{1}\right)$-norm and the inverse inequality (5.72), the relations $\frac{(\Delta t)^{a}}{h^{4}} \leq C_{1}$ and $\frac{h^{b}}{(\Delta t)^{3}} \leq C_{2}$, for some real numbers $a, b, C_{1}, C_{2}>0$ with $a<3$ and $b<8$, must be satisfied. However, these conditions are not necessarily satisfied for any $\Delta t$ and $h$ small enough. On the other hand, if we use the $l^{\infty}\left(L^{2}\right)$-norm and the inverse inequality (5.73), in addition to assumption $h$ and $\Delta t$ small enough, the relation $\frac{(\Delta t)^{d}}{h^{5}} \leq C$, for some $d, C>0$ with $d<3$, must be satisfied. In both cases, the conditions on the discrete parameters to ensure convergence are more restrictive than those required to ensure convergence of the scheme using the variable $\boldsymbol{\sigma}=\nabla c$.

\section{Some COMments on the three-Dimensional CASE}

The results obtained in Section 5 can be proved also in the three-dimensional case. Observe that the unconditional well-posedness of the scheme (3.15) and the mass-conservation property (2.10) were proved independently on the dimension (see Lem. 4.1 and Thm. 4.2, respectively). Now, in order to analyze the convergence in the $3 \mathrm{D}$ case, we need to modify the inductive hypothesis (5.1) by the following one: there exists a positive constant $K>0$, independent of $m$, such that

$$
\left\|\left[\boldsymbol{\sigma}_{h}^{m-1}, c_{h}^{m-1}\right]\right\|_{H^{1}} \leq K, \quad \forall m \geq 1 .
$$

Here it is worthwhile to observe that the inductive hypothesis $\left\|c_{h}^{m-1}\right\|_{H^{1}} \leq K$, for all $m \geq 1$, comes from using the interpolation inequality (2.6) in place of (2.5) to bound the term $\gamma\left(c_{h}^{m-1} \xi_{n}^{m-1}, \nabla \cdot \xi_{\boldsymbol{\sigma}}^{m}\right)$ in (5.49) in the three-dimensional case. Then, we can prove error estimates for any solution of the scheme (3.15), with respect to a sufficiently regular solution of (2.13).

Theorem 6.1. Assume that there exists a sufficiently regular solution of (2.13). If the inductive hypothesis (6.1) is satisfied, then the following error estimates hold

$$
\left\|\left[\xi_{n}^{m}, \xi_{c}^{m}, \xi_{\mathbf{u}}^{m}, \xi_{\boldsymbol{\sigma}}^{m}\right]\right\|_{l^{\infty}\left(L^{2}\right) \cap l^{2}\left(H^{1}\right)} \leq C(T)\left(\Delta t+\max \left\{h^{r_{1}+1}, h^{r_{2}+1}, h^{r_{3}+1}, h^{r+1}\right\}\right),
$$




$$
\left\|\xi_{\mathbf{u}}^{m}\right\|_{l^{\infty}\left(H^{1}\right) \cap L^{2}\left(W^{1,6}\right)}+\left\|\xi_{\pi}^{m}\right\|_{l^{2}\left(L^{6}\right)} \leq C(T)\left(\Delta t+\max \left\{h^{r_{1}+1}, h^{r_{2}+1}, h^{r_{3}+1}, h^{r}\right\}\right),
$$

where the constant $C(T)>0$ is independent of $m, \Delta t$ and $h$.

Proof. The proof follows as in Theorem 5.5, but in this case, in order to bound the terms in the estimates (5.28) and (5.53), we need to use the 3D interpolation inequality (2.6) and the inductive hypothesis (6.1), as follows:

$$
\begin{aligned}
I_{9}= & \chi\left(\xi_{n}^{m-1} \boldsymbol{\sigma}_{h}^{m-1}, \nabla \xi_{n}^{m}\right)-\chi\left(\theta_{n}^{m-1} \xi_{\boldsymbol{\sigma}}^{m-1}, \nabla \xi_{n}^{m}\right)+\chi\left(\theta_{n}^{m-1} \mathbb{P}_{\boldsymbol{\sigma}} \boldsymbol{\sigma}^{m-1}, \nabla \xi_{n}^{m}\right) \\
\leq & \chi\left(\left\|\xi_{n}^{m-1}\right\|_{L^{2}}^{1 / 2}\left\|\xi_{n}^{m-1}\right\|_{H^{1}}^{1 / 2}\left\|\boldsymbol{\sigma}_{h}^{m-1}\right\|_{L^{6}}+\left\|\xi_{\boldsymbol{\sigma}}^{m-1}\right\|_{L^{2}}\left\|\theta_{n}^{m-1}\right\|_{L^{\infty}}+\left\|\mathbb{P}_{\boldsymbol{\sigma}} \boldsymbol{\sigma}^{m-1}\right\|_{L^{\infty}}\left\|\theta_{n}^{m-1}\right\|_{L^{2}}\right)\left\|\xi_{n}^{m}\right\|_{H^{1}} \\
\leq & \frac{D_{n}}{12}\left\|\xi_{n}^{m}\right\|_{H^{1}}^{2}+\frac{D_{n}}{4}\left\|\xi_{n}^{m-1}\right\|_{H^{1}}^{2}+\frac{C \chi^{4}}{D_{n}^{3}}\left\|\xi_{n}^{m-1}\right\|_{L^{2}}^{2} \\
& +\frac{C \chi^{2}}{D_{n}}\left\|n^{m-1}\right\|_{H^{2}}^{2}\left\|\xi_{\boldsymbol{\sigma}}^{m-1}\right\|_{L^{2}}^{2}+\frac{C \chi^{2}}{D_{n}} h^{2\left(r_{1}+1\right)}\left\|\boldsymbol{\sigma}^{m-1}\right\|_{H^{2}}^{2}\left\|n^{m-1}\right\|_{H^{r_{1}+1}}^{2},
\end{aligned}
$$

and

$$
\begin{aligned}
R_{4}+R_{6}= & \left(\left(\mathbb{P}_{\mathbf{u}} \mathbf{u}^{m-1}-\xi_{\mathbf{u}}^{m-1}\right)\left(\xi_{\boldsymbol{\sigma}}^{m-1}+\theta_{\boldsymbol{\sigma}}^{m-1}\right)+\gamma c_{h}^{m-1} \xi_{n}^{m-1}+\gamma\left(\mathbb{P}_{c} c^{m-1}-\xi_{c}^{m-1}\right) \theta_{n}^{m-1}, \nabla \cdot \xi_{\boldsymbol{\sigma}}^{m}\right) \\
\leq & \frac{D_{c}}{6}\left\|\nabla \cdot \xi_{\boldsymbol{\sigma}}^{m}\right\|_{L^{2}}^{2}+\frac{C}{D_{c}}\left\|\mathbb{P}_{\mathbf{u}} \mathbf{u}^{m-1}\right\|_{L^{\infty}}^{2}\left(\left\|\xi_{\boldsymbol{\sigma}}^{m-1}\right\|_{L^{2}}^{2}+\left\|\theta_{\boldsymbol{\sigma}}^{m-1}\right\|_{L^{2}}^{2}\right)+\frac{D_{\mathbf{u}}}{4 \rho}\left\|\nabla \xi_{\mathbf{u}}^{m-1}\right\|_{L^{2}}^{2} \\
& +\frac{C \rho}{D_{\mathbf{u}} D_{c}^{2}}\left\|\xi_{\mathbf{u}}^{m-1}\right\|_{L^{2}}^{2}\left(\left\|\xi_{\boldsymbol{\sigma}}^{m-1}\right\|_{L^{6}}^{4}+\left\|\theta_{\boldsymbol{\sigma}}^{m-1}\right\|_{L^{6}}^{4}\right)+\frac{D_{n}}{8}\left\|\xi_{n}^{m-1}\right\|_{H^{1}}^{2}+\frac{C \gamma^{4}}{D_{n} D_{c}^{2}}\left\|\xi_{n}^{m-1}\right\|_{L^{2}}^{2}\left\|c_{h}^{m-1}\right\|_{L^{6}}^{4} \\
& +\frac{C \gamma^{2}}{D_{c}}\left\|\mathbb{P}_{c} c^{m-1}\right\|_{L^{\infty}}^{2}\left\|\theta_{n}^{m-1}\right\|_{L^{2}}^{2}+\frac{C \gamma^{2}}{D_{c}}\left\|\theta_{n}^{m-1}\right\|_{L^{\infty}}^{2}\left\|\xi_{c}^{m-1}\right\|_{L^{2}}^{2} \\
\leq & \frac{D_{c}}{6}\left\|\nabla \cdot \xi_{\boldsymbol{\sigma}}^{m}\right\|_{L^{2}}^{2}+\frac{D_{\mathbf{u}}}{4 \rho}\left\|\nabla \xi_{\mathbf{u}}^{m-1}\right\|_{L^{2}}^{2}+\frac{D_{n}}{8}\left\|\xi_{n}^{m-1}\right\|_{H^{1}}^{2}+\frac{C \rho}{D_{\mathbf{u}} D_{c}^{2}}\left\|\xi_{\mathbf{u}}^{m-1}\right\|_{L^{2}}^{2}\left\|\left[\boldsymbol{\sigma}_{h}^{m-1}, \boldsymbol{\sigma}^{m-1}\right]\right\|_{H^{1}}^{2} \\
& +\frac{C}{D_{c}}\left(h^{2\left(r_{3}+1\right)}\left\|\boldsymbol{\sigma}^{m-1}\right\|_{H^{r_{3}+1}}^{2}+\left\|\xi_{\boldsymbol{\sigma}}^{m-1}\right\|_{L^{2}}^{2}\right)\left\|\mathbf{u}^{m-1}\right\|_{H^{2}}^{2}+\frac{C \gamma^{4}}{D_{n} D_{c}^{2}}\left\|\xi_{n}^{m-1}\right\|_{L^{2}}^{2}\left\|c_{h}^{m-1}\right\|_{H^{1}}^{2} \\
& +\frac{C \gamma^{2}}{D_{c}} h^{2\left(r_{1}+1\right)}\left\|c^{m-1}\right\|_{H^{2}}^{2}\left\|n^{m-1}\right\|_{H^{r_{1}+1}}^{2}+\frac{C \gamma^{2}}{D_{c}}\left\|n^{m-1}\right\|_{H^{2}}^{2}\left\|\xi_{c}^{m-1}\right\|_{L^{2}}^{2} .
\end{aligned}
$$

Finally, the inductive hypothesis (6.1) can be verified in the same spirit of the two-dimensional case (see Sect. 5.2.2).

From decompositions (5.14)-(5.18), Theorem 6.1 and interpolation errors (3.3) and (3.6), one can deduce the following result:

Corollary 6.2. Under the assumptions of Theorem 6.1, the following estimates for the total errors hold

$$
\begin{aligned}
\left\|\left[e_{n}^{m}, e_{c}^{m}, e_{\mathbf{u}}^{m}, e_{\boldsymbol{\sigma}}^{m}\right]\right\|_{l^{\infty}\left(L^{2}\right)} & \leq C(T)\left(\Delta t+\max \left\{h^{r_{1}+1}, h^{r_{2}+1}, h^{r_{3}+1}, h^{r+1}\right\}\right), \\
\left\|\left[e_{n}^{m}, e_{c}^{m}, e_{\mathbf{u}}^{m}, e_{\boldsymbol{\sigma}}^{m}\right]\right\|_{l^{2}\left(H^{1}\right)} & \leq C(T)\left(\Delta t+\max \left\{h^{r_{1}}, h^{r_{2}}, h^{r_{3}}, h^{r}\right\}\right), \\
\left\|e_{\mathbf{u}}^{m}\right\|_{l^{\infty}\left(H^{1}\right)} & \leq C(T)\left(\Delta t+\max \left\{h^{r_{1}+1}, h^{r_{2}+1}, h^{r_{3}+1}, h^{r}\right\}\right),
\end{aligned}
$$

where the constant $C(T)>0$ is independent of $m, \Delta t$ and $h$. 


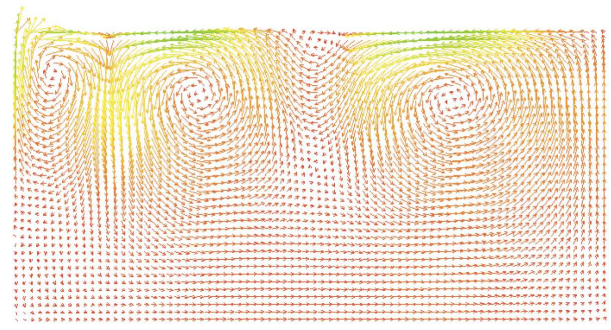

(a)

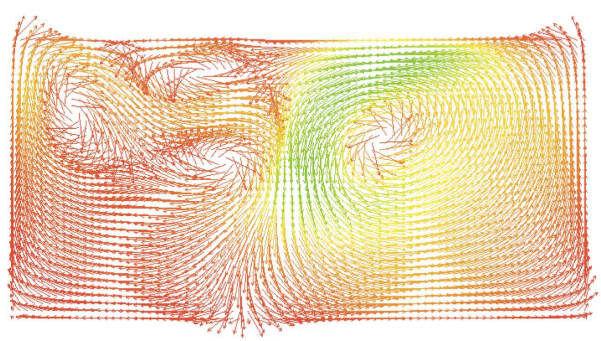

(c)

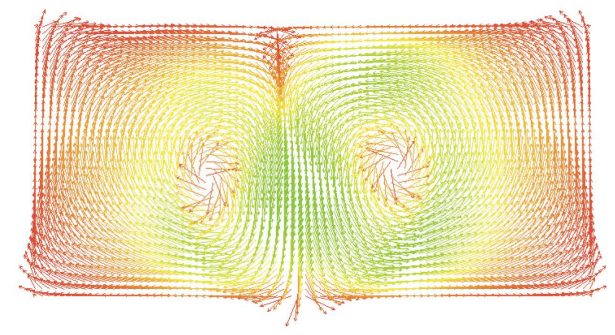

(e)

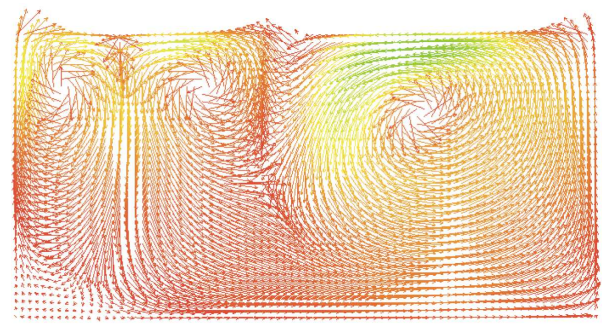

(b)

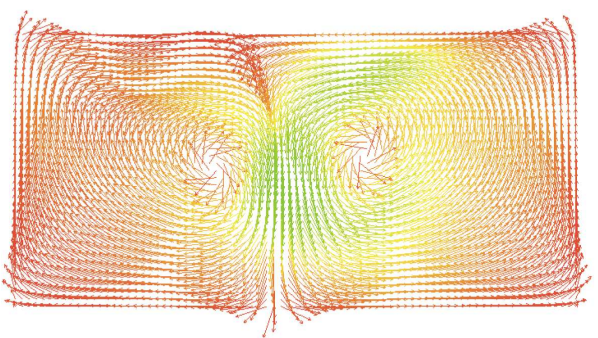

(d)

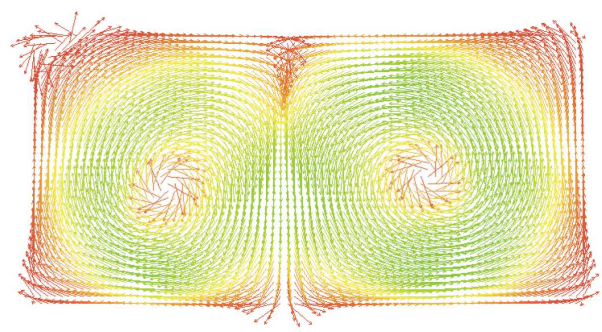

(f)

Figure 1. Evolution of the velocity field of the fluid. (a) $t=10^{-5}$; (b) $t=2 \times 10^{-4}$; (c) $t=5 \times 10^{-4}$; (d) $t=10^{-3}$; (e) $t=2 \times 10^{-3}$; and (f) $t=5 \times 10^{-3}$.

\section{Numerical Simulations}

In this section, we present two numerical experiments: the first one is used to verify that our scheme gives a good approximation to chemotaxis phenomena in a liquid environment; and the second has been considered in order to check numerically the error estimates proved in our theoretical analysis. All the numerical results are computed by using the software Freefem ++ . We have considered the spaces for $\eta, c, \boldsymbol{\sigma}, \mathbf{u}$ and $\pi$, generated by $\mathbb{P}_{1}, \mathbb{P}_{1}, \mathbb{P}_{1}, \mathbb{P}_{1}$ - bubble, $\mathbb{P}_{1}$-continuous FE, respectively.

Test 1 . In this experiment we consider the rectangular domain $\Omega=[0,2] \times[0,1]$, and the initial conditions

$$
\begin{aligned}
\eta_{0} & =\sum_{i=1}^{3}\left(70 \exp \left(-8\left(x-s_{i}\right)^{2}-10(y-1)^{2}\right)\right), \\
c_{0} & =30 \exp \left(-5(x-1)^{2}-5(y-0.5)^{2}\right) \\
\mathbf{u}_{0} & =\mathbf{0}
\end{aligned}
$$

where $s_{1}=0.2, s_{2}=0.5$ and $s_{3}=1.2$. The numerical solution is computed with mesh parameter $h=1 / 40$ and time step $\Delta t=10^{-5}$. Additionally, we consider the parameters values $\chi=8, D_{n}=4$, 


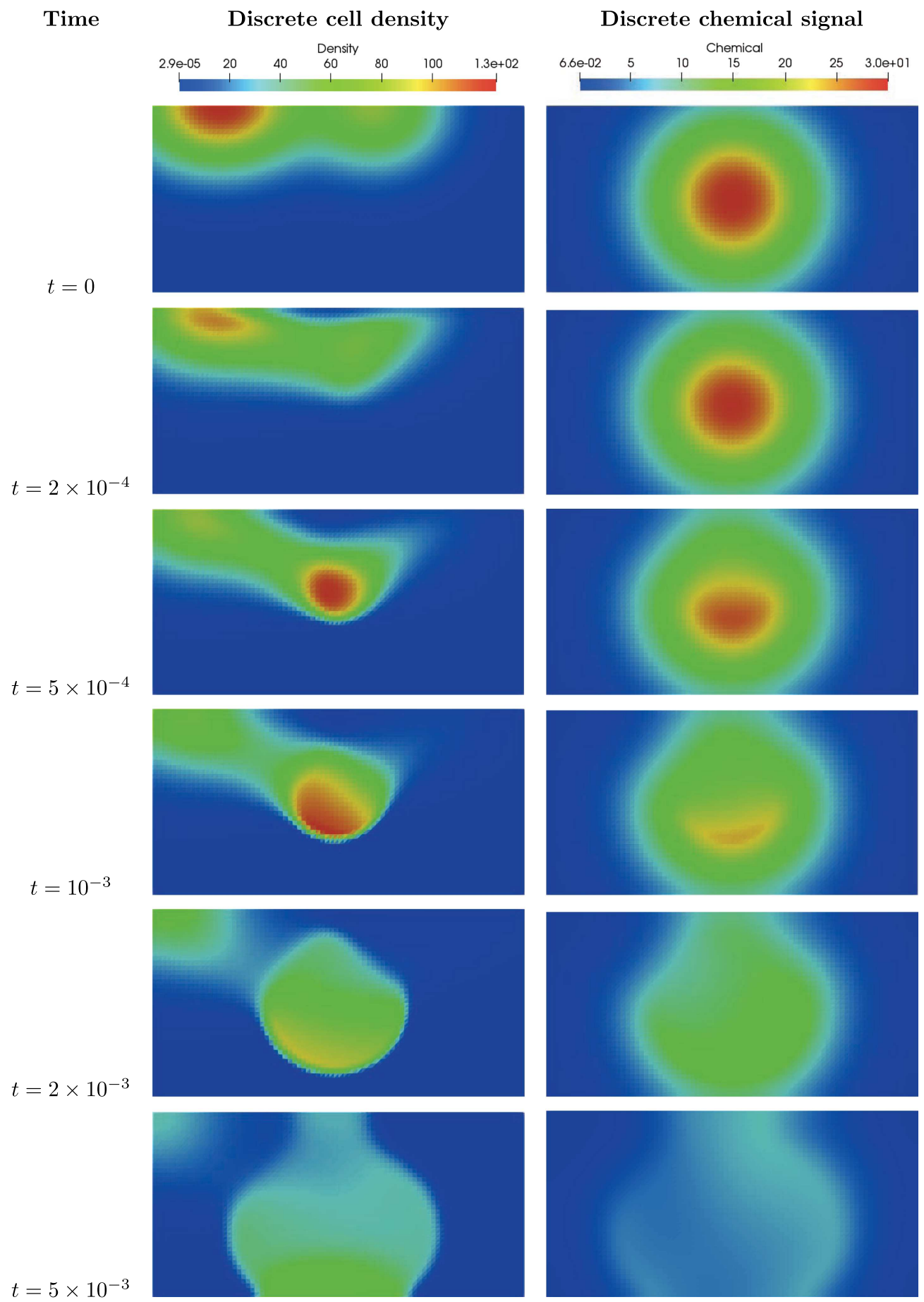

Figure 2. Cell density vs. chemical concentration. 
TABLE 1. Convergence rates in space for $\eta$.

\begin{tabular}{lllll}
\hline \hline$k \times k$ & $\left\|\eta\left(t_{m}\right)-\eta_{h}^{m}\right\|_{l^{\infty}\left(L^{2}\right)}$ & Order & $\left\|\eta\left(t_{m}\right)-\eta_{h}^{m}\right\|_{l^{2}\left(H^{1}\right)}$ & Order \\
\hline $10 \times 10$ & $5.7265 \times 10^{-2}$ & - & $1.1682 \times 10^{-1}$ & - \\
$20 \times 20$ & $1.4350 \times 10^{-2}$ & 1.9966 & $5.7520 \times 10^{-2}$ & 1.0223 \\
$30 \times 30$ & $6.3060 \times 10^{-3}$ & 2.0279 & $3.8242 \times 10^{-2}$ & 1.0067 \\
$40 \times 40$ & $3.4829 \times 10^{-3}$ & 2.0635 & $2.8656 \times 10^{-2}$ & 1.0031 \\
$50 \times 50$ & $2.1757 \times 10^{-3}$ & 2.1085 & $2.2915 \times 10^{-2}$ & 1.0017 \\
\hline
\end{tabular}

TABLE 2. Convergence rates in space for $c$.

\begin{tabular}{lllll}
\hline \hline$k \times k$ & $\left\|c\left(t_{m}\right)-c_{h}^{m}\right\|_{l^{\infty}\left(L^{2}\right)}$ & Order & $\left\|c\left(t_{m}\right)-c_{h}^{m}\right\|_{l^{2}\left(H^{1}\right)}$ & Order \\
\hline $10 \times 10$ & $3.5731 \times 10^{-2}$ & - & $1.1338 \times 10^{-1}$ & - \\
$20 \times 20$ & $8.9904 \times 10^{-3}$ & 1.9907 & $5.7106 \times 10^{-2}$ & 0.9895 \\
$30 \times 30$ & $4.0004 \times 10^{-3}$ & 1.9971 & $3.8126 \times 10^{-2}$ & 0.9964 \\
$40 \times 40$ & $2.2512 \times 10^{-3}$ & 1.9986 & $2.8610 \times 10^{-2}$ & 0.9981 \\
$50 \times 50$ & $1.4410 \times 10^{-3}$ & 1.9991 & $2.2894 \times 10^{-2}$ & 0.9989 \\
\hline
\end{tabular}

$\gamma=6, D_{\mathbf{u}}=10, D_{c}=\rho=1$ and $\phi(x, y)=-1000 y$. We show the simulations results for the times $t=0,2 \times 10^{-4}, 5 \times 10^{-4}, 10^{-3}, 2 \times 10^{-3}, 5 \times 10^{-3}$. The evolution of the velocity field is shown in Figure 1 , while the evolution results for the cell density and chemical signal are presented in Figure 2.

Initially, the cells are in two clusters in the upper part of our domain, and we observe that they begin to orient their movement in the direction of greater concentration of the chemical signal (in this case, the center of the domain). We can see how the clusters of organisms generate a kind of bridge between them and after, we see how organisms tend to agglomerate in the center of the rectangle. This interesting behavior occurs because the cross-diffusion term (or chemotaxis term) is the dominant one in the initial times. However, as time progresses, the chemical signal is consumed, which causes that the cross-diffusion loses strength, and the self-diffusion of the cells begins to dominate, and therefore they begin to distribute themselves homogeneously over the domain. It is also observed that, at the end, the cells move towards the bottom of the domain, which is due to the external force (since $\nabla \phi=(0,-1000)$ ). Finally, some changes in the velocity field are evidenced, influenced by the movement of the cells.

Test 2 (Convergence rates). In this experiment we take $\Omega=[0,1] \times[0,1]$ and we consider the exact solutions $\eta=$ $e^{-t}(\cos (2 \pi x)+\cos (2 \pi y)+3), c=e^{-t}(\sin (2 \pi y)+\cos (2 \pi x)-2 \pi y+9), \boldsymbol{\sigma}=\nabla c=2 \pi e^{-t}(-\sin (2 \pi x), \cos (2 \pi y)-1)$, $\mathbf{u}=e^{-t}(\sin (2 \pi y)(-\cos (2 \pi x+\pi)-1), \sin (2 \pi x)(\cos (2 \pi y+\pi)+1))$ and $\pi=e^{-t}(\cos (2 \pi x)+\sin (2 \pi y))$; and all parameters in (3.15) equal to 1 . Note that $\mathbf{u}=\mathbf{0}$ and $\frac{\partial c}{\partial \boldsymbol{\nu}}=\frac{\partial \eta}{\partial \boldsymbol{\nu}}=0$ on $\partial \Omega, \nabla \cdot \mathbf{u}=0$ in $\Omega$ and $\int_{\Omega} \pi=0$. Moreover, we use a uniform partition with $k+1$ nodes in each direction.

On the one hand, numerical results of spatial convergence rates are presented in Tables $1-5$ for $\Delta t=2 \times 10^{-4}$ with respect to time $T=0.01$. We observe the second-order convergence in space for the total errors in $e_{\eta}^{m}, e_{c}^{m}, e_{\mathbf{u}}^{m}$ in $l^{\infty}\left(L^{2}\right)$-norm, and the first-order convergence for $e_{\eta}^{m}, e_{c}^{m}, e_{\mathbf{u}}^{m}$ in $l^{2}\left(H^{1}\right)$-norm and $e_{\mathbf{u}}^{m}$ in $l^{\infty}\left(H^{1}\right)$-norm, which is in agreement with our theoretical analysis.

On the other hand, some numerical results of convergence rates in time are listed in Tables $6-8$ for $h=1 / 160$ (that is, $k=160$ nodes in space in each direction), with respect to time $T=5$. We observe the first-order convergence in time in weak and strong norms for all variables, which is in agreement with our theoretical analysis. 
TABLE 3. Convergence rates in space for $\mathbf{u}_{1}$ in weak norms.

\begin{tabular}{lllll}
\hline \hline$k \times k$ & $\left\|\mathbf{u}_{1}\left(t_{m}\right)-\left(\mathbf{u}_{1}\right)_{h}^{m}\right\|_{l^{\infty}\left(L^{2}\right)}$ & Order & $\left\|\mathbf{u}_{1}\left(t_{m}\right)-\left(\mathbf{u}_{1}\right)_{h}^{m}\right\|_{l^{2}\left(H^{1}\right)}$ & Order \\
\hline $10 \times 10$ & $4.1118 \times 10^{-2}$ & - & $1.5654 \times 10^{-1}$ & - \\
$20 \times 20$ & $1.0106 \times 10^{-2}$ & 2.0245 & $7.7874 \times 10^{-2}$ & 1.0074 \\
$30 \times 30$ & $4.4569 \times 10^{-3}$ & 2.0192 & $5.1820 \times 10^{-2}$ & 1.0045 \\
$40 \times 40$ & $2.4902 \times 10^{-3}$ & 2.0234 & $3.8827 \times 10^{-2}$ & 1.0034 \\
$50 \times 50$ & $1.5822 \times 10^{-3}$ & 2.0324 & $3.1043 \times 10^{-2}$ & 1.0027 \\
\hline
\end{tabular}

TABLE 4. Convergence rates in space for $\mathbf{u}_{2}$ in weak norms.

\begin{tabular}{lllll}
\hline \hline$k \times k$ & $\left\|\mathbf{u}_{2}\left(t_{m}\right)-\left(\mathbf{u}_{2}\right)_{h}^{m}\right\|_{l^{\infty}\left(L^{2}\right)}$ & Order & $\left\|\mathbf{u}_{2}\left(t_{m}\right)-\left(\mathbf{u}_{2}\right)_{h}^{m}\right\|_{l^{2}\left(H^{1}\right)}$ & Order \\
\hline $10 \times 10$ & $4.1175 \times 10^{-2}$ & - & $1.5655 \times 10^{-1}$ & - \\
$20 \times 20$ & $1.0125 \times 10^{-2}$ & 2.0238 & $7.7875 \times 10^{-2}$ & 1.0075 \\
$30 \times 30$ & $4.4658 \times 10^{-3}$ & 2.0190 & $5.1821 \times 10^{-2}$ & 1.0046 \\
$40 \times 40$ & $2.4952 \times 10^{-3}$ & 2.0232 & $3.8827 \times 10^{-2}$ & 1.0034 \\
$50 \times 50$ & $1.5855 \times 10^{-3}$ & 2.0322 & $3.1043 \times 10^{-2}$ & 1.0027 \\
\hline
\end{tabular}

TABLE 5. Convergence rates in space for $\mathbf{u}_{1}$ and $\mathbf{u}_{2}$ in strong norms.

\begin{tabular}{lllll}
\hline \hline$k \times k$ & $\left\|\mathbf{u}_{1}\left(t_{m}\right)-\left(\mathbf{u}_{1}\right)_{h}^{m}\right\|_{l^{\infty}\left(H^{1}\right)}$ & Order & $\left\|\mathbf{u}_{2}\left(t_{m}\right)-\left(\mathbf{u}_{2}\right)_{h}^{m}\right\|_{l^{\infty}\left(H^{1}\right)}$ & Order \\
\hline $10 \times 10$ & 2.3353 & - & 2.3353 & - \\
$20 \times 20$ & 1.1882 & 0.9747 & 1.1882 & 0.9747 \\
$30 \times 30$ & $7.9477 \times 10^{-1}$ & 0.9920 & $7.9477 \times 10^{-1}$ & 0.9920 \\
$40 \times 40$ & $5.9675 \times 10^{-1}$ & 0.9960 & $5.9675 \times 10^{-1}$ & 0.9960 \\
$50 \times 50$ & $4.7765 \times 10^{-1}$ & 0.9976 & $4.7765 \times 10^{-1}$ & 0.9976 \\
\hline
\end{tabular}

TABLE 6. Convergence rates in time for $\eta$ and $c$.

\begin{tabular}{lllll}
\hline \hline$\Delta t$ & $\left\|\eta\left(t_{m}\right)-\eta_{h}^{m}\right\|_{l^{\infty}\left(L^{2}\right)}$ & Order & $\left\|c\left(t_{m}\right)-c_{h}^{m}\right\|_{l^{2}\left(H^{1}\right)}$ & Order \\
\hline $1.56 \times 10^{-1}$ & $7.6767 \times 10^{-1}$ & - & 1.1401 & - \\
$1.25 \times 10^{-1}$ & $6.1364 \times 10^{-1}$ & 1.0036 & $8.9916 \times 10^{-1}$ & 1.0642 \\
$1.04 \times 10^{-1}$ & $5.0302 \times 10^{-1}$ & 1.0902 & $7.4178 \times 10^{-1}$ & 1.0553 \\
$8.93 \times 10^{-2}$ & $4.2089 \times 10^{-1}$ & 1.1564 & $6.3126 \times 10^{-1}$ & 1.0466 \\
\hline
\end{tabular}

TABLE 7. Convergence rates in time for $\mathbf{u}_{1}$ and $\mathbf{u}_{2}$ in weak norms.

\begin{tabular}{lllll}
\hline \hline$\Delta t$ & $\left\|\mathbf{u}_{1}\left(t_{m}\right)-\left(\mathbf{u}_{1}\right)_{h}^{m}\right\|_{l^{\infty}\left(L^{2}\right)}$ & Order & $\left\|\mathbf{u}_{2}\left(t_{m}\right)-\left(\mathbf{u}_{2}\right)_{h}^{m}\right\|_{l^{2}\left(H^{1}\right)}$ & Order \\
\hline $1.56 \times 10^{-1}$ & $7.5647 \times 10^{-2}$ & - & $4.0781 \times 10^{-1}$ & - \\
$1.25 \times 10^{-1}$ & $6.0890 \times 10^{-2}$ & 0.9724 & $3.2581 \times 10^{-1}$ & 1.0060 \\
$1.04 \times 10^{-1}$ & $5.0548 \times 10^{-2}$ & 1.0210 & $2.7240 \times 10^{-1}$ & 0.9820 \\
$8.93 \times 10^{-2}$ & $4.2940 \times 10^{-2}$ & 1.0582 & $2.3520 \times 10^{-1}$ & 0.9526 \\
\hline
\end{tabular}


TABLE 8. Convergence rates in time for $\mathbf{u}_{1}$ and $\mathbf{u}_{2}$ in strong norms.

\begin{tabular}{lllll}
\hline \hline$\Delta t$ & $\left\|\mathbf{u}_{1}\left(t_{m}\right)-\left(\mathbf{u}_{1}\right)_{h}^{m}\right\|_{l^{\infty}\left(H^{1}\right)}$ & Order & $\left\|\mathbf{u}_{2}\left(t_{m}\right)-\left(\mathbf{u}_{2}\right)_{h}^{m}\right\|_{l^{\infty}\left(H^{1}\right)}$ & Order \\
\hline $1.56 \times 10^{-1}$ & $5.6495 \times 10^{-1}$ & - & $5.8574 \times 10^{-1}$ & - \\
$1.25 \times 10^{-1}$ & $4.5726 \times 10^{-1}$ & 0.9477 & $4.7259 \times 10^{-1}$ & 0.9619 \\
$1.04 \times 10^{-1}$ & $3.8249 \times 10^{-1}$ & 0.9793 & $3.9429 \times 10^{-1}$ & 0.9936 \\
$8.93 \times 10^{-2}$ & $3.2809 \times 10^{-1}$ & 0.9952 & $3.3744 \times 10^{-1}$ & 1.0100 \\
\hline
\end{tabular}

Acknowledgements. The third and fourth authors have been supported by Vicerrectoría de Investigación y Extensión of Universidad Industrial de Santander, Capital Semilla Project, code 2491. The second author has been partially supported by PGC2018-098308-B-I00 (MCI/AEI/FEDER, UE). The authors would like to thank the anonymous referees for their valuable comments.

\section{REFERENCES}

[1] C. Amrouche and N.E.H. Seloula, $L^{p}$-theory for vector potentials and Sobolev's inequalities for vector fields: application to the Stokes equations with pressure boundary conditions. Math. Models Methods Appl. Sci. 23 (2013) 37-92.

[2] M. Bessemoulin-Chatard and A. Jüngel, A finite volume scheme for a Keller-Segel model with additional cross-diffusion. IMA J. Numer. Anal. 34 (2014) 96-122.

[3] G. Chamoun, M. Saad and R. Talhouk, Numerical analysis of a chemotaxis-swimming bacteria model on a general triangular mesh. Appl. Numer. Math. 127 (2018) 324-348.

[4] A. Chertock, K. Fellner, A. Kurganov, A. Lorz and P. Markowich, Sinking, merging and stationary plumes in a coupled chemotaxis-fluid model: a high-resolution numerical approach. J. Fluid Mech. 694 (2012) 155-190.

[5] M. Cui, Analysis of a semidiscrete discontinuous Galerkin scheme for compressible miscible displacement problem. J. Comput. Appl. Math. 214 (2008) 617-636.

[6] Y. Deleuze, C.-Y. Chiang, M. Thiriet and T.W. Sheu, Numerical study of plume patterns in a chemotaxis-diffusion-convection coupling system. Comput. Fluids 126 (2016) 58-70.

[7] J. Douglas, Jr. and J.E. Roberts, Numerical methods for a model for compressible miscible displacement in porous media. Math. Comput. 41 (1983) 441-459.

[8] A. Duarte-Rodríguez, L.C.F. Ferreira and E.J. Villamizar-Roa, Global existence for an attraction-repulsion chemotaxis fluid model with logistic source. Disc. Contin. Dyn. Syst. Ser. B 24 (2019) 423-447.

[9] Y. Epshteyn and A. Izmirlioglu, Fully discrete analysis of a discontinuous finite element method for the Keller-Segel chemotaxis model. J. Sci. Comput. 40 (2009) 211-256.

[10] F. Filbet, A finite volume scheme for the Patlak-Keller-Segel chemotaxis model. Numer. Math. 104 (2006) $457-488$.

[11] V. Girault and P.-A. Raviart, Finite Element Methods for Navier-Stokes Equations. Theory and Algorithms. Springer-Verlag (1986).

[12] F. Guillén-González and M.V. Redondo-Neble, Spatial error estimates for a finite element viscosity-splitting scheme for the Navier-Stokes equations. Int. J. Numer. Anal. Model. 10 (2013) 826-844.

[13] F. Guillén-González, M.A. Rodríguez-Bellido and D.A. Rueda-Gómez, Unconditionally energy stable fully discrete schemes for a chemo-repulsion model. Math. Comput. 88 (2019) 2069-2099.

[14] F. Guillén-González, M.A. Rodríguez-Bellido and D.A. Rueda-Gómez, Study of a chemo-repulsion model with quadratic production. Part I: analysis of the continuous problem and time-discrete numerical schemes. Comput. Math. App. 80 (2020) 692-713.

[15] F. Guillén-González, M.A. Rodríguez-Bellido and D.A. Rueda-Gómez, Study of a chemo-repulsion model with quadratic production. Part II: analysis of an unconditionally energy-stable fully discrete scheme. Comput. Math. App. 80 (2020) 636652.

[16] J. Heywood and R. Rannacher, Finite element approximation of the nonstationary Navier-Stokes problem. IV. Error analysis for second order time discretization. SIAM J. Numer. Anal. 27 (1990) 353-384.

[17] N. Hill and T. Pedley, Bioconvection. Fluid Dyn. Res. 37 (2005) 1-20.

[18] J. Jiang, H. Wu and S. Zheng, Global existence and asymptotic behavior of solutions to a chemotaxis-fluid system on general bounded domains. Asympt. Anal. 92 (2015) 249-258.

[19] A. Karimi and M. Paul, Bioconvection in spatially extended domains. Phys. Rev. E 87 (2013) 53016.

[20] J. Lankeit, Long-term behaviour in a chemotaxis-fluid system with logistic source. Math. Models Methods Appl. Sci. 26 (2016) 2071-2109.

[21] H.G. Lee and J. Kim, Numerical investigation of falling bacterial plumes caused by bioconvection in a three-dimensional chamber. Eur. J. Mech. B/Fluids 52 (2015) 120-130. 
[22] A. Lorz, Coupled Keller-Segel Stokes model: global existence for small initial data and blow-up delay. Commun. Math. Sci. 10 (2012) 555-574.

[23] A. Marrocco, Numerical simulation of chemotactic bacteria aggregation via mixed finite elements. ESAIM: M2AN 37 (2003) $617-630$.

[24] N. Mizoguchi and P. Souplet, Nondegeneracy of blow-up points for the parabolic Keller-Segel system. Ann. Inst. Henri Poincaré Non Linéaire Anal. 31 (2014) 851-875.

[25] J. Necas, Les Méthodes Directes en Théorie des Equations Elliptiques. Editeurs Academia, Prague (1967).

[26] N. Saito, Conservative upwind finite-element method for a simplified Keller-Segel system modelling chemotaxis. IMA J. Numer. Anal. 27 (2007) 332-365.

[27] N. Saito, Error analysis of a conservative finite-element approximation for the Keller-Segel system of chemotaxis. Commun. Pure Appl. Anal. 11 (2012) 339-364.

[28] R. Stenberg, A technique for analysing finite element methods for viscous incompressible flow. Int. J. Numer. Methods Fluids 11 (1990) 935-948.

[29] Y. Tao and M. Winkler, Boundedness and decay enforced by quadratic degradation in a three-dimensional chemotaxis-fluid system. Z. Angew. Math. Phys. 66 (2015) 2555-2573.

[30] I. Tuval, L. Cisneros, C. Dombrowski, C.W. Wolgemuth, J.O. Kessler and R.E. Goldstein, Bacterial swimming and oxygen transport near contact lines. Proc. Nat. Acad. Sci. USA 102 (2005) 2277-2282.

[31] M. Winkler, Global large-data solutions in a chemotaxis-(Navier-)Stokes system modelling cellular swimming in fluid drops. Commun. Part. Differ. Equ. 37 (2012) 319-351.

[32] M. Winkler, Stabilization in a two-dimensional chemotaxis-Navier-Stokes system. Arch. Ration. Mech. Anal. 211 (2014) $455-487$.

[33] M. Winkler, Global weak solutions in a three-dimensional chemotaxis-Navier-Stokes system. Ann. Inst. Henri Poincaré (C) Non Linear Anal. 33 (2016) 1329-1352.

[34] J. Yang and Y. Chen, Superconvergence of a combined mixed finite element and discontinuous Galerkin method for a compressible miscible displacement problem. Acta Math. Appl. Sin. Eng. Ser. 27 (2011) 481-494.

[35] Q. Zhang and Y. Li, Convergence rates of solutions for a two-dimensional chemotaxis-Navier-Stokes system. Disc. Contin. Dyn. Syst. Ser. B $\mathbf{2 0}(2015)$ 2751-2759.

[36] J. Zhang, J. Zhu and R. Zhang, Characteristic splitting mixed finite element analysis of Keller-Segel chemotaxis models. Appl. Math. Comput. 278 (2016) 33-44. 